University of Louisville

ThinkIR: The University of Louisville's Institutional Repository

8-2014

\title{
Evaluation of the safety and pharmacokinetic profile of the broad spectrum antiviral lectin Griffithsin.
}

Christopher Lynn Barton

University of Louisville

Follow this and additional works at: https://ir.library.louisville.edu/etd

Part of the Pharmacy and Pharmaceutical Sciences Commons

\section{Recommended Citation}

Barton, Christopher Lynn, "Evaluation of the safety and pharmacokinetic profile of the broad spectrum antiviral lectin Griffithsin." (2014). Electronic Theses and Dissertations. Paper 80.

https://doi.org/10.18297/etd/80

This Doctoral Dissertation is brought to you for free and open access by ThinkIR: The University of Louisville's Institutional Repository. It has been accepted for inclusion in Electronic Theses and Dissertations by an authorized administrator of ThinkIR: The University of Louisville's Institutional Repository. This title appears here courtesy of the author, who has retained all other copyrights. For more information, please contact thinkir@louisville.edu. 


\title{
EVALUATION OF THE SAFETY AND PHARMACOKINETIC PROFILE OF THE BROAD SPECTRUM ANTIVIRAL LECTIN GRIFFITHSIN
}

\author{
By \\ Christopher Lynn Barton \\ B.A., University of Louisville, 1998 \\ J.D., University of Louisville, 2002 \\ M.S., University of Louisville, 2011

\begin{abstract}
A Dissertation
Submitted to the Faculty of the

The University of Louisville School of Medicine

In Partial Fulfillment of the Requirements

For the Degree of
\end{abstract} \\ Doctor of Philosophy \\ Department of Pharmacology and Toxicology \\ University of Louisville \\ Louisville, Kentucky
}

August 2014 

EVALUATION OF THE SAFETY AND PHARMACOKINETIC PROFILE OF THE BROAD SPECTRUM ANTIVIRAL LECTIN GRIFFITHSIN

By

Christopher Lynn Barton

B.A., University of Louisville, 1998

J.D., University of Louisville, 2002

M.S., University of Louisville, 2011

A Dissertation Approved on

June 23, 2014

By the following Dissertation Committee:

Kenneth E. Palmer, Ph.D.

J. Brad Chaires, Ph.D.

Theresa Chen, Ph.D.

Harrell Hurst, Ph.D.

Nobuyuki Matoba, Ph.D. 


\section{DEDICATION}

This Dissertation is dedicated to my partner

Christopher L. Dennison and niece, Crystal Barton

who have sojourned aside me through my years of study. 


\section{ACKNOWLEDGMENTS}

I would like to thank my mentor, Dr. Kenneth Palmer, for his guidance and support. I would also like to thank my other committee members, Drs. Nobi Matoba, Theresa Chen, Harrell Hurst, and Brad Chaires for providing insight into my projects. Finally I would like to thank Amanda Lasnik, Calvin Kouokam, Nichola Garbett, Keegan Baldauf, Tiffany Grooms-Williams, Adam Husk, Sarah Wilcher and the late Janice Ditslear for passing on their technical knowledge and contributing to the experiments described herein. 


\begin{abstract}
EVALUATION OF THE SAFETY AND PHARMACOKINETIC PROFILE OF THE BROAD SPECTRUM ANTIVIRAL LECTIN GRIFFITHSIN

Christopher Lynn Barton

June 23, 2014
\end{abstract}

Carbohydrate binding agents that target viral envelope glycans are being studied for their potential use as microbicides and antiviral therapeutics. Griffithsin is a lectin originally identified in a red alga Griffithisia sp. Multiple studies have shown that GRFT inhibits HIV-1, Coronaviruses, Hepatitis C, influenza and Ebola virus replication in vitro. This antiviral activity suggests potential uses in chemoprophylaxis and disease treatment. However, safety of GRFT administration has not been extensively studied. In vivo testing--chronic subcutaneous treatment as well as single dose subcutaneous, oral, and intravenous administrations of Griffithsin in Sprague Dawley Rats (Rattus Norvegicus)--was used to assess Griffithsin's pharmacokinetic properties and to predict whether use of Griffithsin for antiviral treatment might be safe and effective. Based on histological, serological, and biochemical data derived from these experiments, Griffithsin is generally well tolerated. However, protein 
binding assays revealed interactions with complement and apolipoproteins and calorimetric assays revealed changes in serum thermograms that may require further study. 
TABLE OF CONTENTS

PAGE

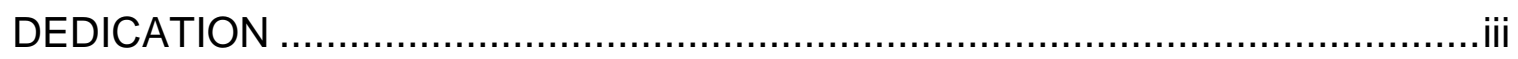

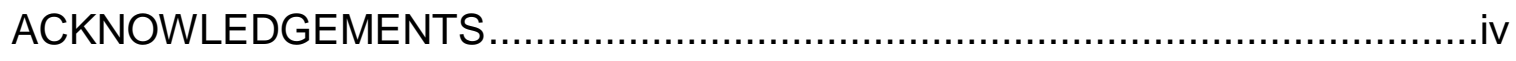

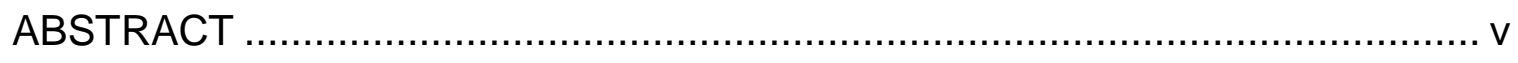

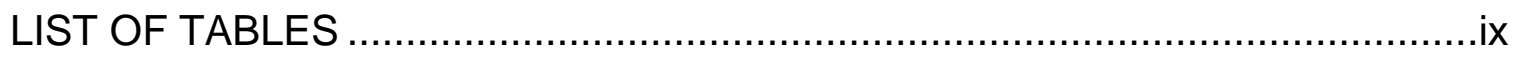

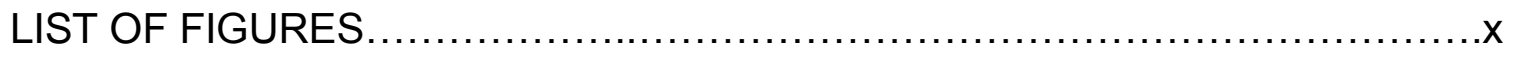

CHAPTER 1: INTRODUCTION AND BACKGROUND ................................... 1

CHAPTER 2: ACTIVITY AND EFFECT OF SUBCUTANEOUS TREATEMENT WITH THE BROAD SPECTRUM ANTIVIRAL LECTIN GRIFFITHSIN IN TWO

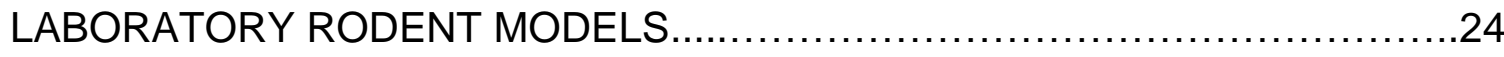

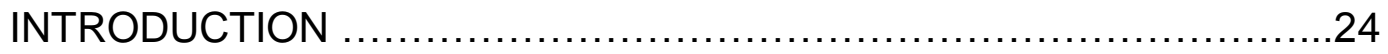

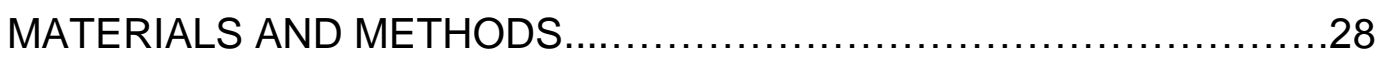

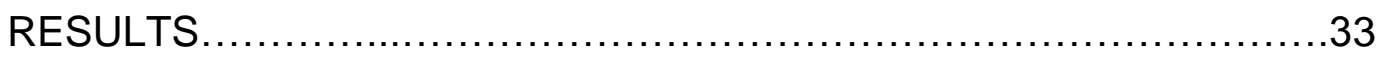

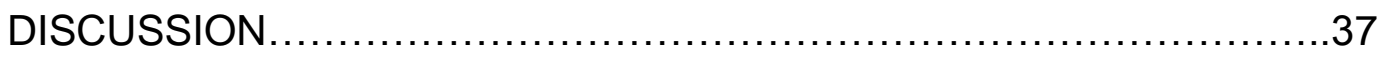

CHAPTER 3: THE PHARMACOKINETICS OF THE ANTIVIRAL LECTIN GRIFFITHSIN INDICATES MULTIPLE POTENTIAL USES AND

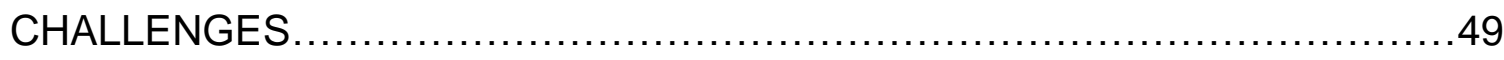

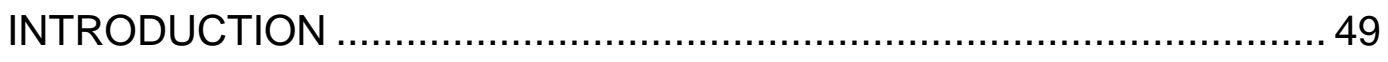

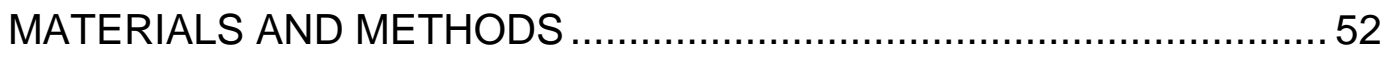

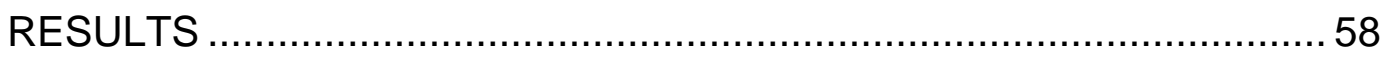


CHAPTER 4: SUB-CHRONIC SUBCUTANEOUS TREATMENT WITH THE ANTIVIRAL LECTIN GRIFFITHSIN IN RATS TO DETERMINE GRIFFITHSIN'S SYSTEMIC TOLERABILITY AND EXAMINE GRIFFITHSIN'S EFFECTS UPON ORGANS, BLOOD CELL COUNTS, SERUM CHEMISTRIES, AND CYTOKINE PRODUCTION

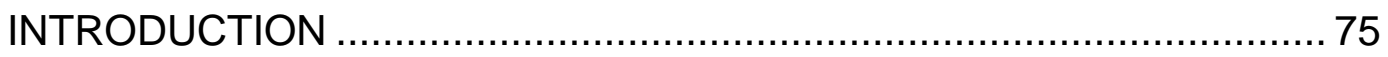

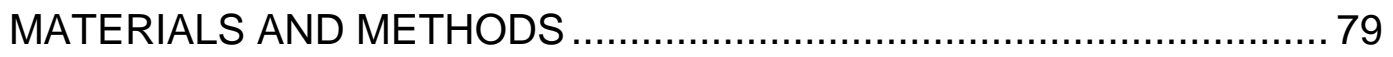

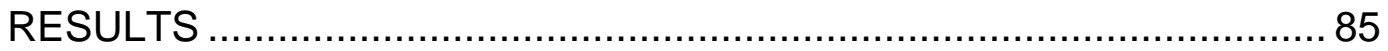

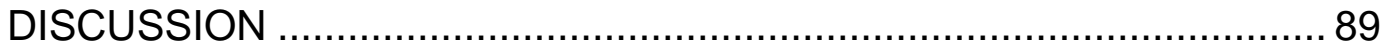

CHAPTER 5: CHARACTERIZATION OF BIOCHEMICAL INTERACTIONS OF GRIFFITHSIN THROUGH DIFFERENTIAL SCANNING FLUORIMETRY, AFFINITY PURIFICATION, AND DIFFERENTIAL SCANNING CALORIMETRY

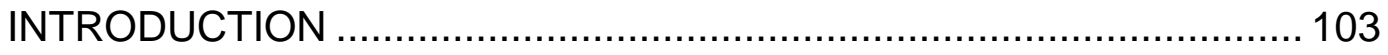

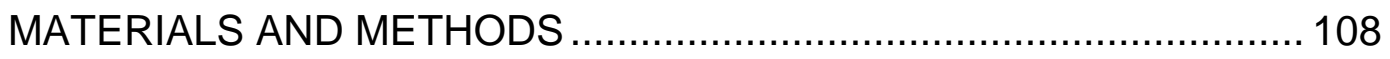

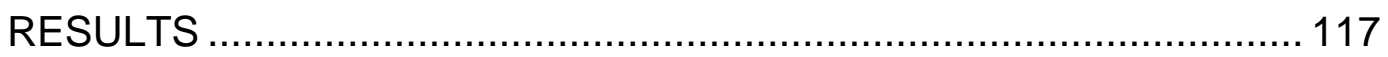

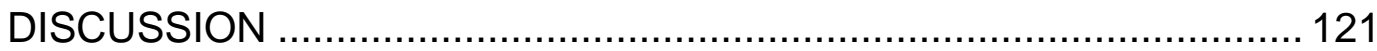

CHAPTER 6: SUMMARY OF GRIFFITHSIN RESEARCH AND IMPLICATIONS

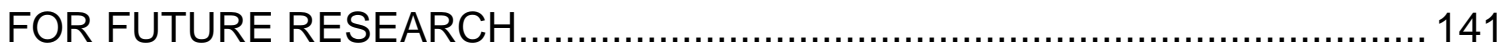

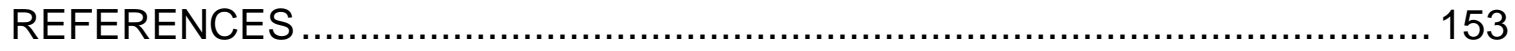

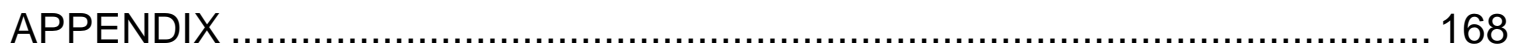

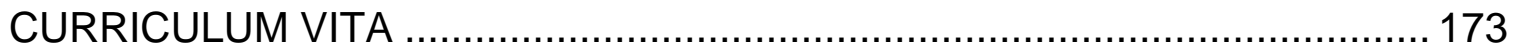




\section{LIST OF TABLES}

TABLE

PAGE

2.1 Hematological profile for guinea pigs after chronic treatment with GRFT 46

3.1 Pharmacokinetic Parameters of GRFT .................................71

4.1 Hematological profile for rats after chronic treatment with GRFT ........100

4.2 Serum Chemistry panels of animals after chronic treatment

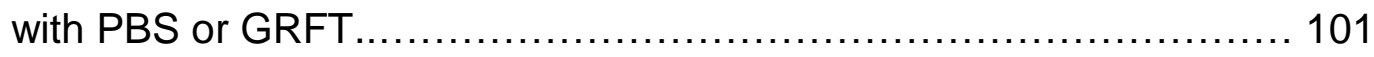

4.3 Cytokine levels after chronic administration with GRFT ................102

5.1 Concentration dependent shifts in melting temperatures of GRFT and GRFT $^{\text {lec- }}$ determined by SYPRO orange fluorescence shifts

5.2 Proteins recovered from Human Serum by Affinity Purification with FLAGfusion protein 132

5.3 Unique Proteins identified by affinity purification 133

5.4 Pearson Correlations of rat serum thermogram parameters and rat serum characteristics 140 


\section{LIST OF FIGURES}

FIGURE

PAGE

1.1 Figure 1.1 N-linked Glycans - structural arrangement of subtypes .......23

2.1 Griffithsin concentrations in plasma and serum samples ................ 41

2.2 Antiviral activity of samples collected from GRFT treated animals ........42

2.3 Quantitation of GRFT in mouse organs ................................43

2.4 Guinea pig body weight gain as an indicator of overall health.............44

2.5 GRFT does not affect guinea pig organ weights........................45

2.6 Serum chemistry data for guinea pigs after chronic treatment

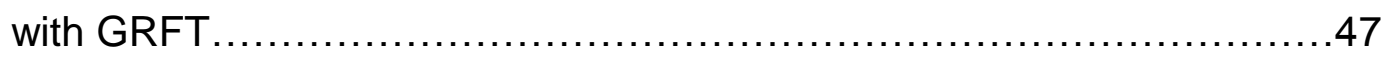

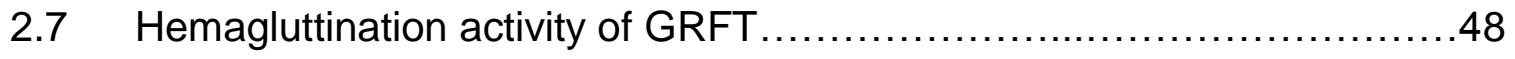

3.1 Pharmacokinetic profile of GRFT in Serum .............................70

3.2 GRFT Concentrations in Fecal Extracts following chronic oral dosing .....72

3.3 Antiviral activity of pooled fecal extracts collected on select days during the course of chronic oral treatment with GRFT. 73

3.4 Total GRFT recovery following single administration $\ldots \ldots \ldots \ldots \ldots \ldots \ldots . \ldots \ldots$

4.1 Treatment Regimen to evaluate the impact of an immune response upon GRFT's systemic tolerance and activity .96

4.2 GRFT serum concentrations in rats during and after chronic GRFT treatment and comparisons with single dose pharmacokinetic 
values

4.3 Titers of anti-GRFT IgG and antiviral activity of sera from rats preimmunized against GRFT, passively immunized with GRFT IgG, and controls .98

4.4 Starting weight, weight gain over treatment, and Organ percentages from rats preimmunized against GRFT, passively immunized with GRFT IgG, and controls .99

5.1 GRFT and GRFT ${ }^{\text {lec- }}$ SYPRO orange denaturation curves in the absence of saccharide ligand 129

5.2 Protein stabilization effects of ligand binding on GRFT and GRFT ${ }^{\text {lec }} \ldots .130$

5.3 Thermogram profiles of rat serum spiked with varying concentrations of GRFT

5.4 Mean thermograms generated from sera from rats chronically treated with Griffithsin or PBS. 135

5.5 Thermogram shape and feature parameters for rats chronically treated with GRFT or PBS 136

5.6 Peak and First moment thermogram parameters for rats chronically treated with GRFT or PBS

5.7 Distribution of Excess specific heat capacity for rats chronically treated with GRFT or PBS at select temperature points 138

5.8 Thermogram similarity scores for sera from rats chronically treated with PBS or GRFT 


\section{CHAPTER 1}

\section{INTRODUCTION AND BACKGROUND}

A number of enveloped viruses continue present significant threats to public health in the United States and the developing world. Discovery and development of antivirals with broad spectrum activity is important for both antiviral prophylaxis as well as for therapy.

Perhaps the most significant viral epidemic in the recent past is Human Immunodeficiency Virus (HIV). With no effective vaccine, HIV-1, affects over 35 million people worldwide, and still claims the lives of millions of people per year despite effective treatment options [1]. In the United States alone, approximately 50,000 new infections still occur yearly, with over $50 \%$ of those infections being among men who have sex with men (MSM). Other enveloped viruses also cause persistent viral infections and present serious threats to public health. Aside from HIV-1, the most clinically significant persistent viral infections are caused by Hepatitis C Virus (HCV), and human herpesviruses such as cytomegalovirus (CMV), Karposi's sarcoma-associated herpesvirus (KSHV), and type 2 herpes simplex virus (HSV-2). Viruses that cause chronic, often life-long infections have 
all needed to evolve strategies to evade the host immune response. Viruses use a diverse range of immune evasion strategies, from blocking the interferon response through induction of interfering ribonucleic acids (RNAs) [2-7]. For example, HIV-1 employs many of these strategies ranging from inhibition of humoral immune responses by complement to antigenic variation attributable to low fidelity reverse transcriptase $[8,9]$. Further, HIV-1 downregulates interferon (IFN) production to prevent an immune response [10], as well as possibly encodes silencing RNAs [11, 12]. Many of the viruses that persist for long periods of time display dense glycan shields on their envelopes to evade humoral immune responses by display of "self" epitopes that occlude functionally important domains of the envelope proteins, and in some cases to suppress induction of an immune response.

Enveloped viruses from taxonomic families not necessarily linked by a common evolutionary history often display heavily glycosylated envelope glycoproteins. Dense, asparagine-linked (N-linked) glycans attached to viral surface proteins are a common structure amongst these viruses $[13,14]$. These glycans present on viral envelope proteins have roles in host immune evasion and modulation, as well as stabilization of viral envelope structures [15-17]. In the case of HIV, oligomannose glycans have been theorized to assist in immune evasion through presentation "self" epitopes [18], as well as down-regulation of INF production and immunosuppressive cytokine induction under some circumstances $[2,10,19,20]$. 
While glycans are structurally important for these viruses, they also present new targets for antiviral agents. Agents which could selectively bind these glycans could thus be attractive targets for ongoing antiviral research both as a preventative as well as a treatment [21]. In recent years, natural product derived lectins have been recognized for this very purpose [14, 22-24]. These lectins have been observed to inhibit viral transmission by binding to the specific N-linked glycans present on the surface of the viral envelopes [14]. In so doing they have the effect of preventing viral fusion and entry [22, 25-27].

Outcomes of lectin therapy have also generally been positive. Animal survival and amelioration of viral infection has been observed in a number of viral challenges. Treatment with cyanovirin (CV-N) conveyed marked survival benefits in a murine influenza challenge [28]. Treatment with large concentrations of human mannose binding lectin (MBL) conveyed a survival benefit versus Ebola virus challenge in mice [29]. Additionally, HCV infection has been modestly inhibited through lectin therapy [30, 31].

Griffithsin (GRFT) is a lectin originally identified from the red algae Griffithisae sp [32]. Now recombinantly produced in tobacco plants, GRFT is a candidate topical microbicide and systemic therapeutic [33, 34]. In vitro studies into GRFT's anti-HIV-1 activity have demonstrated an average inhibitory $\mathrm{EC}_{50}$ of $40 \mathrm{pM}$ [32]. While initially investigated as a microbicide, systemic administration studies are currently underway to evaluate potential uses of GRFT in antiviral therapy [34]. 
Given GRFT's potent antiviral activity and low immunogenicity, GRFT has the diverse potential applications, including use as a systemic anti-viral therapy, chemoprophylaxis, or microbicidal agent [35-37]. The purpose of this dissertation is to explore GRFT's potential usage as a pre-exposure prophylaxis and systemic therapeutic for enveloped viruses possessing high-mannose $\mathrm{N}$ linked glycans. While GRFT has activity against multiple viruses, this dissertation focuses on GRFT in the context of HIV-1 infection given robust in vitro assays to measure and analyze GRFT's anti-HIV activity in serum and other biological products. The specific aims of these studies included: evaluation of GRFT's practical pharmacokinetic parameters to elucidate how method of administration may impact GRFT's utility; evaluation of GRFT's potential as an orally dosed rectal microbicide through gastric lavage of different concentrations of GRFT in rats; evaluation of GRFT's systemic impacts both in the presence and absence of an antibody response with accompanying intermediate-term dosing; and identification of GRFT's potential endogenous serum protein binding partners through affinity purification; as well as evaluation as to whether Differential Scanning Calorimetry is capable of revealing alterations in serum protein interactions following both chronic systemic administration and serum spiking of GRFT.

\section{Glycosylation States}

Glycosylation is enzymatic addition of complex carbohydrate chains to organic molecules, such as proteins [38]. These additions assist in maintaining proper protein folding, increase protein stability, and contribute to protein 
functionality [39]. Furthermore, glycosylation can result in greater hydrophilicity. Viral glycosylation can also alter infectivity and viral fitness [40].

Glycosylation is a covalent post-translational modification that can occur in either the Golgi apparatus or the endoplasmic reticulum and is fundamental to many biological processes [29]. This modification process can result in five different types of glycans (glycoforms) can result: N-linked, O-linked, C-linked, glipiated, or phospho-glycans [29]. The glycoform relevant to the studies at hand are of the N-linked variety, given GRFT's high affinity for oligomannose N-linked glycans.

$\mathrm{N}$-linked glycosylation begins with the addition of a 14 unit oligosaccharide precursor to an asparagine in a polypeptide chain. The signal for $\mathrm{N}$-linked glycosylation occurs through one of two sequences: Asn-X-Ser or Asn-X-Thr, where $X$ can be any amino acid except proline [41]. The general branched carbohydrate chain contains 3 glucose, 9 mannose, and $2 \mathrm{~N}$-acetylglucosamine molecules attached to a carrier molecule (a dolichol), and transferred to the appropriate signal sequence on the protein as it is translocated into the ER lumen [42].

Saccharide compositions between glycans of the same linkage type may also vary, leading to High-mannose, Hybrid, or Complex glycans (with the terminal structure presented after editing determining the glycan subtype) (Figure 1.1) [29]. High-mannose glycans exclusively present mannose on the terminal portions of the sugar, and contain 5-9 mannose residues [29] (Figure 1.1 A \&B). No N-acetyl glucosamine residues are presented. As the initial state for many 
glycans, further editing occurs which result in either Complex (Figure 1.1 D) or Hybrid glycans (Figure 1.1C) [29]. This editing is thought to depend on accessibility to multiple saccharide-modifying proteins present in the Golgi [29]. Further editing results in either Hybrid glycans - where at least one mannose residue and one $\mathrm{N}$-acetylglucosamine are presented, or Complex glycans [29]. Complex glycans, which do not have mannose terminal residues, can have multiple $\mathrm{N}$-acetyl glucosamines or other terminal sugar structures presented [29].

Endogenous glycans have many vital physiological roles. Glycans can prevent self-recognition and autoimmunity[43]. Functionally, glycans can stabilize protein folds as well as become vital parts of extracellular matrix [44]. Further, glycans can function in cell trafficking and communication through recognition of glycan binding proteins[45]. In the case of antibodies and other circulating proteins, serum half-life and cell receptor interaction may be influenced by glycosylation [46-49].

\section{Viral Glycosylation}

Upon taking over the cellular machinery upon infection, virally produced proteins are also known to be glycosylated. In the case of HCV, a number of envelope proteins are glycosylated $[17,50]$. Further, as mentioned previously, these viral glycans assist in immune evasion, with the loss of the glycans related with increased susceptibility to antibody neutralization [17, 51, 52]. Influenza virus also present glycosylated glycans [40]. Influenza hemagglutinin (HA) initiates the infection process by binding to cellular receptors and is known to possess 
multiple glycosylation sites which has been linked to virulence in mice[53]. The Severe Acute Respiratory System Coronavirus(SARS-CoV) possesses a number of glycosylation sites, particularly in the S glycoprotein necessary for cellular binding[54, 55]. Ebola virus possesses both $\mathrm{N}$ - and O-linked glycans[56, 57].

\section{HIV-1 - A virus possessing Oligomannose N-linked glycans}

HIV-1, a lentivirus of the retrovirus family, continues to spread worldwide [58]. HIV's genome consists of nine genes[59]. Six genes in the HIV genome: tat, rev, nef, vif, vpr and vpu code for viron processing [59] Whereas, Gag, Pol, and Env encode structural and functional proteins [59]. Many of the proteins produced are precursors that require additional modification. Gag encodes protein which is cleaved into matrix (p17), capsid (p24), nucleocapsid (p7), and p6 [59]. Similarly replication proteins associated with cDNA synthesis and cDNA integration are generated as precursors cleaved to produce viral protease, reverse transcriptase and integrase [59].

Virion surface glycoproteins gp120 and gp41 are encoded on the Env gene. These glycoproteins are initially synthesized as a $160 \mathrm{kDa}$ glycoprotein precursor protein, requiring cleavage before assembly into a spike [59]. Following glycosylation in the endomembrane system, gp160 is enzymatically cleaved into gp120 and gp41 [43]. Trimerization of three molecules of gp41 with gp120 molecules spikes on the viral envelope for interacting with CD4 receptor molecules [59].

GP120 structure and glycosylation 
Gp120 protein of HIV is highly glycosylated, with an average of 24 glycosylation sites occupied [55]. Further, those glycans are almost entirely of the high-mannose variety $[13,60]$. These glycans can greatly impact viral infectivity and fitness. For example, an increase in potential $\mathrm{N}$-linked glycosylation sites can result in increased viral fitness by obscuring binding sites recognized by antibodies [56]. Additionally, viruses isolated from early time points in infection tend to be less heavily glycosylated than viruses isolated from later time points $[57,58]$.

\section{HIV Infection}

Cellular binding is the first step in the process of HIV infection. Viral particles with reduced numbers of $\mathrm{N}$-linked glycans infect activated $\mathrm{T}$-cells and dendritic cells in the genital mucosa [61-64]. CD4+ T cell infection results in migration of the virus to gut-associate lymphoid tissues $[65,66]$. There acute infection takes hold as gut-associated T-cells possessing the $\alpha 4 \beta 7$ gut homing integrin are infected and depleted [64].

The routes of sexual exposure and transmission may vary. Vaginal transmission is the most common route of transmission worldwide, though in the United States the majority of new infections occur via rectal exposure[67]. In vaginal transmission, the cervix is a major site for HIV-1 transmission as rich in HIV-1 target cells [68-70]. However, receptive anal intercourse provides the greatest probability of transmission [71, 72]. Fragile rectal tissue which can be damaged by intercourse, and a large population of $T$ cells and dendritic cells 
resident in the rectal mucosa contribute to high probability of productive infection $[71,73-77]$.

The biochemical steps of cellular infection begin with gp120 present on the envelope of HIV binding with the CD4 receptor molecule expressed on the surface of mature CD4+ T cells, macrophages and dendritic cells [78-80]. Upon binding, the CD4 receptor molecule undergoes conformational changes with allow for interaction with either of two 7-transmembrane spanning chemokine receptors, CCR5 or CXCR4 [81-83]. Co-receptor engagement and binding leads to a second conformational change in the viral envelope spike, altering the state of gp41 $[84,85]$. This alteration of gp41 leads to insertion of the N-terminal domain of gp41 into the cellular membrane. This insertion triggers formation of a six-helix bundle that provides the energy for cellular fusion. [84-86]. Upon fusion, the viral capsid releases two copies of RNA, reverse transcriptase, integrase, and viral protease. Reverse transcriptase acts to convert the RNA into DNA while Integrase cleaves host DNA and integrates the new viral DNA into the host cell genome. Later, viral protease acts to cleave precursor polyproteins into functional proteins for new virion packing and assembly.

Within weeks of infection, some persons display Acute Retroviral Syndrome associated with primary HIV infection. During that period, a large burst of viral replication occurs as the immune system reacts to the infection, attempting to eliminate the virus and infected cells. During this period, nonspecific symptoms such as fever, rash, body aches occur as a consequence of immune system activation. After a number of days to weeks, symptoms 
resolve, however the immune system is remains in a persistent inflammatory state. Despite a lack of outward symptoms, a slow but steady decrease in CD4+ cells occurs over a number of years. During this time, a gradual increase in viral load accompanies the decrease in CD4+ $\mathrm{t}$ cells.

\section{Prevention Methods and Antiviral treatments}

Pharmacological strategies are currently in use for the treatment of many of enveloped viruses, with small molecule pharmaceuticals designed to block specific events in the viral life cycle. These strategies include preventing/reducing viral replication, preventing virion processing and maturation, as well as preventing viral entry into cells.

HIV-1 currently has many treatment options available for infected individuals with the goal of suppressing viral replication below the limits of detection. However, maintenance of an undetectable viral load may be difficult to maintain due to drug side effects, drug adherence, and emergence of drug resistant virions. As such, a long acting adjuvant treatment acting upon a novel drug target may be useful for those with adherence issues, or those with few treatment options left. As such, anti-viral lectins targeting N-linked oligomannose glycans present on HIV-1 gp120 spike proteins may be a new treatment option for maintaining an undetectable viral load, and thus maintaining the health benefits an undetectable viral load brings.

Drugs to suppress HIV infection currently include non-nucleoside reverse transcriptase inhibitors, nucleoside reverse transcriptase inhibitors, protease 
inhibitors, integrase inhibitors, and fusion/entry inhibitors. Collectively known as Highly Active Anti-Retroviral Therapy (HAART), these medications, when given in combination therapy, offer the potential for infected individuals to live a normal lifespan [87]. The goal of HAART is to as suppress viral load to undetectable levels. In many cases, this leads to preservation of immune function [88]. Further, due to continued thymic output, durable viral suppression is often accompanied by a restoration of CD4+ cell populations [89, 90]. However, even in those who are virally suppressed, as significant subset, known as immunological non-responders, fail to see full immune system recovery [88, 91].

Prior to the initiation of HAART, genetic testing is conducted to determine HIV-1 drug resistances. With this knowledge in hand, the typical regimen consisting of 3 or more drugs of at least two classes is chosen [92, 93]. After initiation of HAART, the majority of patients see a very rapid decline in viral load levels as well as an increase in CD4+ T cells [94, 95]. Many individuals can achieve undetectable viral loads with vigilant adherence to therapy [94]. Further, increases in CD4 cells counts can continue for years after durable viral suppression is achieved [88, 95-97]. Nonetheless, lifetime adherence to therapy is currently required.

One complicating factor in disease management is that once HIV-1 is systemically established, viral reservoirs appear to make systemic eradication of the virus impossible [98-101]. Viral reservoirs are believed to be the latent infection of long lived memory cells. However, it is also possible that a low level viremia intermittently continues or a combination of both of those factors [98- 
102]. The practical effect of this latent infection is seen through the fact that shortly after discontinuing HAART, viral load levels rebound in the majority of patients.

In addition to viral rebound after discontinuation of therapy, intervening periods of low level viral replication can occur. Despite durable suppression of viral replication, T-cell activation during times of illness or infection can cause bursts of HIV-1 replication activity in infected cells[94]. The clinical significance of these "blips" are currently not thought to be relevant[103]. However, it is possible that detectable viremia may be indicative of the acquisition of viral resistance[103].

Nonetheless, many studies now support the notion that undetectable viral loads are the primary goal of treatment. Studies have indicated that undetectable viral load levels could be even more important than CD4+ counts [104]. Thus a patient who is consistently undetectable in their viral load testing may need less periodic testing for CD4 counts [104]. As such, anti-viral agents, such as antiviral lectins, that could further prevent cell-to-cell transmission of HIV during periods intermittent or transient viremia may aid in maintaining undetectable viral loads.

Pre-Exposure Prophylaxis (PrEP)

Recently, a new prevention method was approved for usage within the United States which validates the concept of patient pre-treatment with anti-viral agents to prevent HIV-1 infection. Pre-exposure Prophylaxis (PrEP) is a 
chemotherapeutic intervention that offers the promise of preventing HIV-1 infection if used as directed $[105,106]$. PrEP currently consists of administration of antiviral drugs to HIV negative persons at risk for infection [107]. Presently, Truvada (a combination of emtricitabine and tenofovir) is the only drug combination provided to high-risk, HIV-1 negative, individuals [96]. If taken daily as directed, PrEP has been observed to substantially reduce chances of HIV-1 infection after exposure $[105,108]$. Nonetheless, PrEP is not devoid of risks. Rarely, kidney damage and malfunction can occur which would necessitate cessation of $\operatorname{PrEP}$ [109-112]. As such, frequent kidney function testing is recommended [96]. As such, this emerging field prevention method is likely to increase as additional antiviral drug interventions are validated.

\section{Treatment as Prevention (TasP)}

A second concept which underscores the need to maintain undetectable viral loads is summarized as Treatment as Prevention (TasP) [113]. Preliminary results from the PARTNER study have shown no transmissions between individuals when the positive partner has an undetectable viral load [114]. While the final results of the study will not be available for a number of years, it appears that an undetectable viral load in a positive individual strongly correlates with infectiousness $[115,116]$. As such, attaining and maintaining an undetectable viral load may prove crucial in curbing new infections. 
Post Exposure Prophylaxis (PEP)

Post Exposure Prophylaxis (PEP) has also been used successfully to prevent viral infection [117-120]. PEP consists of a month long course of antiretroviral medication after both occupational and nonoccupational exposures. With needlesticks being a common occupational exposure, PEP has been observed to be effective in preventing infection [120]. Further, in a nonoccupational exposure setting, such as condom breaks during intercourse, PEP has shown great efficacy [119]. However, it typically must be initiated within 48 hours after exposure in order to reduce the odds of transmission.

\section{Entry Inhibitors}

Entry inhibitors that prevent viral fusion with its target cell at various phases of the viral entry process are currently being used in treatment and research. These agents target proteins on either the HIV-1 virus or the cell. Targets include: gp120 of HIV-1, gp41 of HIV-1, and T-cell receptors and coreceptors. Entry inhibitors mechanistically present a barrier to infection through binding activity to viral or cellular receptors necessary for infection [33, 121].

Entry inhibitors that neutralize the virus, such as isolated broadly neutralizing monoclonal antibodies, are potential chemoprophylactic and therapeutics for HIV-1. These broadly neutralizing antibodies recognize epitopes (such as gp41, V1V2-glycans, and the CD4 binding site) on the HIV-1 envelope and can neutralize multiple strains. For instance, antibody b12 recognized the 
CD4 binding site of HIV-1 [122], whereas 2G12 recognizes the outer domain glycan [123]. Other monoclonal antibodies, such as 3BNC117 and 10-1074, which target the CD4 binding site and $\mathrm{V} 3$ region respectively, have been examined both as monotherapy and together in a Macaque SHIV models [124]. When used individually, emergence of neutralization-resistant variants often limits their long term utility [124]. However, broadly neutralizing may hold promise when used in cocktails to treat chronic HIV infection [124, 125].

While many entry inhibitors directly act on the virus, an alternative approach may include the competitive blockage of receptor or co-receptor molecules on HIV target cells. For example, Ibalizumab is a nonimmunosuppressive CD4-targeting IgG molecule [126-128]. Ibalizumab binds the CD4 molecule present on T-cells receptors in the D2 region $[129,130]$. While blocking portions of the CD4 receptor, it does not interfere with binding of MHC II molecules or the gp120-- thus it does not interfere with normal immune function [129-131]

Enfuvirtide, is a 36 amino acid peptide mimic of the HR2 fragment of gp41. Functionally it binds to the HR1 region of gp41 [132, 133]. Enfuvirtide binding blocks formation of the six helix bundle required for the cellular fusion process [85]. While it is an injectible peptide fusion inhibitor [134], it was approved for treatment of HIV infection in 2003 [135].

Maraviroc is CCR5 co-receptor antagonist [136-138]. Maraviroc binds to the transmembrane cavity of the CCR5 co-receptor, halting the co-receptor interactions necessary for viral fusion [139]. 


\section{Entry Inhibitors - Antiviral Lectins}

Lectins are also being investigated as potential HIV-1 entry inhibitors [22]. Lectins are highly specific carbohydrate binding proteins. In animals, lectins can function in the immune system recognition of carbohydrates exclusively presented by pathogens. For example, mannose binding lectin is protein of the innate system that activates complement or triggers pathogen opsonization [83].

Antiviral lectins consist of a subset of lectins which bind to viral envelope glycans [22]. Among these are Griffithsin (GRFT), Scytovirin (SVN), Bananna lectin (BanLec), Concanavalin A (ConA), Cyanovirin-N (CVN), and Actinohivin [27, 140-142]. While different lectins have different carbohydrate targets, such as high-mannose glycans or complex glycans, lectins specific for HIV glycans are thought to bind high-mannose glycans of gp120, preventing cell fusion [21, 22, 143].

Despite their promise, lectin therapy may be challenging. First, exogenous lectins, like Phytohemagluttinin (PHA), are well known to agglutinate red blood cells [144] and often induce mitogenic and cytotoxic effects [145]. Second, lectins are often considered anti-nutrients given their sugar binding activities [146]. For example, large consumption of lectins can cause side effects such as diarrhea, nausea, and vomiting [147]. Finally, lectins may interact with leptin receptors, leading to leptin resistance [148].

In vitro studies with antiviral lectins have also identified a number of potential issues. For instance, studies into CVN, which binds to alpha $(1,2)$ mannose termini, have identified cell morphology changes in PBMC's treated 
with CVN as well as increased expression of cellular activation markers [149]. These changes rendered the PBMCs more susceptible to HIV-1 infection by R5 trophic variants. Further, CVN was found to increase production in a number of cytokines. BanLec, a potent anti-HIV-1 lectin with specificity for oligomannose glycans higher than (Man)6GlcNAc, has been found to be an agglutinating agent in rabbit erythrocytes as well as a powerful murine T-cell mitogen [150, 151].

In vivo studies with antiviral lectins has also identified challenges. ConA, which has an anti-HIV-1 $50 \%$ effective concentration $\left(\mathrm{EC}_{50}\right)$ of approximately 98nM has significant in vivo toxicity [25]. ConA binds high-mannose type, hybrid type and biantennary complex type $\mathrm{N}$-glycans [152] and has been observed to strongly agglutinate many cell types, as well as has been identified as having fetal development toxicity in rats [153]. Further, investigation into ConA's toxicity identified ConA as inducing focal hepatic necrosis, lymphoid atropy, and vascular endothelial damage at a $200-800 \mu \mathrm{g}$ dosage in mice $[154,155]$.

In addition to potential toxicities associated with carbohydrate binding activity, lectins may induce allergic reactions and anaphylaxis. Immune reaction to exogenous proteins are not uncommon [156]. The largest potential threat is anaphylaxis [82]. However, other, less life threatening immune reactions may also occur, such as the generation of neutralizing antibodies [82].

Protein therapy-induced anaphylaxis has been observed with approved therapeutics Rituximab, Omalizumab, and alpha galactosidase A [157-160]. These therapeutics are testament to the idea that despite the risks, the benefits of protein based therapy can weigh in favor of approval. Nonetheless, efforts to 
reduce the chance of lethal reactions continue. For example, efforts into structure guided protein modifications to reduce immunogenicity have been theorized [161, 162]. These modifications would lessen the potential for immune system recognition, thus reducing the possibility of an immune response $[161,162]$.

\section{Griffithsin Structure and Mechanism of action}

GRFT is an antiviral lectin derived from the red algae Griffithsia $s p$, possessing a strong affinity for $\mathrm{N}$-linked high mannose glycans [32, 33]. GRFT forms $\sim 25 \mathrm{kDa}$ domain swapped homodimers in solution, with the first 16 amino acids of each monomer completing the B-prism fold of the other monomer [163]. These jacalin-like binding pockets are formed by 3 , four stranded beta sheets, with an internal symmetry. A total of 6 mannose binding sites have been identified by X-ray crystallography of dimeric GRFT at $1.3 \mathrm{~A}$ resolution $[163,164]$. The six carbohydrate binding pockets are equally split, with three carbohydrate binding pockets being located on opposite ends of the double-prism structure [163]. Atomic resolution crystal structures of an engineered monomeric GRFT have revealed that each monomer can bind to two different nonamannoside molecules through all three carbohydrate binding sites [163, 165]. Further, structural complexes of GRFT with saccharides have identified 3 virtually identical active sites on each monomer consisting of a GGSGG motif [166]. Upon dimeric folding, X-ray crystallography has also identified the geometries of the binding sites as being equilaterally triangular in shape [164]. 
In the case of HIV-1, GRFT's inhibitory activity is associated to its binding of the almost exclusively high-mannose glycans present on gp120 protein [13, 32]. Supporting this theory is the fact that lectin-resistant isolates of HIV have reduced numbers of glycosylation sites [16]. Additionally, a loss in antiviral activity was observed with monomerization of GRFT, suggesting that GRFT's ability to cross link virions or gp120 proteins is key to its antiviral activity[167]. This crosslinking of $\mathrm{N}$-linked glycans present on the envelopes is thought to inhibit structural transitions required for viral entry. GRFT has also been observed to inhibit HIV-1 transfer from dendritic cells (DC) receptor DC-SIGN to uninfected T-cells. To date, GRFT is the most potent antiretroviral drug of any drug class for preventing transmission of cell-free and -associated HIV-1.

Prior work has suggested that GRFT can be cost effectively produced in tobacco plants $[168,169]$. Additionally, prior studies have suggested GRFT's weak immunogenicity and demonstrated low induction of inflammatory cytokines and chemokines in animal models [33, 169]. These studies have confirmed GRFT displays no human T-cell mitogenic activity, and unlike many other lectins does not induce production of pro-inflammatory cytokines in treated human peripheral blood mononuclear cells at $>10,000$ times antiviral concentrations [35, 169].

GRFT also has a notably broad spectrum of activity. Antiviral activity against HCV [30, 170], HIV-1 [32, 169, 171], HIV-2 [171], and SARS-CoV [172] has been documented. Unpublished studies have also demonstrated activity versus influenza A virus, and several other enveloped viral pathogens (O'Keefe 
$\mathrm{BR}$, unpublished). For many of these diseases, GRFT has a markedly low in vitro inhibitory activity. Against HCV, GRFT's $\mathrm{EC}_{50}$ has been found to be approximately $13.9 \mathrm{nM}$ [170]. Versus SARS-CoV, it has an $\mathrm{EC}_{50}$ of $48 \mathrm{nM}$. [163, 172]. However, in the case of HIV-1, Griffithsin has an in vitro $\mathrm{EC}_{50}$ in the mid picomolar to low nanomolar range [16, 32, 169].

General studies into GRFT's systemic tolerance have also been encouraging. We observed GRFT to be tolerable during a 10 day chronic dosing regimen [34]. This tolerability, seen in both mice and guinea pigs subcutaneously dosed with GRFT raises the potential for short term usage of GRFT as a prophylactic treatment. Further, continued antiviral activity against HIV-env pseudoviruses and a long serum persistence of GRFT were observed [34]. These factors would support in vivo administration of GRFT in an exposure setting wherein amelioration or prevention of establishment of infection were the goal of therapy.

GRFT's antiviral activity has also been observed both ex vivo and in vivo. HIV-1 infection of human cervical explants has been prevented by topical application [169]. In vivo challenges in mice have also been positive, with activity versus: SARS-CoV (intranasal treatments) [172]; Japanese encephalitis virus (JEV) (intraperitoneal treatment) [173]; and HCV (subcutaneous treatment mouse-human chimeric liver models) [30, 170].

With the short term tolerability of GRFT administration established, as well as the potential for viral inhibition in vivo, it is possible that GRFT may be useful in established infections. Despite the advent of modern HAART, which can 
suppress viral replication below currently detectible limits, new therapies are constantly needed. HIV's propensity for mutation under drug pressure translates into an eventual viral breakthrough. Further, as mentioned previously, during times of immune activation-such as a cold or infection, viral "blips" may occur in which a person with a previously undetectable viral load displays a detectable viremia[94, 103]. Accordingly, agents which could further reduce the chance of drug resistant isolate proliferating may assist in maintaining viral undetectable viral loads - a key factor in infectiousness. Further, given the current window of opportunity for preventing establishment of a productive infection, additional drugs that could be used for PrEP or PEP may have utility in both occupational and nonoccupational settings. However, for GRFT's usage in established infections, long term systemic impacts, including potential immune activation which may trigger low level viremia, a neutralizing antibody response, or systemic toxicity, need to be assessed. 


\section{OVERARCHING HYPOTHESIS}

This work presents a compilation of work investigating the overarching hypothesis that GRFT is a candidate for systemic antiviral therapy. However, a number of unknowns currently exist. In Chapter 2, we review the animal studies which laid the foundation for the present evaluation of GRFT's systemic tolerance. In Chapter 3, we examine the practical pharmacokinetics of GRFT in Sprague Dawley rats and its potential impact upon method of administration, timing of dosage, and method of excretion. In Chapter 4, we examine the tolerability of sub-chronic dosing of GRFT in Sprague Dawley rats, with an immune response primed by pre-immunization with GRFT as well as passive immunization with anti-GRFT IgG. Finally in Chapter 5, we examine GRFT's biochemical interactions through Differential Scanning Fluorometry and Affinity Purification, as well as examination of the effects on endogenous proteome interactions through Differential Scanning Calorimetry. The findings in this Dissertation lay a foundation for further study to validate GRFT's usage as a systemic antiviral therapeutic or prophylactic. 


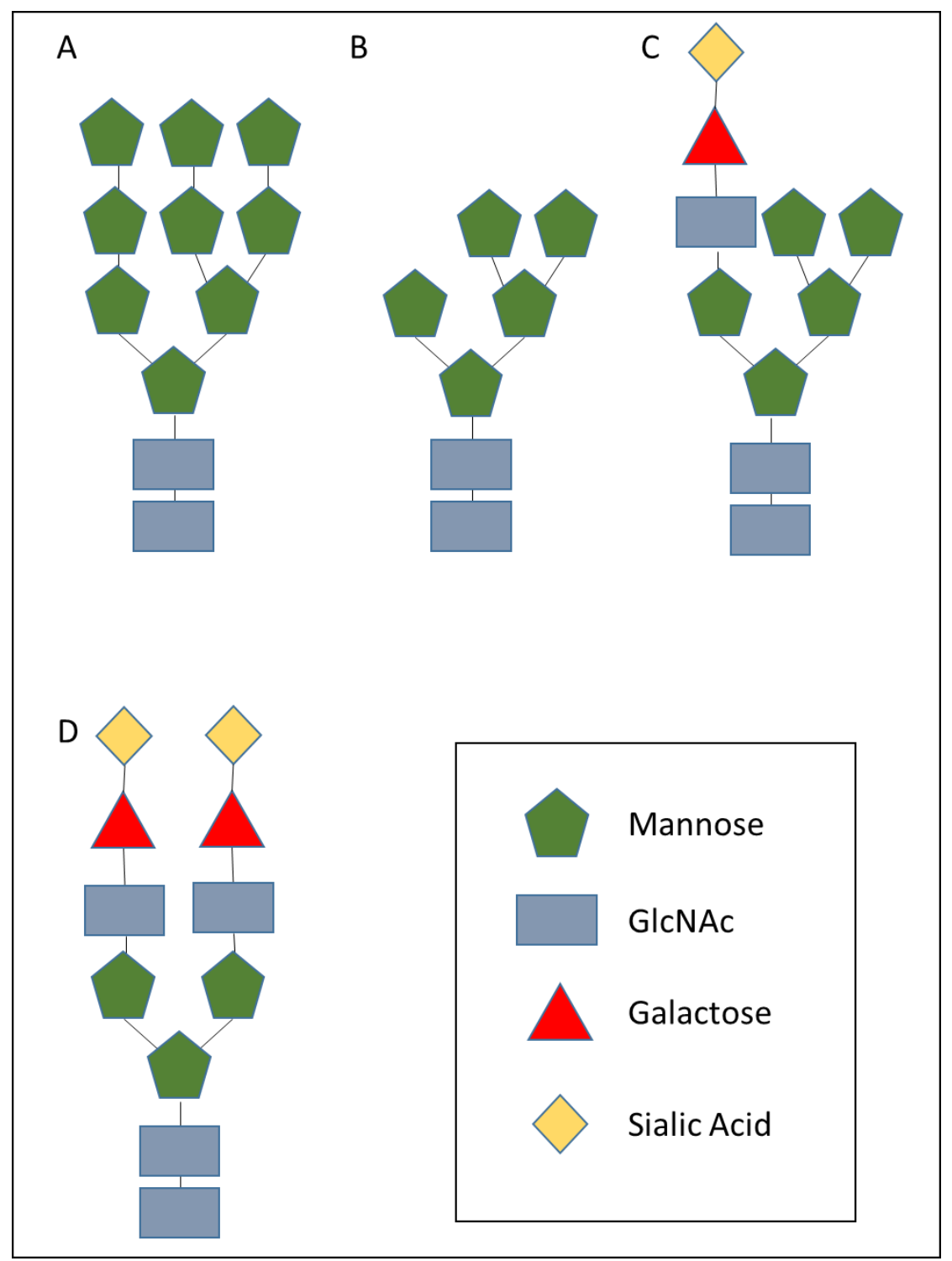

Figure 1.1 N-linked Glycans - structural arrangement of subtypes. Schematics illustrating examples of different N-linked glycans. Oligommanose Glycans Man9 (A) and Man5 (B) present exclusively mannose termini. Hybrid Glycans (C) present both mannose termini and GlcNAc termini. Complex glycans (D) have no mannose termini. 


\section{CHAPTER 2}

\section{ACTIVITY AND EFFECT OF SUBCUTANEOUS TREATMENT WITH THE BROAD SPECTRUM ANTIVIRAL LECTIN GRIFFITHSIN IN TWO LABORATORY RODENT MODELS ${ }^{1}$}

\section{INTRODUCTION}

The glycan structures displayed on envelope glycoproteins frequently play important roles in virus transmission and entry into target cells [40]. Viruses that establish chronic infections, such as HIV and HCV, display a dense shield of oligomannose glycans that also assist the pathogen in immune evasion, both through the display of "self"-like epitopes as well as by induction of immunosuppressive innate immune responses $[13,17,19,51,60]$. Antiviral compounds that target envelope glycoproteins are classified as "carbohydrate binding agents" (CBA), and generally encompass lectins and non-peptidic antibiotics such as pradimicin $\mathrm{A}$ and $-\mathrm{S}$ and benanomicin $\mathrm{A}$ (reviewed by Balzarini [14]). Several different lectins from natural sources show significant antiviral activity in vitro and have been proposed as antiviral prophylactic and

\footnotetext{
${ }^{1}$ Antimicrob Agents Chemother. 2014 Jan;58(1):120-7.
} 
therapeutic compounds. While there is a plethora of publications in the scientific literature showing in vitro antiviral activity of CBA against a broad array of enveloped viruses, most in vivo studies on safety and efficacy of this class of compounds are in pre-exposure prophylaxis models. However, two important studies demonstrate the potential of antiviral therapy with lectins. Smee et al. [28] demonstrated that post-exposure treatment with the antiviral lectin cyanovirin- $\mathrm{N}$, which targets an $\alpha-(1-2)$-linked mannobiose substructure on oligomannose glycans, showed significant survival benefit 6 hours after infection in a murine influenza model. A recent study by Michelow and colleagues [29] demonstrated that high dose therapy with human mannose binding lectin (MBL), an endogenous C-type lectin that recognizes glycan structures including mannose, glucose and fucose on the surface of pathogens, could ameliorate Ebola virus infection in a murine model. Despite their demonstrated antiviral activities, the fact that many natural product lectins have significant in vitro and in vivo toxicity, acting as non-specific T-cell stimulants and red blood cell agglutinating agents, has limited their development as antiviral therapeutics. However, not all antiviral lectins are toxins and not all antiviral lectins have cell agglutinating activity.

Griffithsin is a $12.77 \mathrm{kDa}$ red algae derived lectin, that binds the terminal mannose residues on the asparagine $(\mathrm{N})$-linked Man5-9GIcNAc2 structures that comprise the vast majority of $\mathrm{N}$-linked glycans in the HIV-1 glycan shield [32, 163-165]. GRFT displays no human T-cell mitogenic activity, and unlike many other lectins does not induce production of pro-inflammatory cytokines in treated 
human peripheral blood mononuclear cells $[35,169]$. In collaborative studies, we have shown that GRFT has broad spectrum antiviral activity against HIV-1 [32, 169, 171], HIV-2 [171], HCV [30, 170], an array of pathogenic coronaviruses, including SARS-CoV [172], in addition to influenza A virus, and several other enveloped viral pathogens (O'Keefe BR, unpublished). The in vitro inhibitory activity $\left(\mathrm{EC}_{50}\right)$ of GRFT against HIV-1 is in the mid picomolar to low nanomolar range, for most isolates $[16,32,169]$. GRFT has $\mathrm{EC}_{50}$ against $\mathrm{HCV}(13.9 \mathrm{nM})$ [170] and SARS-CoV (48 nM) [163, 172]. There is a growing body of published evidence that GRFT also has antiviral activity ex vivo as well as in vivo: topical application of GRFT prevents HIV-1 infection of human cervical explants [169]; intranasal treatments with GRFT prevents disease in mice challenged with SARS-CoV [172]; intraperitoneal (IP) treatment with GRFT prevents Japanese encephalitis virus infection in mice [173]; and subcutaneous treatment with GRFT shows some efficacy against HCV challenge in a mouse-human chimeric liver models $[30,170]$. The JEV and HCV studies demonstrated that GRFT is relatively well tolerated by mice exposed to the drug systemically at $5 \mathrm{mg} / \mathrm{kg}$ doses, but did not report a comprehensive assessment of GRFT safety as a potential systemic antiviral treatment. Here, we report that minimal toxicity is induced by a range of subcutaneous doses of GRFT in two rodent species. The drug was systemically distributed, and accumulates to high levels in the serum and plasma after subcutaneous delivery. Furthermore, we demonstrated that serum from GRFT treated animals retained anti-viral activity against HIV-1 enveloped pseudoviruses in a cell-based neutralization assay. Overall, these 
findings support further investigation into GRFT's potential as a systemic antiviral therapeutic agent against enveloped viruses including HIV-1. 


\section{MATERIALS AND METHODS}

\section{Lectin reagents}

Recombinant GRFT was produced in Nicotiana benthamiana plants as described previously, purified to $>99 \%$ purity, and formulated in phosphate buffered saline (PBS), pH 7.4 [169]. Phytohemagglutinin A (PHA) was purchased from Sigma.

\section{Animal housing and care}

6-8 week-old female BALB/c mice (Jackson Laboratory) and Hartley guinea pigs (Cavia porcellus, Charles River Laboratories) were housed in a temperature- and humidity-controlled room with an alternating light/dark cycle of $12 \mathrm{~h}$, with standard diet and water ad libitum. All experimental procedures were approved by the University of Louisville's Institutional Animal Care and Use Committee.

\section{Mouse treatments and sample collection}

To evaluate effects of a single high dose of GRFT, mice were injected subcutaneously with $50 \mathrm{mg} / \mathrm{kg}$ GRFT $(n=30)$ or PBS $(n=15)$. At 1,7 , and 14 days post-treatment 10 mice treated with GRFT and 5 control animals were sacrificed and blood was collected by cardiac puncture. Kidneys, livers, and spleens were excised. For chronic administration, mice were treated with 10 $\mathrm{mg} / \mathrm{kg}$ GRFT $(n=15)$ or PBS $(n=15)$ daily for 14 days. Each group was further subdivided into 3 groups of 5 mice each. Blood was collected from the submandibular vein every other day, alternating between subgroups. Animals were sacrificed on day 14 (9 mice per treatment group), day 16 and day 21 (3 
mice per treatment group at each time point), and the samples collected as outlined above.

\section{Guinea pig treatment and sample collection}

For chronic administration in guinea pigs, two studies were conducted at different times. In the first experiment, $10 \mathrm{mg} / \mathrm{kg}$ GRFT ( $\mathrm{n}=12$ ) or $1 \mathrm{ml} / \mathrm{kg}$ PBS ( $\mathrm{n}=6)$ was subcutaneously administered daily for 10 days. The second experiment was similar to the first, except for the number of animals (GRFT, $n=10$; PBS, $n=6$ ). Half of the animals in each group were sacrificed on day 11 after cardiac puncture exsanguination under isoflurane anesthesia, and the remaining animals were euthanized on day 15 . Blood and organs were collected at sacrifice.

\section{Extraction of GRFT from mouse organs}

100-500 mg pooled organ tissues were homogenized in $1 \mathrm{ml}$ PBS supplemented with complete, EDTA-free Protease Inhibitor Cocktail (Roche) and the samples cleared by a series of two centrifugations steps at respectively 10,000 and $15,000 \times \mathrm{g}$ for $10 \mathrm{~min}$ each. The supernatants were stored at $-20^{\circ} \mathrm{C}$ until use. GRFT capture immunoassay using the HIV-1 envelope glycoprotein gp120

To detect trace amounts of GRFT present in serum and plasma, and in homogenized organ tissues, we used a HIV-1 gp120 binding ELISA as previously described [35] with a few modifications. Briefly, Maxisorp plates (Nunc) were coated with $25 \mathrm{ng}$ purified gp120 (Protein Sciences) and incubated overnight at $4^{\circ} \mathrm{C}$. Plates were blocked with $3 \%(\mathrm{w} / \mathrm{v})$ Bovine Serum Albumin (BSA) in PBS 
containing $0.05 \%$ Tween 20 (PBS-T). Samples were diluted 1:10 in blocking buffer and were incubated at RT for 1 hour. Serial dilutions of purified GRFT were run in parallel to generate a standard curve. The gp120-bound GRFT was detected by rabbit anti-GRFT antiserum $(1: 25,000)$ followed by HRP-conjugated goat anti-rabbit $\lg G(1: 10,000)$. Plates were developed with SureBlue TMB Microwell Peroxidase Substrate, and reactions were stopped with $1 \mathrm{~N} \mathrm{H}_{2} \mathrm{SO}_{4}$. Absorbance readings at $450 \mathrm{~nm}$ and $570 \mathrm{~nm}$ were measured using a BioTek Synergy HT plate reader.

\section{Evaluation of Anti-HIV Activity.}

HIV-1 neutralization activity of heat-inactivated serum or plasma was measured using pseudovirus neutralization assays as previously described [174]. Briefly, molecularly cloned DU156 env-pseudotyped virus particles were generated by transfection of 293T cells and titrated in TZM-bl cells. Antiviral activity was measured as a function of luciferase reporter gene activity. $I D_{50}$ values were defined as the sample dilution required to reduce luminescence by $50 \%$ in comparison to wells with no sample added (within the linear range of the assay).

Hematology parameters and Serum Chemistry.

A complete blood count $(\mathrm{CBC})$ was run for guinea pig samples using a Hemavet $\AA 950$ (Drew Scientific) standardized for guinea pig blood. The following parameters were quantified in potassium-EDTA anticoagulated whole blood: red blood cells (RBC; $\left.10^{4} / \mu \mathrm{l}\right)$, total and differential leukocyte count (neutrophils, lymphocytes, monocytes, eosinophils, and basophils as $10^{3} / \mu$ or $\%$ ), hemoglobin 
concentration (HGB; g/dl), hematocrit (HCT; \%), mean corpuscular volume $(\mathrm{MCV} ; \mathrm{fl})$, mean cell hemoglobin $(\mathrm{MCH} ; \mathrm{pg})$, mean cell hemoglobin concentration (MCHC; g/dl), red cell distribution width (RDW; \%), platelets (PLT; $\left.10^{4} / \mu \mathrm{l}\right)$, and mean platelet volume (MPV; fl).

In the first guinea pig experiment levels of the following serum chemistries were assessed and the differences analyzed by two-way ANOVA: serum albumin (Alb), alkaline phosphatase (ALKP), amylase (Amy), alanine aminotransferase (ALT), blood urea nitrogen (BUN), calcium (Ca), cholesterol (Chol), creatinine (Creat), globulin (Glob), glucose (Glu), phosphorus (Phos), total bilirubin (TBil), and total protein (TP). Based on the results obtained, we decided to further measure the effect of GRFT on selected markers including serum albumin, alkaline phosphatase, and amylase in the second experiment using a VetTest $\circledast$ Chemistry Analyzer (IDEXX Laboratories)

\section{Hemagluttination assays.}

Guinea pig, sheep, and human blood (Innovative Research), and blood collected from untreated mice was washed and resuspended at a final concentration of 1 $2 \%(\mathrm{v} / \mathrm{v})$ in $1 \mathrm{X}$ PBS containing $3 \mathrm{~g} / \mathrm{L}$ BSA and $1 \mathrm{~g} / \mathrm{L}$ sodium azide. PBS, PHA, or GRFT were mixed with an equal volume of erythrocytes in a 96-well round bottom plate. The plate was incubated for $1 \mathrm{hr}$ at $\mathrm{RT}$, followed by overnight incubation at $4^{\circ} \mathrm{C}$. Finally, wells were dried and hemagglutination activity was determined by visual examination. 


\section{Statistical Analysis.}

Statistical analysis was conducted using Graph Pad Prism 5 and SAS software version 9.3. Because of the stratification by guinea pig study, day of sacrifice and treatment group, it was decided to increase statistical power by using all the data from both studies. Three-way ANOVA [175] analysis was utilized, which allowed testing for treatment effect while at the same time adjusting for differences between the two studies and the days of sacrifice. For initial univariable analysis, the two-sample t-test [176] or Wilcoxon rank sum test [177] was used to test for differences between study, day of sacrifice, and treatment. For data sets collected in only one guinea pig study - such as weight change and non-selected serum chemistries, a two-way ANOVA was utilized. A p-value < 0.05 was deemed significant. 


\section{RESULTS}

\section{Griffithsin serum concentrations.}

Plasma samples collected from mice injected with a single high dose of $50 \mathrm{mg} / \mathrm{kg}$ GRFT showed up to $4 \mathrm{nM}$ GRFT ( $4 \mathrm{nM}$ equals $51.08 \mathrm{ng} / \mathrm{ml}$ ) in animals sacrificed one day post treatment (Figure 2.1A). These levels decreased considerably to less than $0.5 \mathrm{nM}$ by day 7 , and persisted through day 14 .

In chronically dosed mice, plasma concentrations of GRFT peaked at $25 \mathrm{nM}$ (Figure 2.1B) by day 11, followed by a gradual decrease in detectable GRFT even with subsequent treatments. This trend continued throughout the recovery time. Notably, concentrations of GRFT persisted in the plasma at levels of approximately $4 \mathrm{nM}$ after a week of recovery (Figure 2.1B). GRFT concentrations in sera from chronically treated guinea pigs were similar to that of chronically treated mice, with mean concentrations of $36 \mathrm{nM}$ at day 11 and 11.36 nM at day 15 (Figure 2.1C).

Anti-HIV Activity of plasma and serum.

Plasma samples collected from mice treated with a single dose of $50 \mathrm{mg} / \mathrm{kg}$ GRFT neutralized HIV-1 pseudoviruses (Clade C primary sexually transmitted isolate Du156) with a mean $\mathrm{ID}_{50}$ (dilution required for a $50 \%$ reduction in luminescence versus controls) of 1500 on experimental day 2 (Figure 2.2A).

This neutralization activity decreased in samples obtained at days $8\left(\mathrm{ID}_{50}\right.$ of 300$)$ and $15\left(\mathrm{ID}_{50}\right.$ of 200$)$. Plasma from chronically treated mice neutralized the HIV-1 Du156 pseudovirus with an $I_{50}$ value of approximately 800 (Figure $2.2 B$ ) at day 
14. After the 7 day recovery period, the $I D_{50}$ values decreased to approximately 200. Serum samples collected from guinea pigs after chronic treatment with 10 $\mathrm{mg} / \mathrm{kg}$ GRFT displayed a mean ID 50 of approximately 3277 at day 11 and 576 at day 15 , respectively. The HIV-1 Du156 neutralization activity of guinea pig sera correlated well with the concentration of GRFT detected in the serum; however, the GRFT serum concentrations detected in mouse samples predicted only about $14-25 \%$ of the actual serum HIV-1 neutralization activity we observed, confirming that additional non-specific HIV-1 neutralizing activities in mouse serum contribute to the higher-than expected overall HIV-1 inhibitory activity in the mouse sera.

GRFT distribution into tissues.

Organs from mice chronically treated with GRFT were harvested, and total protein extracted. We measured the total amounts of GRFT that accumulated in these tissues by gp120 binding ELISA. Figure 2.3 shows that GRFT accumulated in all three organs assayed, with most GRFT accumulating in the spleen. Non quantitative immunofluorescence studies detected GRFT in the same organs (liver, kidney and spleen) harvested from treated guinea pigs (data not shown).

GRFT is tolerated after subcutaneous administration

We studied the toxicity of GRFT in guinea pigs using several parameters including mortality, behavior, animal body and organ weight changes, tissue pathology, and changes in blood properties. 
All animals survived and no change in behavior was observed. Since the guinea pigs used in this work were juvenile, we evaluated their overall fitness after GRFT treatment using body weight as a surrogate marker. Animals were weighed at day 1 and at time of sacrifice. Using a 2-way ANOVA to measure the impact of treatment and time on bodyweight gains, we found that GRFT treatment resulted in significantly less bodyweight gain in comparison with PBS treated controls $(p=0.0011$, Figure 2.4)

Liver, kidney, and spleen weights measured at termination were normalized to total body weights and compared to time matched controls. A statistically significant increase of the normalized weights of guinea pig livers and spleens was observed for GRFT treated animals $(p=0.008$ and 0.005 , respectively, Figure 2.5). Tissue sections from guinea pigs in the second experiment were stained with hematoxylin and eosin and evaluated in a blinded fashion by a veterinary pathologist (O.F.). No distinct pathologies were observed as a result of GRFT treatment. Of all CBC parameters tested, a statistically significant difference was observed only in Red Blood Cell Width (RDW) $(p=0.016$, Table 2.1). Of note the RDW values obtained for GRFT-treated animals were still within the normal physiological range described for guinea pigs (38).

In addition to CBCs, serum chemistries were examined. When data collected from both experiments were combined for analysis, a statistically significant increase was observed for Alkaline Phosphatase after GRFT treatment compared with controls ( $p=0.001$, Figure 2.6$)$. There were no statistically significant differences between the GRFT treated group and the PBS control 
group in the remaining serum chemistry when examined by 2-way ANOVA (Figure 2.6).

Since many natural product lectins cause hemagglutination, we investigated GRFT's hemagglutination activity in several species. Blood samples from guinea pig, mouse, sheep, and human were tested. Interestingly, only red blood cells from guinea pigs were affected by GRFT—at concentrations over $5 \mu \mathrm{g} / \mathrm{ml}$ (Fig. 2.7). As expected, the vehicle (PBS) did not show any hemagglutination activity on erythrocytes, and the known hemagglutinating agent PHA demonstrated activity at concentrations of $5 \mu \mathrm{g} / \mathrm{ml}$ and above for all species tested. 


\section{DISCUSSION}

In this study, we demonstrate that GRFT persists in serum and plasma of laboratory rodents at concentrations well above the $\mathrm{EC}_{50}$ described for several known enveloped viruses $[23,32,170,178]$ after subcutaneous administration. Although decreasing in concentrations after final administration, functionally active concentrations of GRFT, as determined by gp120 binding ELISA, remain in circulation for many days after treatment cessation both in single and chronic dosing regimens. These findings support further investigations of the utility of GRFT in treatment of both acute and chronic viral infections.

Every drug candidate must show a favorable safety profile to advocate for its further development. Previously, we demonstrated that GRFT was devoid of any mitogenic and cytotoxic activity, was unable to induce cell mediators of inflammation, and had only minimal off target effects on human cells [35] corroborating with other works $[32,169]$ and unlike other anti-HIV lectins including CVN and ConA $[149,179]$. In the present in vivo studies GRFT did not alter experimental animal behavior and no animal died as a result of treatment. Using juvenile animals, we did notice that guinea pigs treated with GRFT gained weight significantly slower than those injected with PBS. This is not the case when adult mice are treated s.c. with GRFT (manuscript in preparation). Organ toxicity was also assessed both by measuring weights and histopathology. While liver and spleen percentages displayed a statistically significant increase in comparison with controls, histopathological examination of the organs from GRFT treated animals did not show any pathology. 
When a complete blood count was performed and serum chemistries analyzed, we observed that most of the parameters were not significantly changed as a result of GRFT treatment. The only exception was RDW. Although significantly different from PBS controls, RDW values obtained from GRFTtreated animals remained within the normal range described for guinea pigs. In serum chemistries, alkaline phosphatase was significantly elevated in GRFT treated guinea pigs. Whether this elevation is related to the increased liver mass to body weight ratio seen with GRFT-treated animals is yet to be determined since the alkaline phosphatase isotype was not determined.

As a xenogeneic protein, a key concern is a possible immune response to GRFT which could lead to anaphylaxis [180]. Our data showing treatment associated increases in spleen and liver mass to body weight ratios is suggestive of a nascent immune response to GRFT treatment. However, we were unable to detect anti-GRFT antibodies in sera from these treated animals, probably reflective of the short duration of these studies. Although GRFT is a relatively weak immunogen, we are able to raise high titer antibodies in animals immunized with GRFT in the presence of adjuvant; these hyperimmune sera are binding, but non-neutralizing (data not shown). Immunogenicity of biologic drugs is a widely acknowledged issue, and our data suggest that future efforts to de-immunize GRFT by structure-guided elimination of T-cell epitopes [161, 162], may be necessary before the product is used for chronic treatment of viral infection in humans. Lectins are well known for their mitogenic and agglutinating properties $[149,151,179,181-183]$ which prevent their use as therapeutics. We have 
addressed the mitogenicity concern by showing previously that GRFT lacks Tcell mitogenic activity [35]. Here, we demonstrated that GRFT does not agglutinate red blood cells from several species including human, mouse and sheep. However, we observed that guinea pig erythrocytes were agglutinated by GRFT at high concentrations. The cause of this discrepancy is unclear, but may well be associated with the treatment-associated increase in red blood cell width we observed. We conclude that the preliminary toxicity profile of GRFT is acceptable and favors its further development in antiviral prophylaxis and therapy.

GRFT is currently under development as a topical microbicide as well as a broad spectrum antiviral. However GRFT is not systemically bioavailable after topical administration (our unpublished observations), so parenteral administration is probably necessary to achieve sufficient drug in systemic concentrations necessary for effective suppression of viral replication. Our data confirm that subcutaneous administration of GRFT is a viable and efficient way to get the drug into circulation to allow a sustained pharmacodynamic effect [184]. Our data support the results of published studies on GRFT prophylactic efficacy in murine models of HCV and JEV infection[30, 31, 173]. Both studies found that GRFT effectively suppressed viral replication when administered at a $5 \mathrm{mg} / \mathrm{kg}$ dose, either subcutaneously or intraperitoneally. We are currently studying whether administrations of GRFT, under the $10 \mathrm{mg} / \mathrm{kg}$ dosage, are sufficient to maintain drug concentrations at potentially therapeutic levels. There is substantial precedent for patient self-administration of drugs via the 
subcutaneous route; indeed the peptide HIV fusion inhibitor Enfurtide (T-20) is dosed in this fashion. Our data confirm that we can achieve HIV-1 Du156 50\% serum neutralization indices in excess of 500 after 14 daily doses of GRFT at 10 mg/kg (Fig. 2.2), which should be sufficient to inhibit HIV replication, and perhaps promote viral evolution towards enhanced humoral antibody suppression[14, 151, 184]. These levels were also more than sufficient to prevent JEV and HCV infection in respective mouse models [16, 31]

Under selection pressure with CBA, susceptible viruses may evolve towards resistance through loss of key N-linked glycosylation sites [25, 185-188]. Interestingly, in the case of HIV-1, CBA resistance correlates with reduced viral fitness and enhanced susceptibility to neutralizing antisera [14, 186, 188]. Consequently, it has been suggested that CBA therapy of chronic viral infections like HIV-1 and HCV may promote viral evolution towards resistance to the CBA concomitant with enhanced susceptibility to host immune control. This concept of CBA-mediated immunotherapy holds considerable appeal as a method to promote durable immune control, and perhaps even eradication of HIV infection [14]. Given GRFT's antiviral activity in the mid picomolar range, and our data here that show that the drug accumulates to relevant therapeutic concentrations which are tolerated with minimal toxicity, GRFT is a strong candidate for further experimental testing of this idea first put forward by Dr. Balzarini and colleagues [14]. 

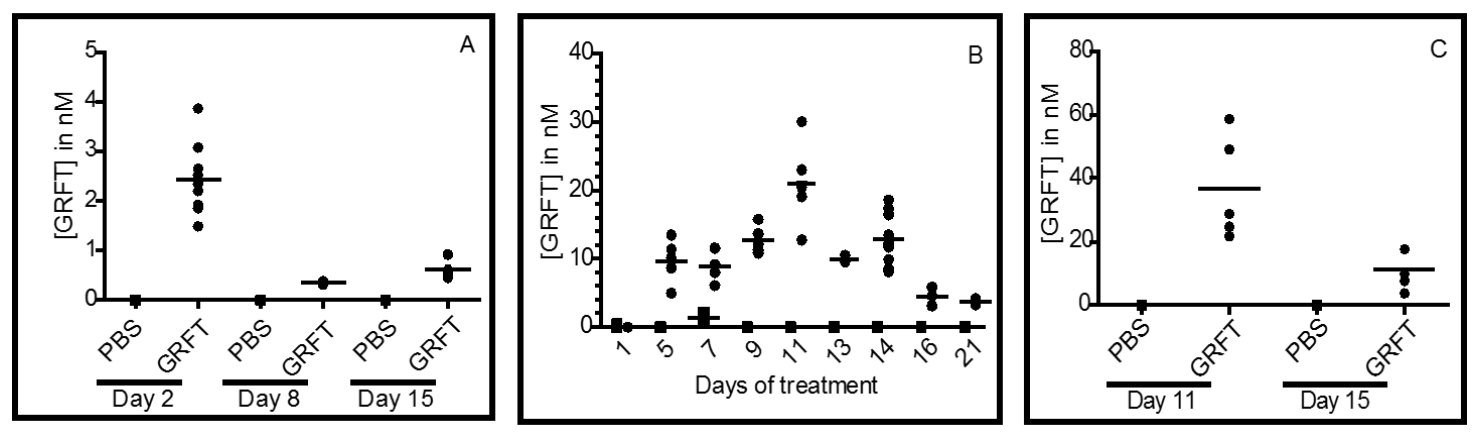

Figure 2.1 Griffithsin concentrations in plasma and serum samples. Data were obtained from mouse plasma after a single high-dose administration of $50 \mathrm{mg} / \mathrm{kg}$ GRFT (A), a chronic daily administration of $10 \mathrm{mg} / \mathrm{kg} \mathrm{GRFT} \mathrm{(B),} \mathrm{and} \mathrm{from} \mathrm{guinea}$ pig serum after 10 daily administrations of $10 \mathrm{mg} / \mathrm{kg}$ GRFT (C). PBS was administered to control animals and bars indicate mean group concentration. 

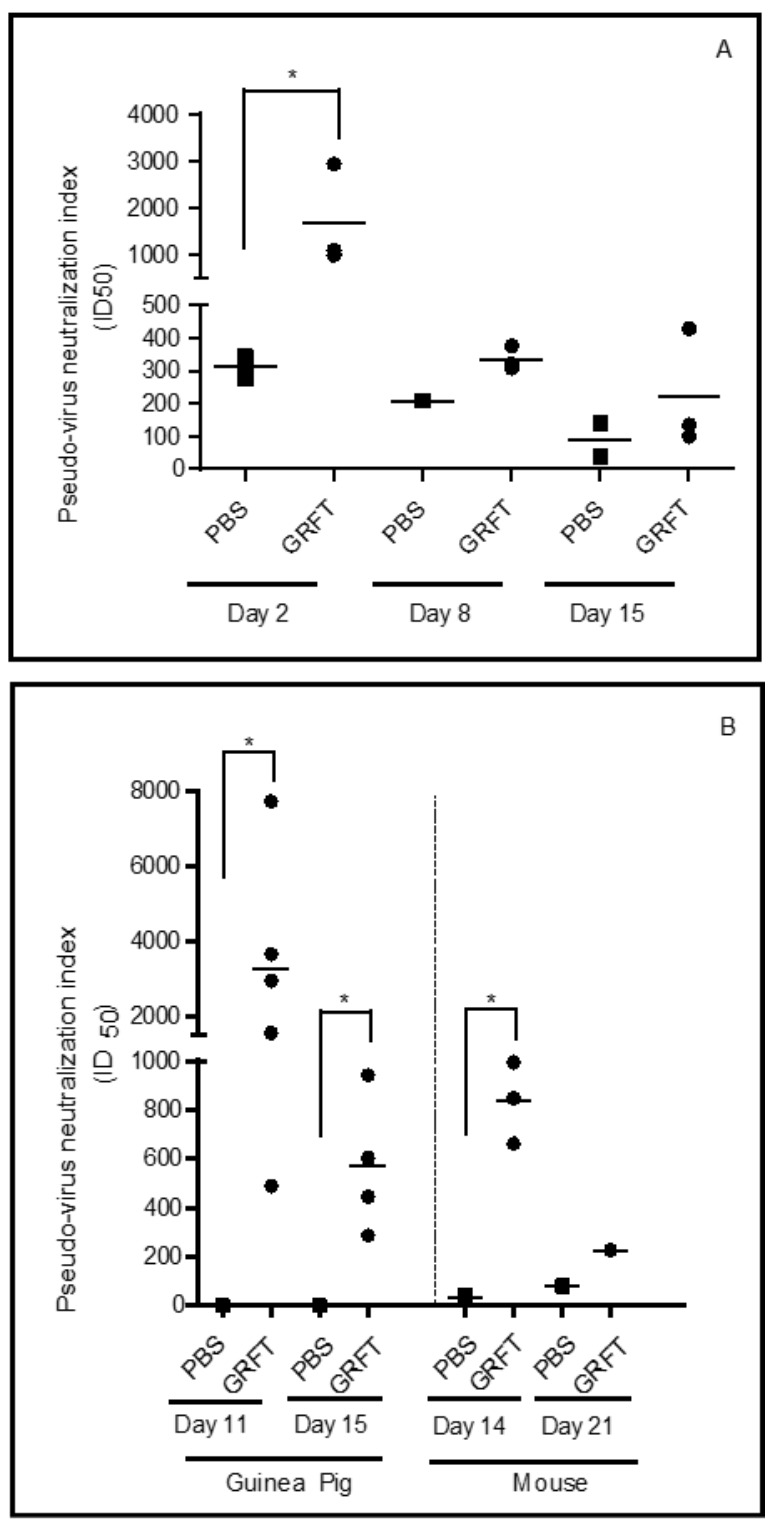

Figure 2.2. Antiviral activity of samples collected from GRFT treated animals.

HIV-1 env- pseudovirus neutralization activity was assessed for mouse plasma after a single high dose administration of $50 \mathrm{mg} / \mathrm{kg}$ GRFT (A) and expressed as ID50. The ID50 values were obtained for samples from guinea pigs and mice chronically treated with $10 \mathrm{mg} / \mathrm{kg}$ GRFT (B). Control animals were treated with PBS bars indicate mean group concentration. 1-way ANOVA significance $(p<0.05)$ is indicated by $\left(^{*}\right)$. 


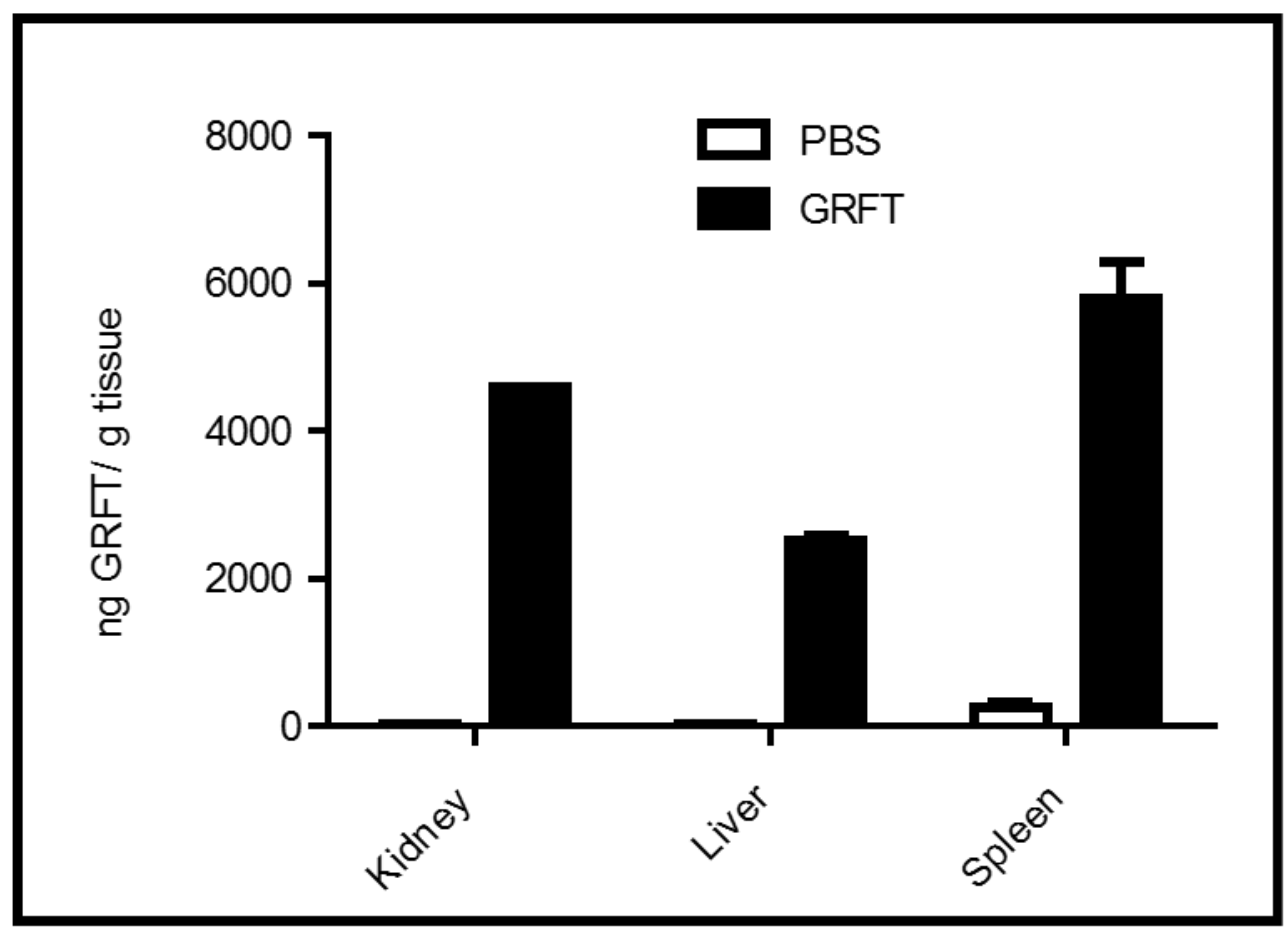

Figure 2.3. Quantitation of GRFT in mouse organs. Pooled Protein samples were extracted from kidneys, livers, and spleens after a chronic subcutaneous administration of GRFT or PBS and GRFT was detected using a gp120-binding ELISA. 


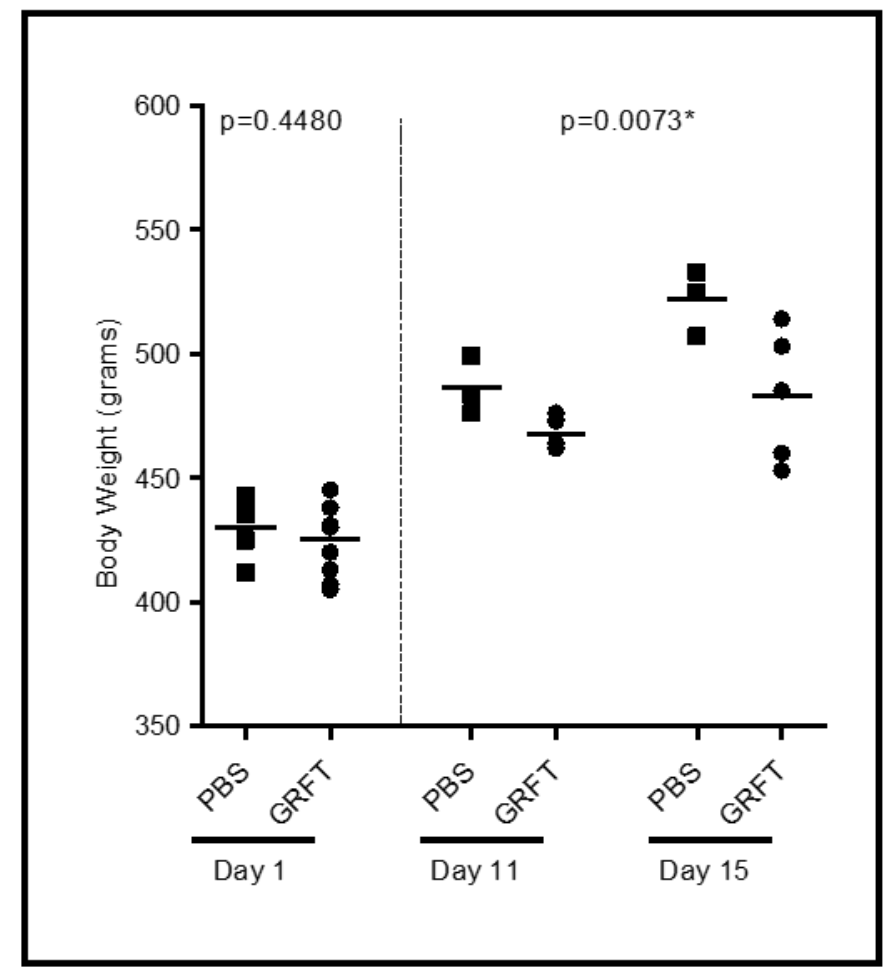

Figure 2.4. Guinea pig body weight gain as an indicator of overall health. Body weights were measured on experimental Day 1 and on the termination day (Day 11 or Day 15$)$. Significance $(p<0.05)$ is indicated by $\left(^{*}\right)$. 

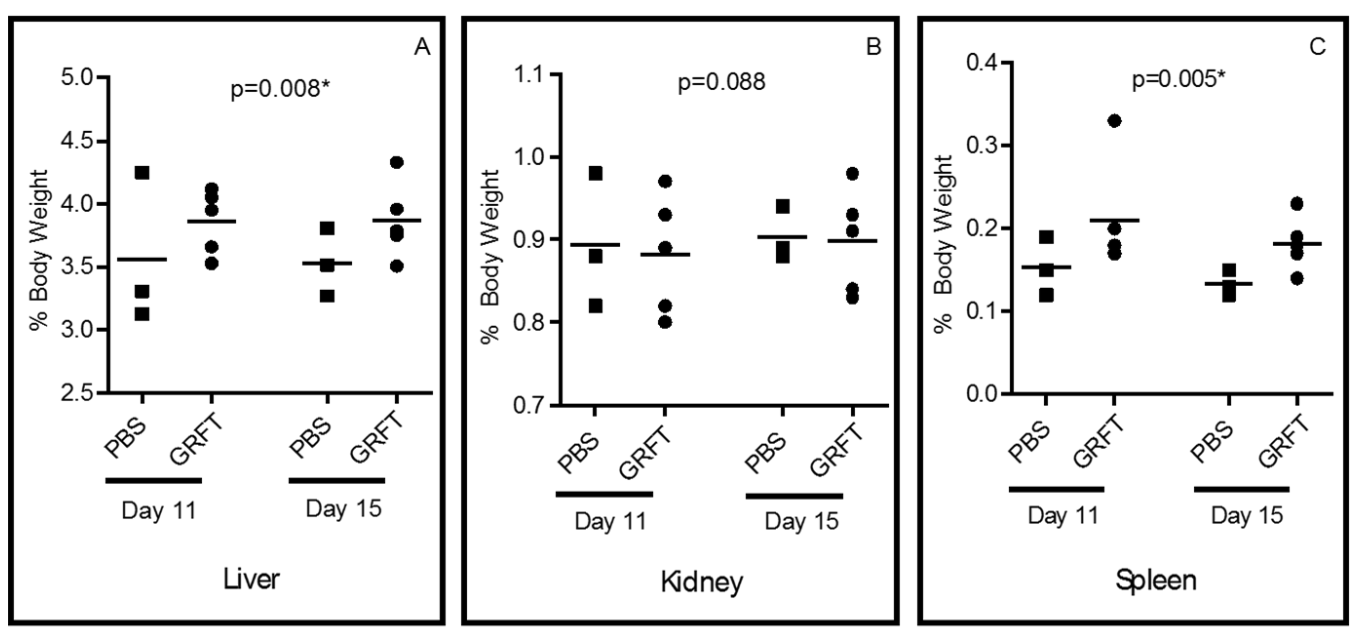

Figure 2.5. GRFT does not affect guinea pig organ weights. Liver (A), kidney (B), and spleens (C) weights were measured relatively to body weight at sacrifice. 


\begin{tabular}{ccccccc} 
& & & \multicolumn{2}{c}{ Day 11} & \multicolumn{2}{c}{ Day 15 } \\
\hline Cell Type & Parameter & Unit & PBS $(\mathrm{n}=3)$ & GRFT $(\mathrm{n}=5)$ & PBS $(\mathrm{n}=3)$ & GRF $(\mathrm{n}=5)$ \\
\hline Leukocyte & & & & & & \\
& $\mathrm{WBC}$ & $\mathrm{k} / \mu 1$ & $4.92 \pm 2.43$ & $2.15 \pm 0.23$ & $4.35 \pm 1.28$ & $2.90 \pm 1.36$ \\
& $\mathrm{NE}$ & $\mathrm{k} / \mu 1$ & $2.30 \pm 1.46$ & $0.96 \pm 0.13$ & $1.67 \pm 0.45$ & $1.01 \pm 0.42$ \\
& $\mathrm{LY}$ & $\mathrm{k} / \mu 1$ & $2.49 \pm 1.11$ & $1.14 \pm 0.28$ & $2.60 \pm 0.89$ & $1.82 \pm 0.99$ \\
& $\mathrm{MO}$ & $\mathrm{k} / \mu 1$ & $0.09 \pm 0.10$ & $0.03 \pm 0.03$ & $0.07 \pm 0.03$ & $0.06 \pm 0.04$ \\
& $\mathrm{EO}$ & $\mathrm{k} / \mu 1$ & $0.03 \pm 0.02$ & $0.02 \pm 0.01$ & $0.02 \pm 0.01$ & $0.01 \pm 0.02$ \\
& $\mathrm{BA}$ & $\mathrm{k} / \mu 1$ & $0.00 \pm 0.01$ & $0.00 \pm 0.00$ & $0.00 \pm 0.00$ & $0.00 \pm 0.00$ \\
\hline Erythrocyte & & & & & & \\
& $\mathrm{RBC}$ & $\mathrm{M} / \mu 1$ & $4.30 \pm 0.06$ & $4.12 \pm 0.18$ & $4.75 \pm 0.21$ & $4.37 \pm 0.22$ \\
& $\mathrm{Hb}$ & $\mathrm{g} / \mathrm{dl}$ & $12.23 \pm 0.64$ & $11.64 \pm 0.46$ & $13.10 \pm 0.46$ & $12.22 \pm 0.61$ \\
& $\mathrm{HCT}$ & $\%$ & $38.30 \pm 2.31$ & $36.12 \pm 1.47$ & $41.57 \pm 1.76$ & $39.52 \pm 1.94$ \\
& $\mathrm{MCV}$ & $\mathrm{FL}$ & $88.97 \pm 4.73$ & $87.58 \pm 1.29$ & $87.53 \pm 1.70$ & $90.40 \pm 0.52^{*}$ \\
& $\mathrm{MCH}$ & $\mathrm{pg}$ & $28.43 \pm 1.33$ & $28.26 \pm 1.52$ & $27.63 \pm 0.61$ & $27.96 \pm 1.04$ \\
& $\mathrm{MCHC}$ & $\mathrm{g} / \mathrm{dl}$ & $31.97 \pm 0.25$ & $32.26 \pm 1.33$ & $31.50 \pm 0.26$ & $30.94 \pm 1.11$ \\
& $\mathrm{RDW}$ & $\%$ & $13.83 \pm 1.43$ & $12.92 \pm 0.73$ & $14.13 \pm 0.29$ & $12.66 \pm 0.70^{*}$ \\
\hline Thrombocyte & & & & & & \\
& $\mathrm{PLT}$ & $\mathrm{k} / \mu 1$ & $595.33 \pm 54.31$ & $628.60 \pm 44.77$ & $677.33 \pm 11.93$ & $659.00 \pm 34.26$
\end{tabular}

Table 2.1. Hematological profile for guinea pigs after chronic treatment with GRFT. Data represent the mean values +/- standard deviation for white blood cells (WBC), neutrophils (NE), lymphocytes (LY), monocytes (MO), eosinophils (EO), basophils (BA), red blood cells (RBC), hemoglobin $(\mathrm{Hb})$, hematocrit $(\mathrm{HCT})$, mean corpuscular volume (MCV), mean cell hemoglobin $(\mathrm{MCH})$, mean cell hemoglobin concentration $(\mathrm{MCHC})$, red cell distribution width (RDW), platelets $(P L T)$, and mean platelet volume (MPV). Significance $(p<0.05)$ is indicated by $\left({ }^{*}\right)$. 

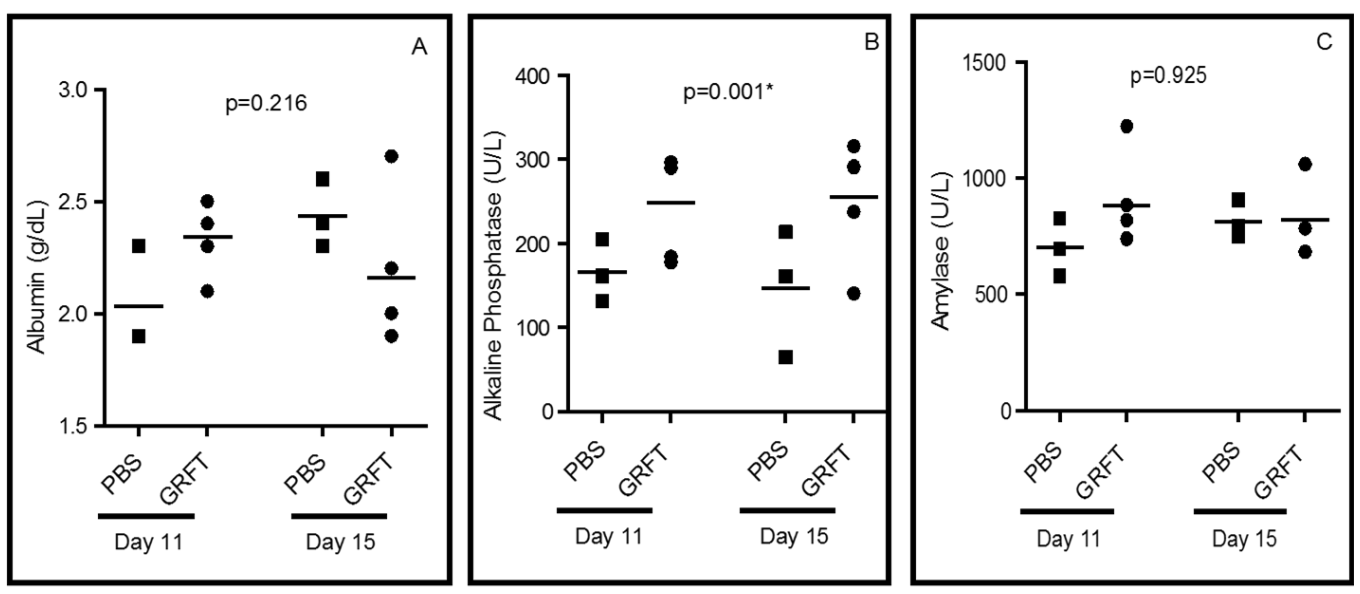

Figure 2.6. Serum chemistry data for guinea pigs after chronic treatment with GRFT. Levels of Serum Albumin (A), Alkaline Phosphatase (B), and Amylase (C) were obtained from GRFT or PBS treated animals at sacrifice. Bars indicate mean group concentration. 


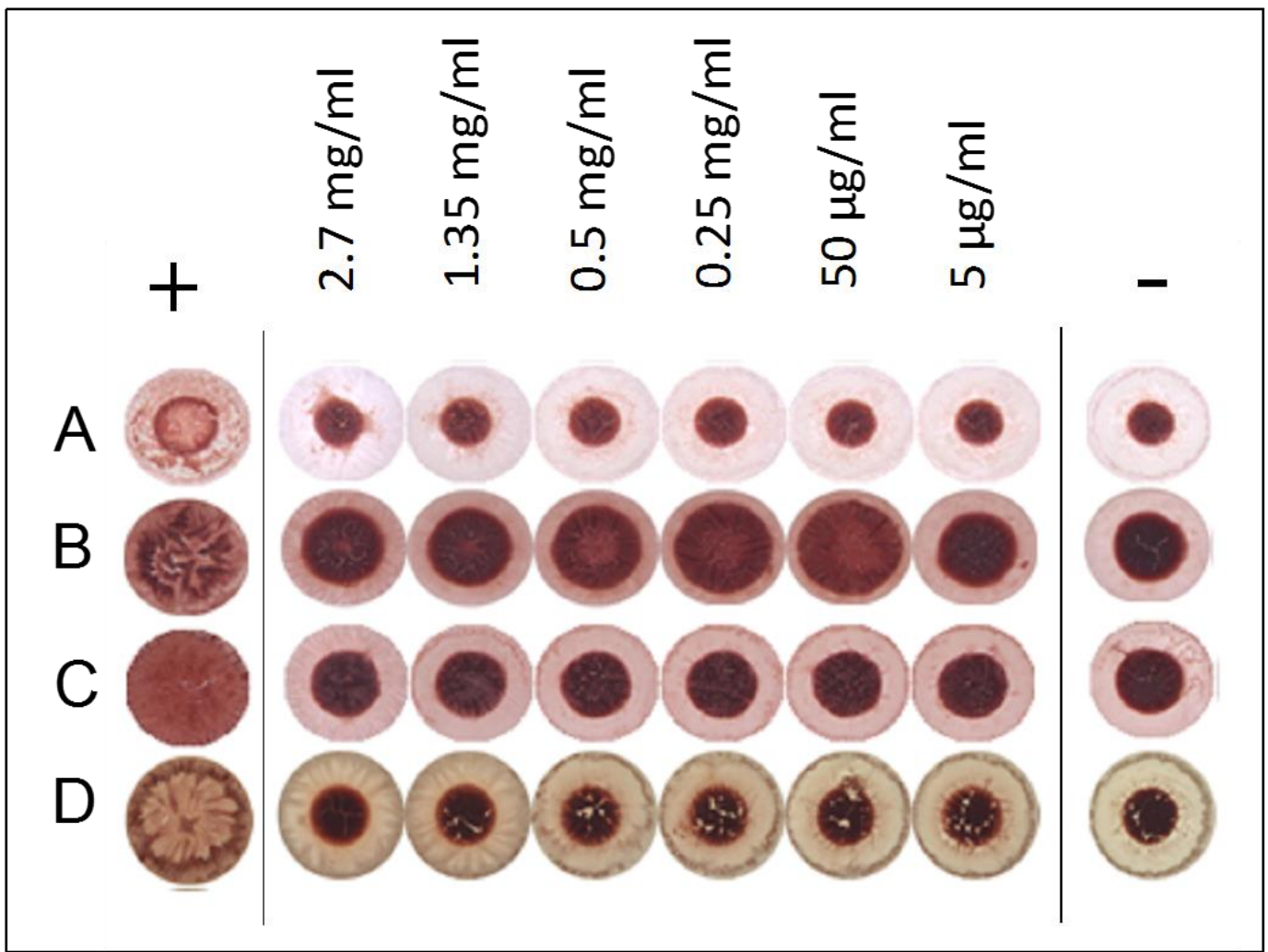

Figure 2.7. Hemaggluttination activity of GRFT. A wide range of GRFT concentrations ( $5 \mu \mathrm{g} / \mathrm{ml}$ equals $391 \mathrm{nM}$ ) was used to examine GRFT's potential hemagluttination activity on erythrocytes from multiple species including sheep (A), Guinea pig (B), Human (C), and Mouse (D). PHA (5 $\mu \mathrm{g} / \mathrm{ml})$ and PBS were used as positive and negative controls, respectively. 


\section{CHAPTER 3}

\section{THE PHARMACOKINETCIS OF THE ANTIVIRAL LECTING GRIFFITHSIN INDICATED MULTIPLE POTENTIAL USES AND CHALLENGES}

\section{INTRODUCTION}

Various lectins, carbohydrate binding proteins that bind specific glycan structures, are important components of many organisms' innate pathogen defense system. Several lectins isolated from natural sources are known to inhibit microbial and viral infections in vitro and in vivo [29, 31, 37, 142, 189, 190]. Griffithsin, derived from the red alga Griffithsia, is a dimeric lectin with a total of 6 binding sites capable of binding terminal oligomannose residues on asparagine (N)-linked Man5-9GlcNAc2 structures[32, 163-166]. Mannose terminal glycan structures appear to comprise a significantly greater proportion of glycans on pathogen surfaces than in mammalian host cells. In fact, oligomannose structures comprise the vast majority of N-linked glycans in the HIV-1 glycan shield $[13,60]$, where they assist in the virus's evasion of immune surveillance [40]. Multiple studies have described Griffithsin's HIV-1 neutralization $\mathrm{EC}_{50}$ in vitro as being within the mid picomolar range $[16,32,169]$. GRFT also exhibits 
nanomolar activity against several other enveloped viruses such as HCV, HSV-2, SARS CoV, and JEV, amongst others[23, 31, 36, 173]. GRFT prevents viral infection in vitro by binding the envelope oligomannose glycans, and thereby occluding functionally important domains of the viral envelope protein, preventing interactions with cell-surface receptors, or otherwise impeding structural transitions necessary to effect infection. In the case of HSV-2, GRFT may also act by inhibiting virus egress and spread post-infection[36]. Topical application of GRFT prevents HIV-1 infection of human cervical explants [169], and inhibits viral spread in murine HSV-2 vaginal challenge[36].

In preexposure prophylaxis models, GRFT has displayed antiviral activity against HSV-2, SARS-CoV, and HCV [23, 31, 36, 171, 191]. In examining GRFT's systemic chemotherapeutic potential, our previous studies have shown that in two rodent models, GRFT is tolerable in short term subcutaneous dosing, while retaining anti-viral activity in serum [34]. This tolerability profile, retained antiviral activity, and broad spectrum activity against susceptible viruses further supports the study of GRFT as a systemic chemopreventative or chemotherapeutic.

While GRFT has initially been evaluated as a potential topical microbicide, its pharmacokinetic profile - which could limit its practical utility as chemotherapeutic or chemopreventative - is currently unknown. Given GRFT's observed resistance to low $\mathrm{pH}$ and a number of proteases[192], we hypothesized that GRFT may have some degree of oral bioavailability. We compared the concentration of GRFT in serum from treated animals following intravenous, 
subcutaneous, and oral dosing, and report that the pharmacokinetic profile of GRFT can vary greatly depending on method of administration. Additionally, we report a small fraction of oral dosage of GRFT formulated in PBS passes through the digestive tract and retains anti-viral activity in fecal material. Further, we found that after both single dose and 10 day chronic oral administration, GRFT was undetectable in serum and urine, indicating that GRFT is either not systemically absorbed to a detectible degree upon ingestion or inactivated during the absorption process. Accordingly, the method of administration should be considered when researching the usage of GRFT as an orally dosed preexposure prophylactic, or intravenously or subcutaneously-dosed systemic therapeutic or chemopreventative. 


\section{MATERIALS AND METHODS}

\section{Lectin reagents}

Recombinant GRFT produced in Nicotiana benthamiana plants as described previously [169] was purified to $>99 \%$ purity in phosphate buffered saline (PBS), pH 7.4 with less than 0.05 Endotoxin Units (EU) per milligram.

\section{Animal housing and care}

250g Sprague Dawley Rats (Rattus norvegicus, Charles River Laboratories) were housed in a temperature- and humidity-controlled room with an alternating light/dark cycle of $12 \mathrm{~h}$, with standard diet and water ad libitum. All animal procedures were approved by the University of Louisville's Institutional Animal Care and Use Committee.

Pharmacokinetic Study Dosing and Sample Collection

$250 \mathrm{~g}$ Sprague Dawley rats were procured from Charles River with indwelling femoral vein catheter. Rats were weighed and treated with a single dose of either $10 \mathrm{mg} / \mathrm{kg}$ or $20 \mathrm{mg} / \mathrm{kg}$ GRFT. For intravenous treatment, indwelling catheters were checked for patency and either $10 \mathrm{mg} / \mathrm{kg}$ or $20 \mathrm{mg} / \mathrm{kg}$ GRFT was infused into the catheter over a 15 second period. Catheters were then flushed by PBS infusion of 3 times the catheter volume and filled with heparinized glycerol (Braintree Scientific) before reinsertion of the catheter plug. For subcutaneous treatment, $10 \mathrm{mg} / \mathrm{kg}$ or $20 \mathrm{mg} / \mathrm{kg}$ GRFT was injected under the 
skin between shoulders. For oral dosing treatment, $2.5 \mathrm{mg}$ of GRFT formulated to a final volume of $1 \mathrm{ml}$ was dosed via gastric lavage.

In intravenously and subcutaneously dosed animals, approximately $150 \mu \mathrm{l}$ of blood was drawn via indwelling femoral catheter at the following time points after GRFT administration: 15 minutes, 30 minutes, 1 hour, 2 hour, 4 hour, 8 hour, 24 hour, and 48 hours (sacrifice). To capture later time points, an additional cohort of animals had 150ul blood drawn at 72 and 96 hours post dosage. Orally dosed animals had $150 \mu$ of blood drawn at the following time points post administration: 10 minutes, 20 minutes, 30 minutes, 1 hour, 2 hours, 4 hours, 6 hours, 24 hours and 24 hours. All animals were sacrificed by $\mathrm{CO}_{2}$ asphyxiation followed by thoracotomy at the conclusion of the study.

\section{Chronic Oral Dosing Treatment and Sample Collection}

18 rats were treated daily with either $1 \mathrm{ml}$ PBS $(n=6), 5 \mathrm{mg}$ GRFT $(n=6,1$ $\mathrm{ml}$ dosing volume), or $10 \mathrm{mg}$ GRFT ( $\mathrm{n}=6,1 \mathrm{ml}$ dosing volume) for a period of 10 days via gastric lavage. On treatment day 5 , blood samples were drawn via lateral tail vein approximately 3 hours after oral dosing. On day 10 , animals were treated, and approximately 3 hours after treatment, animals were sacrificed via $\mathrm{CO}_{2}$ asphyxiation followed by thoracotomy. Blood was drawn by cardiac puncture after euthanasia. Urine was taken at selected time points after oral dosing. 


\section{Active Mass Balance Rat Treatment and Sample Collection.}

10 rats were treated with a single dose of either $2.5 \mathrm{mg} \mathrm{GRFT}(10 \mathrm{mg} / \mathrm{ml})$ through either intravenous indwelling catheter or subcutaneous dose. 5 rats were treated with $2.5 \mathrm{mg}$ of GRFT (1 $\mathrm{ml}$ dosing volume) via gastric gavage. Post dosage, animals were placed in metabolic cages and urine was collected at the following time points after treatment: 4 hours, 8 hours, 12 hours, 20 hours, and 48 hours. Fecal pellets were collected at 24 and 48 hours. Urine volume and fecal pellet mass was quantitated for mass balance calculation.

Fecal Pellet Collection and Extract preparation - Chronic Oral Treatment

Fecal pellets from treated animals were obtained at 8 and 24 hours after initial treatment and at selected days thereafter by randomly drawing 6 dried pellets from animal cage bedding. Briefly, for fresh fecal pellets not requiring additional PBS for rehydration, $1 \mathrm{ml}$ of PBS was added per $1 \mathrm{gram}$ of pellets in a $1.5 \mathrm{ml}$ Eppendorf tube. For dried fecal pellets requiring rehydration $2 \mathrm{ml}$ of PBS per were added per 1 gram of pellets in an Eppendorf tube. Pellets were allowed to absorb the PBS for 15-30 minutes before crushing and mixing. After crushing and mixing, the resulting fecal slurry was centrifuged at $1000 \mathrm{~g}$ for 5 minutes and supernatant removed. Supernatant was spun again to pellet and remove and debris and final extract supernatant was removed and aliquoted for GRFT concentration determination and anti-HIV activity testing. Fecal extract samples were pooled by combining equal volumes of extract from each cage within the applicable treatment group. 
Fecal pellet extracts from chronically treated animals underwent additional processing to further remove contaminants before HIV-1 pseudovirus neutralization assays. Samples were sterilized and filtered using Costar Spin-X centrifuge Tube Filters with a $0.22 \mu \mathrm{m}$ Cellulose acetate filter. After filtration, samples were then dialyzed in a dialysis cassette with a 2000 molecular weight cut off membrane in PBS for 24 hours at $4^{\circ} \mathrm{C}$.

Extracts for mass balance experiments were prepared by desiccation and rehydration of fecal pellets. After overnight desiccation in a vacuum centrifuge, pellets were pulverized into powder using a commercial coffee grinder. Pellet extracts were formulated using $0.5 \mathrm{~g}$ of dried powder and $2 \mathrm{ml}$ of PBS. After rehydration the resulting fecal slurry was thoroughly mixed and centrifuged at $1000 \mathrm{~g}$ for 5 minutes and supernatant removed. Supernatant was spun again to pellet and remove and debris and final extract supernatant was removed and aliquoted for GRFT concentration determination.

GRFT capture immunoassay using the Influenza $H A$

To detect trace amounts of GRFT present in serum and fecal extracts, we used an ELISA as previously described with modifications [34]. Briefly, Maxisorp plates (Nunc) were coated with $10 \mu \mathrm{g} / \mathrm{ml}$ purified influenza hemagglutinin (HA) in PBS (Kentucky Bioprocessing) and incubated overnight at $4^{\circ} \mathrm{C}$. Plates were blocked with $\sim 2.5 \%(\mathrm{w} / \mathrm{v})$ Bovine Serum Albumin (BSA) in PBS containing 0.05\% Tween 20 (PBS-T). Samples were diluted in blocking buffer and were incubated for 1 hour at RT. Serial dilutions of purified GRFT were run in parallel for 
standard curve generation. Active, HA-bound GRFT was detected by rabbit antiGRFT antiserum (1:25,000) followed by HRP-conjugated goat anti-rabbit IgG $(1: 10,000)$. Plates were developed with SureBlue TMB Microwell Peroxidase Substrate, and reactions were stopped with $1 \mathrm{~N} \mathrm{H}_{2} \mathrm{SO}_{4}$. Absorbance readings were measured at $450 \mathrm{~nm}$ and $570 \mathrm{~nm}$ using a BioTek Synergy HT plate reader.

\section{Evaluation of Anti-HIV Activity of Fecal Extracts.}

HIV-1 neutralization activity of fecal pellet extracts from pooled, chronically treated animals was measured using pseudovirus neutralization assays as previously described [174]. Briefly, DU156 env-pseudotyped virus particles were generated by transfection of 293T cells and titrated in TZM-bl cells. Antiviral activity, a function of luciferase reporter gene activity, was determined by relative luminescence after addition of substrate. $I D_{50}$ values were defined as the sample dilution required to reduce luminescence by $50 \%$ in comparison to wells with no sample added.

\section{Histopathology}

Hematoxylin and eosin stained tissue slides were examined in a blinded manner by a veterinary pathologist (M.P.) for gross or cellular abnormalities.

\section{Pharmacokinetic Analysis}

Pharmacokinetic Parameters were analyzed using PK Solutions Software (Summit PK). Parameters were determined using GRFT serum concentrations at 
sampling time points. Values were generated from time vs. concentration curves based upon amount of GRFT dosed/gram bodyweight. Values generated included Absorption, distribution, and elimination half-lives, area under the curve $(\mathrm{AUC})$, volume of distribution $(\mathrm{Vd})$, clearance, and maximum serum concentration $\left(\mathrm{C}_{\max }\right)$. 


\section{RESULTS}

Pharmacokinetics-Intravenous Dosing

GRFT was detectible at all time points tested - including up to 96 hours post dose (Figure 3.1). 15 minutes post intravenous administration, animals displayed an average serum concentration of approximately $74 \mu \mathrm{g} / \mathrm{ml}$ and 141 $\mu \mathrm{g} / \mathrm{ml}$ based on initial doses of $10 \mathrm{mg} / \mathrm{kg}$ and $20 \mathrm{mg} / \mathrm{kg}$ respectively. By 8 hours after administration, serum concentrations had decreased to average concentrations $897 \mathrm{ng} / \mathrm{ml}$ (10 mg/kg dosage) and $1540 \mathrm{ng} / \mathrm{ml}$ (20 mg/kg dosage) (Figure 3.1A). Analysis of elimination curves by Pk Solutions software revealed a multiphasic elimination, with 3 distinct phases observed. Early phase elimination kinetics (0-2 hrs) revealed an absorption half-life of approximately 0.5 hours, with late phase elimination half-life ranging from 10 to 17 hours (Table 3.1). GRFT remained detectible in serum at low $\mathrm{ng} / \mathrm{ml}$ concentrations up to 96 hours post intravenous treatment (Figure 3.1B).

Pharmacokinetics - Subcutaneous Dosing

After subcutaneous administration, GRFT displayed increasing serum concentrations, reaching peak serum concentration approximately 4 hours post subcutaneous administration (Figure 3.1A). Thereafter GRFT concentrations decreased at rates analogous to those seen in intravenously dosed animals. At $10 \mathrm{mg} / \mathrm{ml}$, GRFT displayed a $C_{\max }$ of $6.6 \mu \mathrm{g} / \mathrm{ml}$ whereas at $20 \mathrm{mg} / \mathrm{ml}$ GRFT displayed a mean $\mathrm{C}_{\max }$ of $19.7 \mu \mathrm{g} / \mathrm{ml}$. GRFT remained detectible in serum at low $\mathrm{ng} / \mathrm{ml}$ concentrations up to 96 hours post subcutaneous treatment. 


\section{Pharmacokinetics - Oral Dosing}

GRFT did not display any detectible serum concentrations at any time point tested after oral administration.

Chronic GRFT oral treatment

Serum collected from rats orally dosed daily with PBS, $5 \mathrm{mg}$ GRFT and 10mg GRFT did not display detectible GRFT concentrations on either day 5 or day 10. Urine samples obtained from these rats also failed to display detectible GRFT concentrations.

Fecal GRFT concentrations -Chronic Dosing

Fecal extracts from fresh fecal pellets displayed low concentrations of GRFT. At 8 hours after initial dosing, animals treated with $5 \mathrm{mg}$ and $10 \mathrm{mg}$ GRFT displayed an average fecal GRFT extract concentration of approximately 2 nM (Figure 3.2A). However, average GRFT concentration of both groups decreased at $24 \mathrm{hr}$ after dosing, with fecal extracts from animals treated with $5 \mathrm{mg}$ GRFT displaying an average concentration of $0.5 \mathrm{nM}$ while animals treated with $10 \mathrm{mg}$ GRFT displayed an average concentration of $0.75 \mathrm{nM}$. (Figure 3.2B). Fecal extracts from random pellet draws also displayed varying amounts of GRFT. Dessicated fecal pellet extracts drawn on days 2, 3, 8 and 9 were analyzed. As expected, none of the fecal pellet extracts from PBS treated animals displayed GRFT concentrations (Figure 3.2C). Fecal pellet extracts from 
animals treated with $5 \mathrm{mg}$ GRFT ranged from $1.7 \mathrm{nM}$ to $20 \mathrm{nM}$. Fecal pellet extracts from animals treated with $10 \mathrm{mg}$ GRFT ranged from $1.7 \mathrm{nM}$ to $126 \mathrm{nM}$.

HIV-1 Neutralization activity Fecal Pellect Extracts of Chronically treated animals.

Pooled fecal extracts from rats treated with $5 \mathrm{mg}$ and $10 \mathrm{mg}$ GRFT neutralized HIV-1 pseudoviruses (Clade C primary sexually transmitted isolate Du156) with an $I D_{50}$ of 590 on experimental day 3 for those animals treated with 5mg GRFT and an ID 50 of 2059 for animals treated with 10 mg GRFT (Figure 3.3). This neutralization activity was also observed in samples obtained at later time points. On treatment day 8 sera from animals receiving a dosage of $5 \mathrm{mg}$ GRFT had an $I_{50}$ of 885 while those receiving $10 \mathrm{mg}$ had an $I_{50}$ of 9452 (Figure 3.3). On treatment day 9 fecal pellet extracts had $I D_{50} S$ of 2195 and 13307 for groups receiving $5 \mathrm{mg} / \mathrm{kg}$ and $10 \mathrm{mg} / \mathrm{kg}$ GRFT respectively (Figure 3.3).

\section{Mass Balance of Active GRFT}

Active concentrations of GRFT were detected in urine or fecal material produced during the first 24 hours following intravenous, subcutaneous and oral dosage of $2.5 \mathrm{mg}$ GRFT. After intravenous administration and subcutaneous administration, GRFT was detected in urine (Figure 3.4). After intravenous administration of $2.5 \mathrm{mg}$ GRFT, approximately $131 \mu \mathrm{g}$ of active GRFT was recovered, whereas $41 \mu \mathrm{g}$ of active GRFT was recovered from subcutaneously treated animals. However, a different elimination profile was observed following oral administration. Following oral administration, approximately $2.3 \mu \mathrm{g}$ GRFT 
was recovered from fecal material. GRFT was not detectible in urine at any time point tested in orally dosed animals.

Histology

Organ slides did not reveal any treatment-related pathologies. However individual variation in slides attributable to sample preparation and sectioning were observed. 


\section{DISCUSSION}

The pharmacokinetics data we derived here expand upon our previous studies (Barton et al.), which showed that subcutaneous treatment with $10 \mathrm{mg} / \mathrm{kg}$ GRFT was well tolerated in two laboratory rodent species (mice and guinea pigs), and accumulated to therapeutically relevant levels - above GRFT's antiHIV EC $E_{50}$ of $40 \mathrm{pM}$. Here we show in rats, a standard rodent model used for PK studies, that GRFT has a complex PK profile which may prove important in designing future studies.

As seen in Figure 3.1, GRFT displays different drug concentration curves depending upon method of administration. Intravenously-dosed GRFT exhibits a multiphasic elimination pattern, with the serum half-life changing over the observation period. While the majority of a single dose of GRFT is eliminated within 8 hours of treatment, potentially therapeutic concentrations above GRFT's anti-HIV-1 $\mathrm{EC}_{50}$ remain for at least 96 hours post administration. This complex elimination pattern, which can vary among other protein based pharmaceuticals in time to maximum serum concentrations and rates of elimination [193-195], may represent multiple processes going on simultaneously. These may include continual absorption, degradation, protein binding, and elimination processes. GRFT was observed to be renally excreted in urine in substantial quantities shortly after dosing intravenous dosing. However, this amount was also observed to decrease over time. Further, prior research within our lab has detected GRFT in vaginal mucous after subcutaneous dosing (observational studies). Thus, it is possible that GRFT could still be distributing more 
extensively into deeper physiological compartments while other fractions of GRFT are being eliminated. This speculation will require in vivo radiolabeling studies of GRFT for a full determination as to GRFT's ultimate fate. Further, it is also possible that degradative proteolysis is occurring. While previous studies have shown GRFT is relatively proteolytically resistant to many proteases, it has been reported that GRFT is susceptible to leukocyte elastase [192]. Thus it is possible that GRFT is vulnerable to some other unknown proteases as well. This possibility will require both extensive in vitro protease degradation testing as well as examination of degradation products in serum after systemic administration using radiolabeled GRFT to determine whether GRFT is being degraded or retained within the system.

The final phase of elimination of GRFT appears to be substantially longer than the initial elimination phases. This persistence is comparable to other protein therapeutics, such as monoclonal antibodies, which also present long serum persistence[194] as well as smaller molecule biologics, such as recombinant epoetin[193]—-though recombinant epoetin concentrations have been observed to be eliminated within two to three days after intravenous dosing [188]. This serum persistence could potentially be from redistribution of GRFT from deeper physiological compartments and mucosal surfaces. However, it is also very possible, if not likely, that these low residual concentrations of GRFT are interacting with or binding to endogenous proteins. To confirm this possibility, in vivo testing with radiolabeled GRFT is suggested to identify if GRFT is localizing to or redistributing from, a particular compartment. Additionally, 
cellular protein binding experiments may be useful to identify membrane bound protein binding partners which may be responsible for GRFT's long serum persistence.

Subcutaneously dosed GRFT reaches peak serum concentrations at approximately 4 hours post administration, with elimination patterns mirroring intravenous dosage thereafter. The initial distribution phase immediately following subcutaneously dosage appeared to be fairly comparable regardless of amount of GRFT dosed. However, subcutaneous doses at higher drug concentration increased GRFT's peak concentration, total drug exposure and bioavailability, as reflected in the area under the curve over the study period. It is thought that the approximately 4 fold difference in total drug exposure observed between $10 \mathrm{mg} / \mathrm{kg}$ and $20 \mathrm{mg} / \mathrm{kg}$ subcutaneous doses of GRFT could be attributable to a gradual release or absorption of more GRFT from the site of subcutaneous administration over the time course of treatment while competing distributive, elimination, and degradative processes are occurring as it enters the circulatory system from the capillary bed. Interestingly, it appears that the concentration of GRFT dosed, and not necessarily the volume of the subcutaneous bolus affected total drug exposure over the study period. A large volume, lower concentration of GRFT tested did not have the sustained higher serum concentrations (data not shown).

These pharmacokinetic results would seem to indicate that in a postexposure prophylactic setting, subcutaneous administration of GRFT may be a viable method of administration. In the cases of Ebola virus and SARS-CoV, the 
viral latency period can range from 2 days or longer. In the context of HIV-1 exposure clinical settings, post-exposure prophylaxis initiated within $24-48$ hours of exposure has shown a protective effect [120]. Additionally, there are now anecdotal evidence that even following seroconversion, immediate treatment with anti-viral regimens can confer clinical benefits [196-198], and in the case of an infant treated after birth, functional cure [199, 200]. Accordingly GRFT's observed pharmacokinetic profile, peak serum concentrations achieved 4 hours post dosing, and persistence up to 96 hours post single treatment would suggest the viability of daily subcutaneous self-dosage regimen to maintain potentially therapeutic effect.

GRFT's pharmacokinetic parameters will also be important in future studies. Given that subcutaneous dosing may be a viable regimen to maintain potentially therapeutic drug concentrations, the pharmacokinetic parameters of GRFT may be useful in study design parameters such as: steady state drug concentrations, loading doses, maintenance doses, and dosing intervals. GRFT's $\mathrm{EC}_{50}$ for HIV is approximately $40 \mathrm{pM}$ [32]. From the clearance parameters $(0.06 \mathrm{~L} / \mathrm{hr})$ determined for $10 \mathrm{mg} / \mathrm{kg}$ subcutaneous dosing, a 72 hour dosing regimen of $2.5 \mathrm{mg}$ GRFT would achieve a stead state concentration of 47 nM, over 1000 times higher than GRFT's $\mathrm{EC}_{50}$ for HIV-1. However, GRFT'sHCV $\mathrm{EC}_{50}$ has been described as $14 \mathrm{nM}$ [31], whereas its SARS-CoV $\mathrm{EC}_{50}$ is approximately $48 \mathrm{nM}$ [23]. Accordingly, as GRFT is further studied in in vivo animal models for varying viral diseases, it may be necessary to design dosing intervals and concentrations necessary to maintain serum drug concentrations at 
potentially therapeutic steady state levels for that disease, while avoiding concentrations that may induce toxicities. For example, with a calculated 14 hour elimination-phase half-life seen in a $10 \mathrm{mg} / \mathrm{kg}$ subcutaneous dose and a $43 \%$ bioavailability, a 72-hour dosing regimen would be necessary to minimize bioaccumulation of GRFT. This data can also be utilized to design a more frequent dosing regimen to minimize peak and trough concentrations of the drug while maintaining a steady state serum concentration above concentrations necessary for potentially therapeutic effect. For instance, to maintain a steady state concentration of $47 \mathrm{nM}, 35 \mu \mathrm{g} / \mathrm{hr}$ or $840 \mu \mathrm{g} /$ day would need to be administered. Alternatively, using these data, a loading dose followed by regular maintenance dosing may be employed to achieve a desired steady state concentrations above the $\mathrm{EC}_{50}$ for particular disease study model.

Interestingly, it also appears that the concentration of subcutaneously dosed GRFT can affect its bioavailability. At 10mg/kg dosing, GRFT displayed a $43 \%$ bioavailability when subcutaneously dosed. Whereas at $20 \mathrm{mg} / \mathrm{kg}$ dosing, GRFT displayed a $90 \%$ bioavailablity (calculated as AUC(subcutaneous)/AUC(intravenous) for each respective dose concentration). The cause of this discrepancy is currently unclear and will require further study. However, it highlights the impact that selection of dose concentration/kg may have upon study design.

In contrast to subcutaneous and intravenous routes, orally administered GRFT does not appear to be systemically absorbed. Other lectins, such as wheat germ agglutinin, which bind to N-acetyl-D-glucosamine and sialic acid 
structures, have been used to increase systemic absorption of some other drugs [201, 202]. However, given GRFT's unique affinity for oligomannose glycans, it was uncertain whether GRFT would have a similar utility. At no time point examined was GRFT found in the serum of orally-treated animals. While this difference in absorption and elimination profile likely makes GRFT unsuitable as an orally dosed systemic therapeutic at this time, advances in drug delivery systems for oral dosing of biologics may eventually enable an oral dosing method for GRFT. However, GRFT's current oral dosing profile raises the potential of GRFT to be an orally-dosed, rectal microbicide for prevention of transmission of HIV-1 and other sexually transmitted pathogens that GRFT is active against (such as HSV-2). Non-absorption of GRFT via oral administration allowed for a fraction of the drug to pass through the digestive tract, ultimately being expelled in fecal waste (Figure 3.4). While this fraction is small, approximately $1 \%$ of the initial dosage, methods to shield GRFT from further effects of stomach acids and proteases could possibly be employed to make this fraction higher. Additionally, as food was not controlled prior to oral administration of GRFT, food consumption could potentially impact GRFT's intestinal transit and successful proteolytic survival. Nonetheless, as seen in both the single oral dose mass balance as well as the chronic oral dosing, the concentration of GRFT in prepared fecal extracts exceed GRFT's $\mathrm{EC}_{50}$ versus HIV-1 over the first 24 hours post treatment. Interestingly however, GRFT's neutralization activity in fecal extract did not correlate well with GRFT's previously described $\mathrm{EC}_{50}$ of $40 \mathrm{pM}$, though neutralization activity did correlate well with GRFT concentration. It is 
believed that this discrepancy may be attributable to the background neutralization activity seen in fecal extracts from PBS treated animals.

Daily prophylactic treatment for HIV-1 is well established. For example, Truvada is currently being prescribed to high risk populations, namely MSM to prevent HIV infection [106, 203]. Additionally, anti-retrovirals are employed following occupational and non-occupational exposure to prevent HIV infection [117-120, 204]. Thus, the observed fractional survival of active GRFT raises the potential that GRFT could be utilized as a daily dosed treatment to "seed" the bowels and rectal mucosa, where it could potentially block HIV infection through its interaction with high mannose glycans on free virions.

Interestingly, the timing of passage of GRFT following oral dosage also appears to be consistent with typical intestinal transit time of 20-30 hours. As seen in Figure 3.2, some animals chronically treated GRFT began to excrete GRFT in their fecal matter within 8 hours of initial treatment regardless of dosage size. At 24 hours after treatment, more than half of the animals from each treatment group were still excreting GRFT in their feces. With an intestinal transit time of 8-24 hours, it would appear that the majority of GRFT administered is passed between 8-20 hours of dosage in rats. However, it is currently unknown what effect the co-administration of food would have upon GRFT's transit time as food was only restricted 20 minutes before administration. It is very possible that intestinal transit time for GRFT would be increased if food were restricted before administration. 
While enveloped viruses such as SARS-CoV, HIV-1, HSV-2 and HCV continue to spread worldwide, it is imperative that all avenues for prevention be evaluated to assess their overall population level effect. Our findings indicate that GRFT's pharmacokinetic profile supports multiple potential uses as well as enables the design of different dosing regimens. As a systemic post-exposure therapeutic or treatment, subcutaneous self-dosing would be both a viable and effective means to achieve potentially therapeutic serum concentrations of GRFT. In the context of post-exposure prophylaxis to emerging viral threats, an initial intravenous bolus treatment may be useful to achieve very rapid peak serum levels of GRFT (Figure 3.1.) Further, given the apparent non-absorption of GRFT following oral administration, as well as the continued anti-viral activity of GRFT found in fecal materials, it is possible that GRFT could be utilized as an orally dosed rectal microbicide in a pre-exposure prophylactic setting. While admittedly, it is only a small fraction of GRFT dosage which survives intestinal transit, we have demonstrated that levels above its anti-HIV EC $\mathrm{E}_{50}$ are achievable. Nonetheless, methods may be needed to shield GRFT from excessive degradation or inactivation. 


\section{GRFT Serum Concentration}
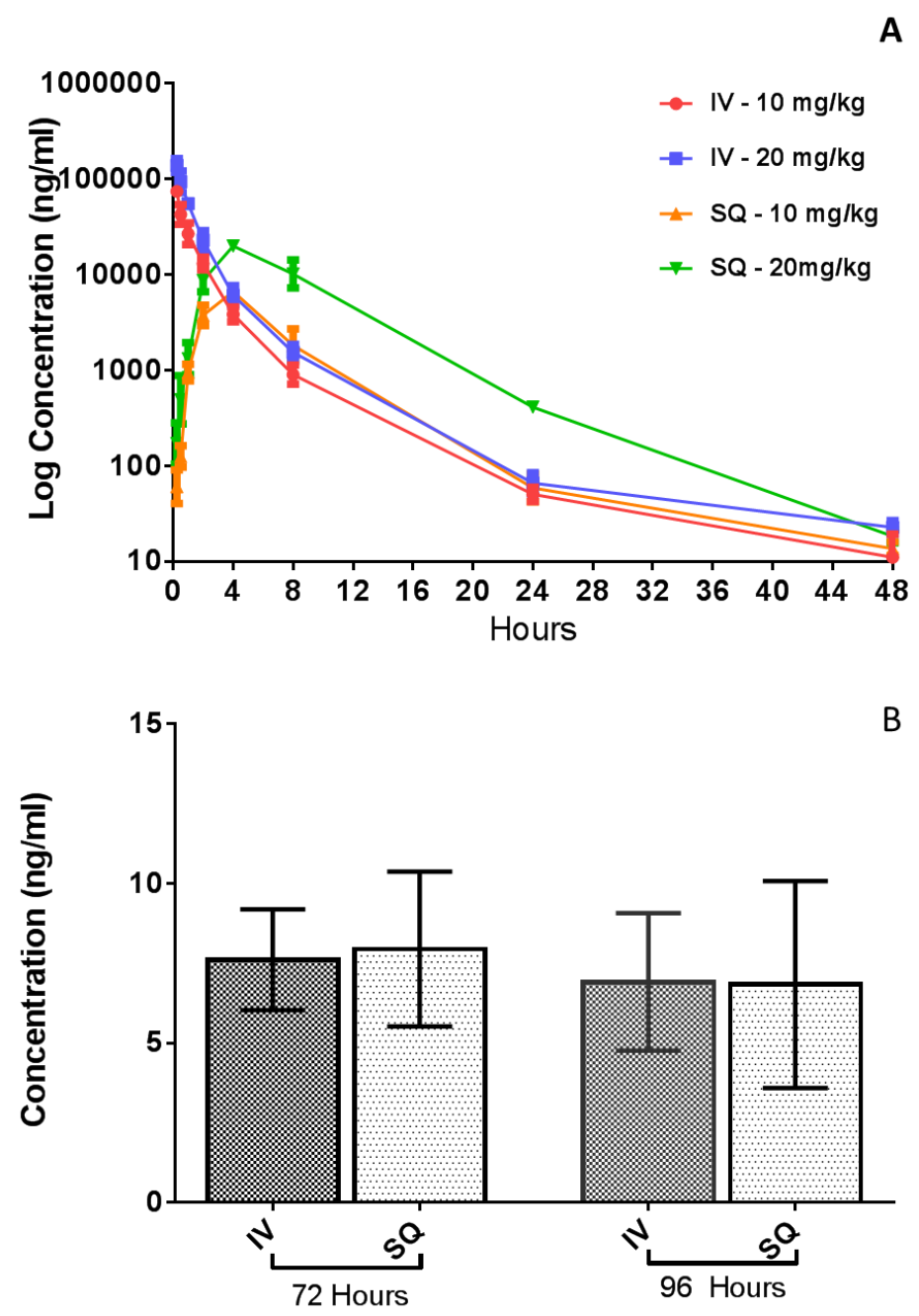

Figure 3.1. Pharmacokinetic profile of GRFT in Serum. Mean GRFT

concentrations present after single Intravenous (IV) and Subcutaneous (SQ) dose ( $n=3-4$ per group) (A). Serum Concentrations from animals dosed with 10 $\mathrm{mg} / \mathrm{kg}$ GRFT both 72 and 96 hours after administration (B). Bars indicate mean \pm standard deviation of 5 biological replicates 


\begin{tabular}{|c|c|c|c|c|c|}
\hline & & \multicolumn{2}{|c|}{ INTRAVENOUS } & \multicolumn{2}{|c|}{ SUBCUTANEOUS } \\
\hline & Units & $\begin{array}{c}10 \mathrm{mg} / \mathrm{kg} \\
(\mathrm{N}=4)\end{array}$ & $\begin{array}{c}20 \mathrm{mg} / \mathrm{kg} \\
(\mathrm{n}=3)\end{array}$ & $\begin{array}{c}10 \mathrm{mg} / \mathrm{kg} \\
(\mathrm{n}=4)\end{array}$ & $20 \mathrm{mg} / \mathrm{kg}(\mathrm{n}=4)$ \\
\hline $\begin{array}{l}\text { INITIAL } \\
\text { DISTRIBUTION } \\
\text { HALF LIFE } \\
\text { (0.25-2 HR) }\end{array}$ & $\mathrm{hr}$ & $0.51 \pm 0.09$ & $0.46 \pm 0.18$ & -- & -- \\
\hline $\begin{array}{l}\text { ABSORPTION } \\
\text { HALF LIFE } \\
\text { (0.25-2 HR) }\end{array}$ & $\mathrm{hr}$ & -- & -- & $1.3 \pm 0.3$ & $1.6 \pm 0.4$ \\
\hline $\begin{array}{l}\text { DISTRIBUTION } \\
\text { HALF LIFE } \\
\text { (4-8 HR) }\end{array}$ & $\mathrm{hr}$ & $1.7 \pm 0.3$ & $2.1 \pm 0.7$ & $2.1 \pm 0.9$ & $2.8 \pm 1.2$ \\
\hline $\begin{array}{l}\text { ELIMINATION } \\
\text { HALF LIFE } \\
\text { (24-48 HR) }\end{array}$ & $\mathrm{hr}$ & $10.7 \pm 4.6$ & $17.5 \pm 6.1$ & $13.8 \pm 6.8$ & $6.6 \pm 1.9$ \\
\hline AUC & $\mathrm{mg}-\mathrm{hr} / \mathrm{L}$ & $110 \pm 20$ & $200 \pm 30$ & $50 \pm 10$ & $180 \pm 50$ \\
\hline VD & $\mathrm{L}$ & $0.35 \pm 0.13$ & $0.57 \pm 0.15$ & $1.2 \pm 0.6$ & $0.23 \pm 0.10$ \\
\hline CLEARANCE & $\mathrm{L} / \mathrm{hr}$ & $0.026 \pm 0.006$ & $0.023 \pm 0.004$ & $0.058 \pm 0.008$ & $0.024 \pm 0.006$ \\
\hline$C_{\operatorname{MAX}}$ & $\mu \mathrm{g} / \mathrm{ml}$ & $80 \pm 30$ & $180 \pm 30$ & $6.6 \pm 0.6$ & $20 \pm 2$ \\
\hline
\end{tabular}

Table 3.1. Pharmacokinetic parameters of GRFT over 48 hours. Data represent the mean values $+/$ - standard deviation for initial distribution (intravenous), absorption (subcutaneous), distribution, and elimination half-lives, area under the curve (AUC), volume of distribution (VD), clearance, and maximum serum concentration $\left(\mathrm{C}_{\max }\right)$. 

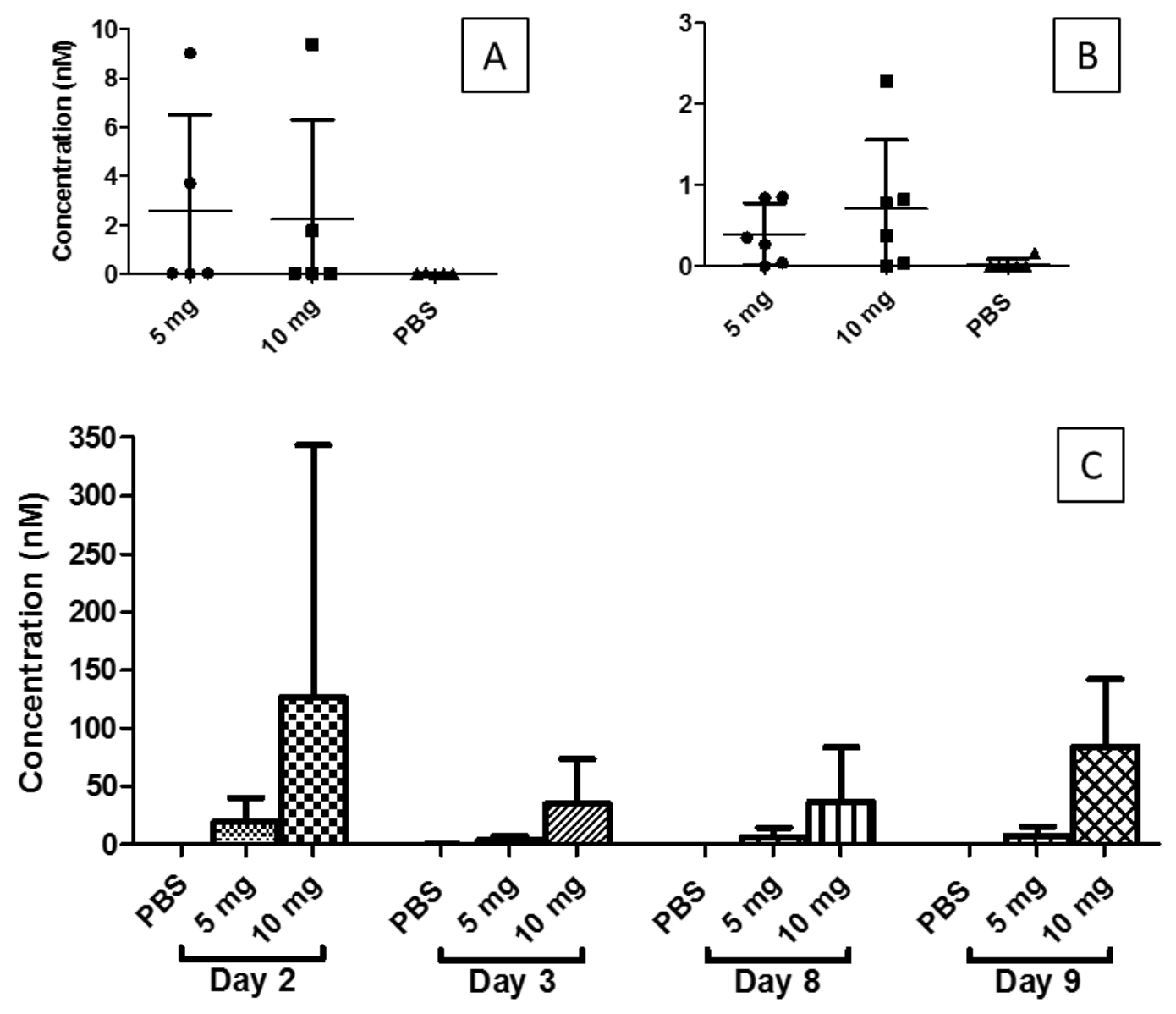

Figure 3.2. GRFT Concentrations in Fecal Extracts following Chronic Oral dosing. Mean GRFT concentrations from fecal extracts prepared from fresh fecal pellets obtained 8 hours (A) and 24 hours (B) after oral dosage with either PBS, 5 $\mathrm{mg}$ GRFT, or $10 \mathrm{mg}$ GRFT. Bars indicate mean \pm standard deviation of 5 biological replicates. Mean GRFT concentrations of fecal extracts prepared from desiccated pellets randomly drawn from cages (two animals per cage) on select days $(C)$. Bars indicate mean \pm standard deviation of 3 biological (cage) replicates. 


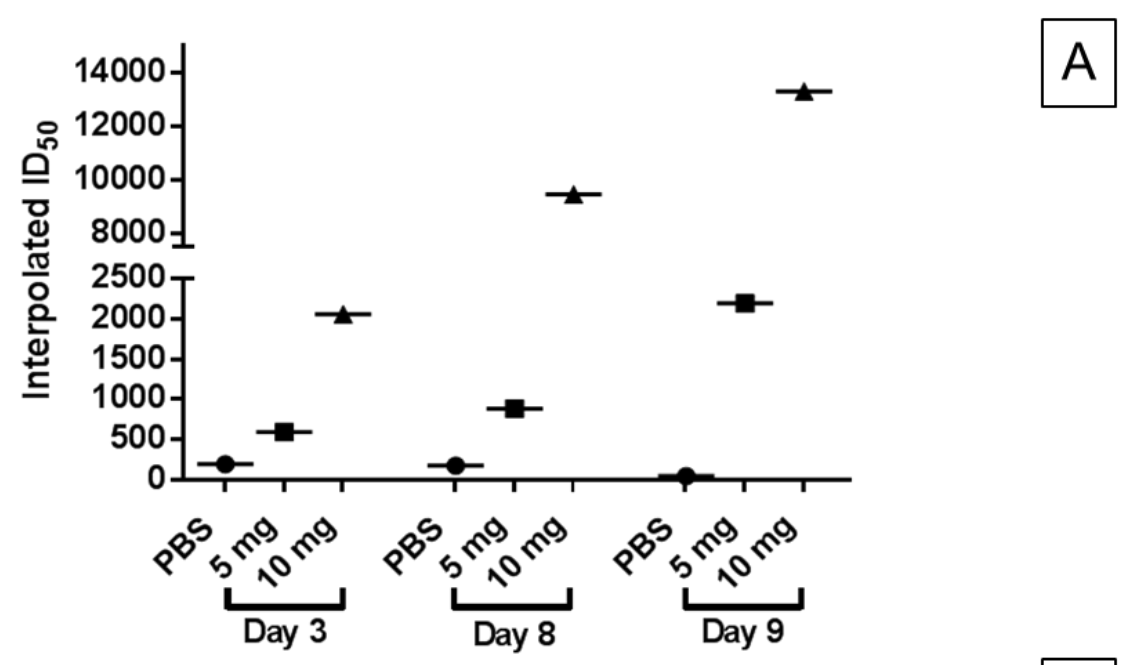

\begin{tabular}{|c|c|c|c|}
\hline Day & Treatment & $\begin{array}{c}\text { GRFT } \\
\text { Concentration } \\
\text { (nM) }\end{array}$ & $\begin{array}{c}\text { Interpolated } \\
\text { ID50 }\end{array}$ \\
\hline \multirow{3}{*}{3} & PBS & 0 & 192 \\
\hline & 5mg GRFT & 1.8 & 590 \\
\hline & 10mg GRFT & 35 & 2059 \\
\hline \multirow{3}{*}{8} & PBS & 0 & 174 \\
\hline & $5 \mathrm{mg}$ GRFT & 3.6 & 885 \\
\hline & 10mg GRFT & 36 & 9452 \\
\hline \multirow{3}{*}{9} & PBS & 0 & 43 \\
\hline & 5mg GRFT & 11 & 2195 \\
\hline & 10mg GRFT & 84 & 13307 \\
\hline
\end{tabular}

Figure 3.3. Antiviral activity of pooled fecal extracts collected on select days during the course of chronic oral treatment with GRFT. HIV-1 env- pseudovirus neutralization activity $\left(\mathrm{EC}_{50}\right.$ of $\left.\mathrm{GRFT}=\sim 40 \mathrm{pM}\right)$ was assessed for pooled rat fecal extracts from animals dosed with either PBS, $5 \mathrm{mg}$ or 10mg GRFT and expressed as $\mathrm{ID}_{50}$ (dilution factor required to reduce luminescence to $50 \%$ of PBS treated controls) (A). The mean GRFT concentrations were determined and compared to interpolated $\mathrm{ID}_{50}$ values $(\mathrm{B})$. 


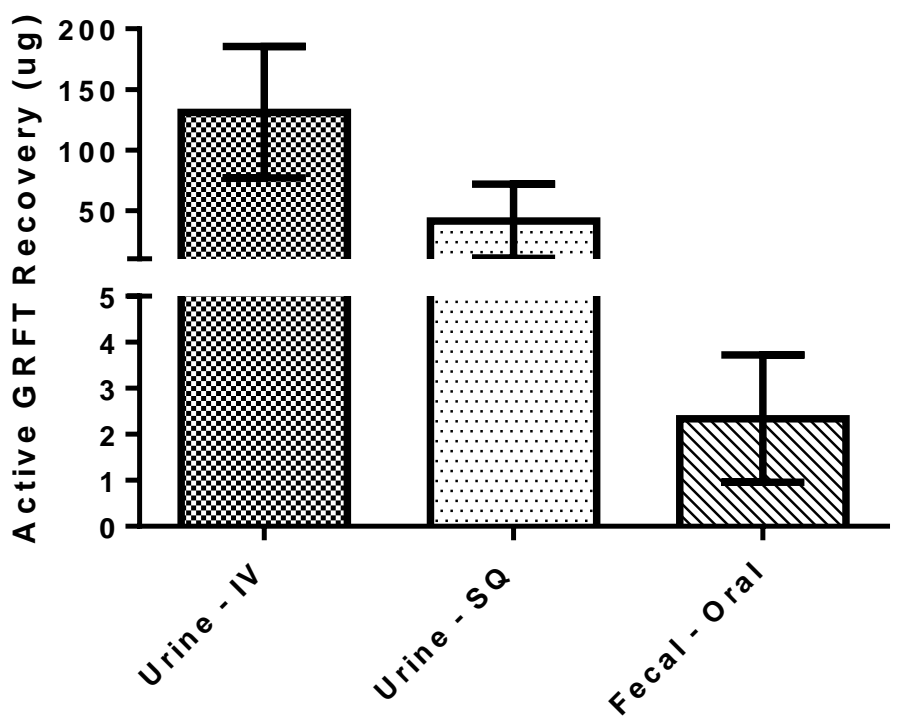

Figure 3.4. Total GRFT recovery following single administration. Based upon likely route of excretion, urine or fecal samples were obtained for animals receiving $10 \mathrm{mg} / \mathrm{kg}$ GRFT after Intravenous (IV), Subcutaneous (SQ), and oral administration. GRFT concentrations in urine and fecal extracts were determined and mean GRFT recovered was calculated. Bars indicate mean \pm standard deviation of 5 biological replicates. 


\title{
CHAPTER 4
}

\author{
SUB-CHRONIC SUBCUTANEOUS TREATMENT WITH THE ANTIVIRAL \\ LECTIN GRIFFITHSIN IN RATS TO DETERMINE GRIFFITHSIN'S SYSTEMIC \\ TOLERABILITY AND EXAMINE GRIFFITHSIN'S EFFECTS UPON ORGANS, \\ BLOOD CELL COUNTS, SERUM CHEMISTRIES, AND CYTOKINE \\ PRODUCTION

\section{INTRODUCTION}

The oligomannose glycan structures displayed on the surface of many human and animal pathogenic viruses are potential targets for antiviral therapeutics. CBAsact by targeting and binding to viral oligomannose glycans and include both lectin and non-peptidic drugs $[14,205]$. CBA relevance in viral therapy stems from the fact that, among enveloped viruses, glycans shields can be used for cellular entry, immune evasion, and in some cases, innate immune response suppression[13, 17, 19, 51, 60]. In vitro antiviral activity of CBAs against a broad array of enveloped viruses has been observed and verified by others $[28,29,189]$. However, most carbohydrate binding agents that display broad spectrum, strongly inhibitory antiviral activity are lectins that present 
several theoretical safety concerns for development as biologic therapies[14, 22, 24, 149]. Chief amongst the safety concerns are toxicities associated with offtarget activity, and immunotoxicities including hypersensitivity and production of anti-drug antibodies (ADA) that might neutralize the lectin antiviral activity and lead to undesirable side effects over long-term therapy.

One of the most promising antiviral lectins is the red alga Griffithsiaderived homodimeric lectin Griffithsin. GRFT (12.77 kDa) is a lectin that binds terminal mannose residues on $(\mathrm{N})$-linked $\mathrm{Man}_{5-9} \mathrm{GlcNAc}_{2}$ structures on viral envelopes $[32,163,165]$. These oligomannose glycans comprise the vast majority of N-linked glycans in the HIV-1 glycan shield [13, 16]. Consequently, GRFT has exceptional activity—in the mid picomolar range-against the heavily glycosylated lentiviruses HIV-1 and HIV-2[32, 33], and nanomolar inhibitory activity against other viruses of public health importance, such as HCV [30, 31], JEV [173] as well as SARS-CoV in addition to other CoV pathogens [23]. Data published by our group and others show that GRFT does not induce inflammatory cytokines on treatment of cultured human cells and primary lymphocytes; does not agglutinate human red blood cells; shows an excellent safety profile in highly sensitive preclinical safety models designed to predict toxicity at vaginal epithelia; and shows minimal toxicity after short-term oral, subcutaneous and intravenous exposure. Much like the in vivo studies into the efficacy of CBAs as antiviral treatments to inhibit influenza and Ebola viruses [28, 29], in vivo and ex vivo studies using GRFT in multiple murine virus challenge models have also shown promise. For example, intranasally administered GRFT 
prevents disease in mice challenged with SARS-CoV [172]. Intraperitoneal (IP) treatment with GRFT has prevented JEV [173]. Furthermore, GRFT showed efficacy against HCV in a mouse-human chimeric liver model when administered subcutaneously $[30,170]$.

Our previous studies have shown that an acute subcutaneous treatment of GRFT in guinea pigs and mice for a period of ten (10) days was tolerable with minimal toxicity reported [34]. We also showed that subcutaneous administration of GRFT at $10 \mathrm{mg} / \mathrm{kg}$ remains at potentially therapeutic concentrations up to 48 hours after administration, with therapeutically-relevant concentrations persisting for over a week after a single $50 \mathrm{mg} / \mathrm{kg}$ administration in mice [34]. However, as a xenogeneic protein, GRFT has the potential to elicit an immune response, up to and including anaphylaxis. Accordingly, longer term administration studies are necessary to assess GRFT's potential as a systemic therapeutic. Furthermore, the impact that ADA may have on GRFT's pharmacological activity, pharmacokinetics, and in vivo toxicity profile is unknown.

Here we report that minimal toxicity is induced by subcutaneous doses of GRFT in rats after approximately 8 weeks of treatment, though development of immune responses that may cause IgG ADA may require more prolonged and periodic monitoring. The drug remained systemically distributed, and accumulated at physiologically relevant levels after subcutaneous delivery for all time points tested. Inflammatory cytokine analysis at treatment cessation did not reveal widespread alterations in inflammatory cytokine levels, though some 
significant changes were observed in macrophage inflammatory proteins and leptin levels. Importantly we demonstrated that serum from GRFT treated animals retained anti-viral activity against HIV-1 envelope pseudoviruses in a cell line-based neutralization assay despite a modest ADA response to the GRFT. Overall, these findings support further investigation into GRFT's potential as a systemic antiviral therapeutic agent against enveloped viruses including HIV-1. 


\section{MATERIALS AND METHODS}

Lectin reagents

Recombinant GRFT was produced as described previously, purified to $>99 \%$ purity, and formulated in phosphate buffered saline (PBS), pH 7.4 [169].

Animal housing and care

225-250g Sprague Dawley Rats (Rattus Norvegicus, Charles River Laboratories), housed in a temperature- and humidity-controlled room with an alternating light/dark cycle of $12 \mathrm{~h}$, were provided a standard diet and water ad libitum. All experimental procedures utilized were approved by the University of Louisville's Institutional Animal Care and Use Committee.

Rat IgG Generation

To generate IgG antibodies for passive immunization, 9 Sprague Dawley rats $(250 \mathrm{~g}+)$ were injected subcutaneously with $500 \mu$ l Sigma Adjuvant (monophosphoryl lipid A, synthetic trehalose dicorynomycolate in $2 \%$ squaleneTween® 80-water) and GRFT formulated at $1 \mathrm{mg} / \mathrm{ml}$ per manufacturer's recommended protocol. Booster immunizations were performed according to manufacturer protocol every 3 weeks for 12 weeks. Two weeks after final immunization animals were sacrificed by $\mathrm{CO}_{2}$ asphyxiation and serum was collected via cardiac puncture. Ig from these samples was purified by NAB spin column (Sigma) according to the manufacturer's protocol. For nonspecific 
controls, IgG from commercial Sprague Dawley rat serum (InnovoResearch) was purified by NAB spin column (Sigma). Ig was then dialyzed (Thermo 3500 mwco) against PBS to remove elution buffer and sterile filtered prior to subcutaneous administration.

Rat treatment and sample collection

Age-matched, $225 \mathrm{~g}$ Sprague Dawley rats were allowed to a week to acclimate before treatment according to the treatment regimen diagram shown in Figure 4.1. After acclimation, two groups were pre-immunized with either $0.5 \mathrm{ml}$ of Sigma Adjuvant and GRFT at $1 \mathrm{mg} / \mathrm{ml}(\mathrm{n}=7)$ or $0.5 \mathrm{ml}$ Sigma Adjuvant and PBS $(n=6)$ before commencing treatment. Pre-immunized animals were boosted twice at three week intervals. Animals not undergoing pre-immunization were left fallow during the pre-immunization phase. Treatment groups consisted of the following: pre-immunized with Adjuvant and PBS $(n=6)$, pre-immunized with GRFT and Adjuvant ( $n=7)$, GRFT treated $(n=7)$, PBS treated $(n=6)$, GRFT treated and passively immunized with anti-GRFT Ig $(n=7)$, PBS treated and passively immunized with nonspecific $\lg (n=6)$. Animals were treated subcutaneously with either PBS or $10 \mathrm{mg} / \mathrm{kg}$ GRFT every three days. Passively-immunized animals also received $1.4 \mathrm{mg}$ of appropriate $\mathrm{lg}$ formulated in $1 \mathrm{ml}$ of PBS every 14 days.

Serum was drawn via lateral tail vein on the following days: Day 10, 24, 38 , and 52 . Animals were sacrificed on day 55 via $\mathrm{CO}_{2}$ asphyxiation followed by cardiac puncture exsanguination. Blood, hearts, lungs, kidneys, liver, spleen, colon, and vaginas were collected at sacrifice for analysis. 
GRFT capture immunoassay using the HA glycoprotein.

To detect active concentrations of GRFT present in serum we used an Influenza HA binding ELISA. Briefly, Maxisorp plates (Nunc) were coated with 10 $\mu g$ purified HA (Kentucky Bioprocessing) and incubated overnight at $4^{\circ} \mathrm{C}$. Plates were blocked with $\sim 3 \%(\mathrm{w} / \mathrm{v})$ Bovine Serum Albumin (BSA) in PBS-T. Serum samples were diluted 1:10 in blocking buffer. Serial dilutions of purified GRFT were run in parallel for generation of a standard curve. Serum and GRFT dilutions and were incubated at RT for 1 hour. HA-bound GRFT was detected by rabbit anti-GRFT antiserum (1:25,000) followed by HRP-conjugated goat antirabbit IgG $(1: 10,000)$. Plates were developed with SureBlue TMB Microwell Peroxidase Substrate, with reactions stopped with $1 \mathrm{~N} \mathrm{H}_{2} \mathrm{SO}_{4}$ after 10 minutes of development. Absorbance readings at $450 \mathrm{~nm}$ and $570 \mathrm{~nm}$ wavelengths were measured using a BioTek Synergy HT plate reader. Concentrations were determined by Nanoanalyze software.

\section{Evaluation of Anti-HIV Activity.}

HIV-1 neutralization activity of heat-inactivated serum samples obtained on day 52 and 55 was measured using pseudovirus neutralization assays as previously described [174]. Briefly, DU156 env-pseudotyped virus particles generated by transfection of 293T cells were titrated in TZM-bl cells both in the presence and absence of serum sample dilutions. Antiviral activity of the serum samples was calculated as a function of luciferase activity. $I D_{50}$ values were 
defined as the sample dilution required to reduce well luminescence by $50 \%$ in comparison to wells with no sample added.

Hematology parameters and Serum Chemistry.

A complete blood count ( $\mathrm{CBC}$ ) was run for terminal bleed samples using a Hemavet $₫ 950$ (Drew Scientific) standardized for rat blood. Parameters quantified in potassium-EDTA anti-coagulated whole blood included: red blood

cells $\left(\mathrm{RBC} ; 10^{4} / \mu \mathrm{l}\right)$, total and differential leukocyte count (neutrophils, lymphocytes, monocytes, eosinophils, and basophils as $10^{3} / \mu$ or $\%$ ), hemoglobin concentration (HGB; g/dl), hematocrit (HCT; \%), mean corpuscular volume (MCV; $\mathrm{fl})$, mean cell hemoglobin $(\mathrm{MCH} ; \mathrm{pg})$, mean cell hemoglobin concentration (MCHC; g/dl), red cell distribution width (RDW; \%), platelets (PLT; 10 $/ \mu \mathrm{l})$, and mean platelet volume (MPV; fl).

Serum chemistry panels were also run for terminal bleed samples using a VetTest ${ }^{\circledR}$ Chemistry Analyzer (IDEXX Laboratories). Parameters quantified from serum included: serum albumin (Alb), alkaline phosphatase (ALKP), amylase (Amy), alanine aminotransferase (ALT), blood urea nitrogen (BUN), calcium (Ca), cholesterol (Chol), creatinine (Creat), globulin (Glob), glucose (Glu), phosphorus (Phos), total bilirubin (TBil), and total protein (TP).

Rat Antibody titration

To quantitate degree of immune response to GRFT, antibody titers were determined for terminal bleed samples (day 55) using a sandwich ELISA. Briefly, 
Maxisorp plates (Nunc) were coated with 5 ug purified HA (Kentucky

Bioprocessing) and incubated overnight at 4 C. Plates were blocked with $\sim 3 \%$ (w/v) Bovine Serum Albumin (BSA) in PBS-T. 10ug/ml GRFT (to excess) was diluted in blocking buffer and incubated at RT for 1 hour. Serial dilutions of sample sera diluted in PBS-T containing 2M urea were incubated in wells for 2 hours. GRFT-bound rat Ig was detected by HRP-conjugated Donkey anti-rat polyclonal antibody (1:25,000). Plates were developed with SureBlue TMB Microwell Peroxidase Substrate, with reactions stopped with 1N H2SO4 after 10 minutes of development. Absorbance readings at $450 \mathrm{~nm}$ and $570 \mathrm{~nm}$ wavelengths were measured using a BioTek Synergy HT plate reader. Final absorbance 2 times background was considered a positive signal for a particular dilution.

\section{Cytokine Quantitation}

To measure any inflammatory effects of chronic exposure, cytokine levels in samples drawn on day 52 were determined by Milliplex Map Kit (Millipore) using manufacturer's standard protocol. The following cytokine levels were quantified: granulocyte colony-stimulating factor (G-CSF), Eotaxin, Granulocyte macrophage colony-stimulating factor (GM-SCF), Interleukin 1 alpha (IL-1a), Leptin, Macrophage Inflammatory Protein 1 alpha (MIP 1-a), Interleukin 4 (IL4), Interleukin 1 beta (IL-1B), Interleukin two (IL-2), EGF, Interleukin 13 (IL-13), Interleukin 10 (IL-10), Interluekin 12 (IL-12p70), Interferon gamma (INFy), Interleukin 5 (IL-5), Interleukin 17 alpha (IL-17A), Interleukin 18 (IL-18), 
monocyte chemoattractant protein 1 (MCP-1), interferon gamma induced protein 10 (IP-10), growth-related oncogene (GRO/KC), vascular endothelial growth factor (VEGF), Fractalkine, lipopolysaccharide induced CXC chemokine (LIX), macrophage inflammatory protein 2 (MIP-2), tumor necrosis factor alpha (TNFa), and Rantes.

Statistical Analysis.

Statistical analysis was conducted using Graph Pad Prism 5. Two-way ANOVA was utilized. A p-value $<0.05$ was deemed significant. Bonferonni posttests were conducted to determine whether any differences were attributable to GRFT treatment. 


\section{RESULTS}

GRFT serum concentrations.

Serum samples collected from all rats treated with $10 \mathrm{mg} / \mathrm{kg}$ GRFT showed nanomolar concentrations of GRFT at every time point tested (Figure 4.2A).

These levels predictably varied by time since last dosage, which ranged from 24 to 72 hours after administration. In Day 52 sera (24 hours after final administration), all groups treated with GRFT displayed active mean GRFT concentrations of approximately $10 \mathrm{nM}$. Notably, these concentrations of GRFT persisted at mean levels of approximately $4 \mathrm{nM}$, regardless of GRFT treatment subgroup, at 96 hours after dosing. Serum concentrations from GRFT treated animals were also compared to serum concentrations from prior single subcutaneous administration studies described in Chapter 3 (Figure 4.2B). Mean GRFT concentrations in animals chronically treated with GRFT were higher at all time points versus serum concentrations from animals receiving a single dose.

Anti-GRFT and Anti-HIV Activity of serum.

Serum Samples displayed varying levels of Anti-GRFT activity based upon treatment group (Figure 4.3A). GRFT treated animals (without pre-immunization) showed GRFT titers slightly elevated over background. Animals receiving passive Ig immunization and pre-immunization with GRFT and adjuvant had the highest mean activity titrations at 1:767 and 1:1542 respectively. There was clear 
variability in the GRFT antibody titers in both the pre-immunized and passively immunized groups.

Serum samples collected from rats treated with $10 \mathrm{mg} / \mathrm{kg}$ GRFT neutralized HIV-1 pseudoviruses (Clade C primary sexually transmitted isolate Du156) with an overall mean $I D_{50}$ of approximately 20500 on experimental day 52 (Figure 4.3B). The antiviral activity measured in rat sera was not statistically different between animals that were immunologically naïve at the study outset (GRFT group) versus the preimmune group and the passively immunized group. After a 96 hour recovery period, group mean $I D_{50}$ values decreased to approximately 4300 on day 55 . PBS treated rat sera displayed a mean $I D_{50} S$ of 264 and 112 on days 52 and 55, respectively. Both pre-immunized and passively immunized groups also receiving GRFT treatment displayed strong neutralization activity on Day 52 as well, with mean ID50 values of 19800 and 17000 respectively. These group mean $I D_{50} s$ also decreased 96 hours after treatment, similarly to animals treated only with GRFT. Rat neutralization titers did not correlate well with GRFT's published anti-HIV-1 EC50 of $40 \mathrm{pM}$, likely as a product of background neutralization activity observed in rat serum.

GRFT is well tolerated after chronic subcutaneous administration

We studied the toxicity of GRFT in rats using several parameters including mortality, behavior, animal body and organ weight changes, tissue pathology, and changes in blood properties. 
All animals survived treatment with no behavioral changes observed. Body weight, as a surrogate marker for overall fitness following GRFT treatment, was evaluated. Animals were weighed at day 1 and day 55. Using a 2-way ANOVA, we found no significant changes in starting weight or weight gain among treatment groups $(p=0.4177$ and $p=0.9170$ respectively, Figure 4.4$)$.

Liver, kidney, and spleen weights were measured at sacrifice and normalized to total body weights. No significant changes of the normalized weights of livers were observed $(p=0.1487$, Figure 4.4C) However, animals passively immunized with GRFT IgG and treated with GRFT displayed significantly lower kidney/body weight ratios in comparison to PBS treated controls $(p=0.0328$, Figure 4.4D). Additionally, we observed a trend toward larger spleen weights in all animals treated with GRFT, regardless of whether they were pre-immunized $(p=0.0561$, Figure 4.4E).

Tissue sections were stained with hematoxylin and eosin and evaluated in a blinded fashion by a veterinary pathologist (MaryProctor). The pathologist noted differences among individual animals that were attributable to sample preparation and sectioning. However, no treatment-related pathologies were observed for any treatment group.

CBC results displayed no significant changes among groups though Red Blood Cell Width and Platelet values approached significance with $p$-values of 0.0977 and 0.0796 respectively (Table 4.1 ). Serum chemistries displayed some statistically significant differences among treatment groups (Table 4.2). Among 
these were albumin, amylase, phosphorus, blood urea nitrogen, and globulin. Bonferroni post-test identified differences among treatment groups. Phosphorus levels were significantly higher in vehicle-only (PBS) than in all GRFT treated groups $(p=0.0083)$. Amylase and globulin levels were significantly higher in Passively Immunized/GRFT treated animals though amylase levels were within the normal range for rats $(p=0.0468$ and $p=0.002$ respectively). Finally, albumin levels were significantly lower in pre-immunized/GRFT treated animals than in PBS treated controls, though within the normal range for rats $(p=0.0071)$.

\section{Cytokine responses}

Cytokine panels with a minimum of 3 positive signals per group were analyzed. Analysis of PBS, GRFT, Pre-Imm/GRFT, and Pass-Imm/GRFT revealed only 4 significant differences among the cytokines examined: Leptin, MIP-1a, Ip-10, and MIP-2 (Table 3). In the case of leptin, all groups treated with GRFT had significantly lower leptin levels than PBS treated controls $(p=<0.0001)$. Further, IP-10 levels were lower in the group receiving GRFT-only treatment $(p=0.0312)$. MIP-1a elevation was seen in the pre-immunized/GRFT treated group $(p=0.018)$. However, posttest analysis did not reveal significance versus PBS-treated controls, but rather significance versus GRFT-only treated animals. Finally, MIP-2 displayed a significant difference among treatment groups $(p=0.0382)$. However, post-test analysis failed to identify differences between individual groups. 


\section{DISCUSSION}

In this study, we demonstrate that intermediate term sub-chronic GRFT administration is tolerated in rats, despite high levels of experimentally induced ADA. Levels of ADA varied among groups and also varied widely within groups. High anti-GRFT antibody titers were not observed in any animal even when preimmunized with adjuvant. Regardless of ADA levels, GRFT persisted in serum at concentrations above the $\mathrm{EC}_{50}$ described for multiple enveloped viruses $[23,31$, $32,178]$. Further these levels were observed in all GRFT treated animals, at all time points tested, and up to 96 hours after administration. Although predictably decreasing after final administration, functionally active GRFT concentrations--as determined by HA binding ELISA and HIV-env pseudovirus neutralization assay-remain in circulation for many days after treatment cessation. These findings support the continued study of GRFT as a viral therapeutic.

As noted in prior studies, a favorable safety profile for a drug must be demonstrated to advocate for its further development [34, 35]. We observed previously that GRFT was capable of persisting in the system for many days after administration at potentially therapeutic levels when subcutaneously or intravenously dosed ([34] and manuscript in preparation). Thus greater time between administrations was selected to mimic a more favorable dosing regimen that would be more acceptable for those relying upon subcutaneous selfadministration while not allowing GRFT to completely clear systemic circulation. Based upon prior work, which described GRFT's clearance in rats following 
subcutaneous administration at $10 \mathrm{mg} / \mathrm{kg}$ at $0.058 \mathrm{~L} / \mathrm{hr}$, a dosing regimen of 2.5 mg every 72 hours was calculated to generate steady state concentrations of GRFT of 47 nM, over 1000 times GRFT's anti HIV-1 EC50 of 40 pM. Further, this extended dosing regimen was sufficient to keep GRFT in the system at concentrations well above its $\mathrm{EC}_{50}$ for HIV at 96 hours after final dose, thus suggesting longer potential periods between administrations in humans may also be possible, if not ideal. Injection administration of antiviral therapies is not unheard of, and research is currently being conducted into long acting anti-viral medications for HIV prevention and treatment [206-208]. Accordingly, efforts to prolong GRFT's serum persistence may be necessary to further enhance an already acceptable administration profile.

In these current in vivo studies in rats, GRFT was not observed to alter experimental animal behavior, nor did any animal die as a result of treatment. Potential organ toxicity was also assessed by visual inspection during necropsy, weight measurements and histopathology. No differences in organ appearances were observed during necropsy. While kidney weight percentages of total body weight in passively immunized/GRFT treated animals displayed a statistically significant lower percentage in comparison with PBS-treated controls and preimmunized/GRFT treated animals trending toward lower percentages as well, histopathological examination of the organs did not show any treatment-related pathologies. Given the animals in this study were still growing, these lower percentages may be a result of either impaired kidney growth or potential nephrotoxicity. A prior study (manuscript currently in preparation) showed a 
significant excretion of active GRFT into urine after both intravenous and subcutaneous systemic administration. Accordingly, given that the observed changes in kidney weights were seen in animals in which had significantly higher globulin levels, it is possible that GRFT-antibody complexes or some component on an immune response have an impact upon the renal function. Further supporting this possibility was the observed trend toward lower kidney weight and higher globulin levels also observed in pre-immunized/GRFT treated animals. Accordingly, further study will be necessary to determine how an antibody response impacts kidney function given that no histopathological abnormalities were observed by the veterinary pathologist (M.P.).

When a complete blood count was performed and serum chemistries analyzed, we observed that no CBC parameters were significantly changed as a result of GRFT treatment. However, serum chemistries did show some variation. Post hoc analysis revealed that amylase and globulin levels were significantly higher in passively-immunized/GRFT treated animals versus PBS treated controls. While amylase levels were still within the normal range for rats, elevated globulin levels could reasonably be expected as an outcome of Ig administration, and thus could be a confounding effect derived from the experimental treatment.

Significant differences in albumin and BUN in pre-immunized animals versus PBS treated controls were observed. Interestingly, the difference in BUN combined with the trend toward a lower kidney to bodyweight ratio may suggest a degree of kidney impairment associated with an immune response to GRFT 
which impairs kidney excretion of urea, as elevated BUN levels can be indicative of kidney malfunction $[209,210]$. Finally, a difference in phosphate levels was observed in PBS treated animals in comparison with all GRFT treated groups. However, closer analysis revealed that the PBS group average may have been skewed by two high values and all values for phosphate were within the physiological normal range for rats.

While prior studies have demonstrated that GRFT is not mitogenic, is not cytotoxic to cells, and does not induce cell mediators of inflammation [35], a major concern with an immune response to GRFT is that, as a xenogenic protein, anaphylaxis is possible [180]. Although GRFT is a relatively weak immunogen, we were able to raise a modest IgG antibody response in animals pre-immunized with GRFT in the presence of adjuvant. Fortunately, it appears based upon both ELISA and pseudovirus neutralization assay that immune sera are binding, but non-neutralizing.

Immunogenicity of biologic drugs such as GRFT is a known issue and in this study we demonstrate that while tolerable, some physiological changes may be induced by an $\lg G$ based immune reaction to GRFT. Despite differing $\lg G$ models of an immune response, it appears that GRFT administration does not significantly impact the majority of cytokines examined. This corroborates prior studies suggesting that GRFT does not induce cell mediators of inflammation [35]. Nonetheless, there were some notable exceptions. The most significant difference observed was in leptin levels. Leptin is a hormone made by body fat 
to regulate food intake and body weight. However, it has also been implicated in T-cell regulation as well as inflammation, wherein leptin is considered proinflammatory and proangiogenic [211]. These findings of decreased leptin levels in all GRFT treated groups may indicate some anti-inflammatory effect of GRFT treatment. However, further studies into GRFT's interactions with leptin are needed to determine the exact mechanism by which leptin levels are lowered, though interestingly, a lower body weight gain was observed in guinea pigs treated with GRFT in a previous study [34]. This same conclusion may be drawn from IP-10 levels. IP-10 (interferon inducible protein-10) levels were significantly lower in animals receiving GRFT treatment in absence of pre-immunization or simulated immune response. Thus, in animals in which no immune response was generated or simulated, IP-10 levels were seen to decrease, further supporting the possibility that GRFT may have some discrete anti-inflammatory activity. However, once an immune response was initiated, it seems this effect may disappear. MIP-1a and MIP-2 were elevated in animals pre-immunized with GRFT and adjuvant. In the case of MIP-1a, the elevation was significant in preimmunized/GRFT treated animals versus PBS treated controls. In the case of MIP-2, ANOVA post hoc analysis did not reveal the source of the variation. However, both GRFT and GRFT/passively immunized animals displayed decreased levels of MIP-2 verses PBS controls, while the pre-immunized GRFT treated group was elevated versus PBS controls. While further study into the exact effect of GRFT on these cytokines will be necessary, these results seem to suggest that GRFT may function to slightly decrease inflammation. These results 
also raise the possibility that GRFT may have an immunosuppressive effect. Further immune suppression would likely be undesirable in HIV disease and may present a barrier to usage in those with existing infections. Additionally, the specter of immunosuppression may require additional testing, such as viral, bacterial, and tumor infection challenges as well as a full complement of in vitro cell activation assays to ensure that GRFT does not prevent humoral or adaptive immune response to other pathogens.

Resistance to CBAs occurring through a loss of specific $\mathrm{N}$-linked glycosylation sites has been observed when those viruses are placed under drug pressure with CBAs $[25,185-188]$. In HIV-1, CBA resistance has been correlated with reduced viral fitness leading to a potentially enhanced susceptibility to endogenous antibody neutralization $[14,186,188]$. Thus it has been hypothesized by Balzarini and others that CBA therapy may induce resistance to the CBA while enhancing susceptibility to immune system neutralization, particularly in the case of HIV-1[14]. GRFT's continued antiviral activity even in presence of an immune response, as well its $\sim 8$ week tolerability profile, would seem to indicate that GRFT is a candidate drug to test that hypothesis, as it could be used for longer intermittent periods in attempts to guide viral resistance evolution.

These results demonstrate that despite both pre-immunization and passive immunization, no animal mortality was observed in any treated animal. Furthermore, unlike our prior studies in guinea pigs and mice, our current data 
does not show significant treatment-associated increases in spleen and liver mass-to-bodyweight ratios, though spleen differences approached significance, probably associated with an immune response to GRFT. However, it does appear that an immune response may result in kidney involvement, the exact nature of which is not clear at present. As such, future efforts to de-immunize GRFT may be necessary $[161,162]$, before it is viable as a long-term chronic treatment of viral infection in humans. Nonetheless, we conclude that the preliminary toxicity profile of GRFT is acceptable and encourages its further development as an intermediate term antiviral prophylaxis and therapy. 


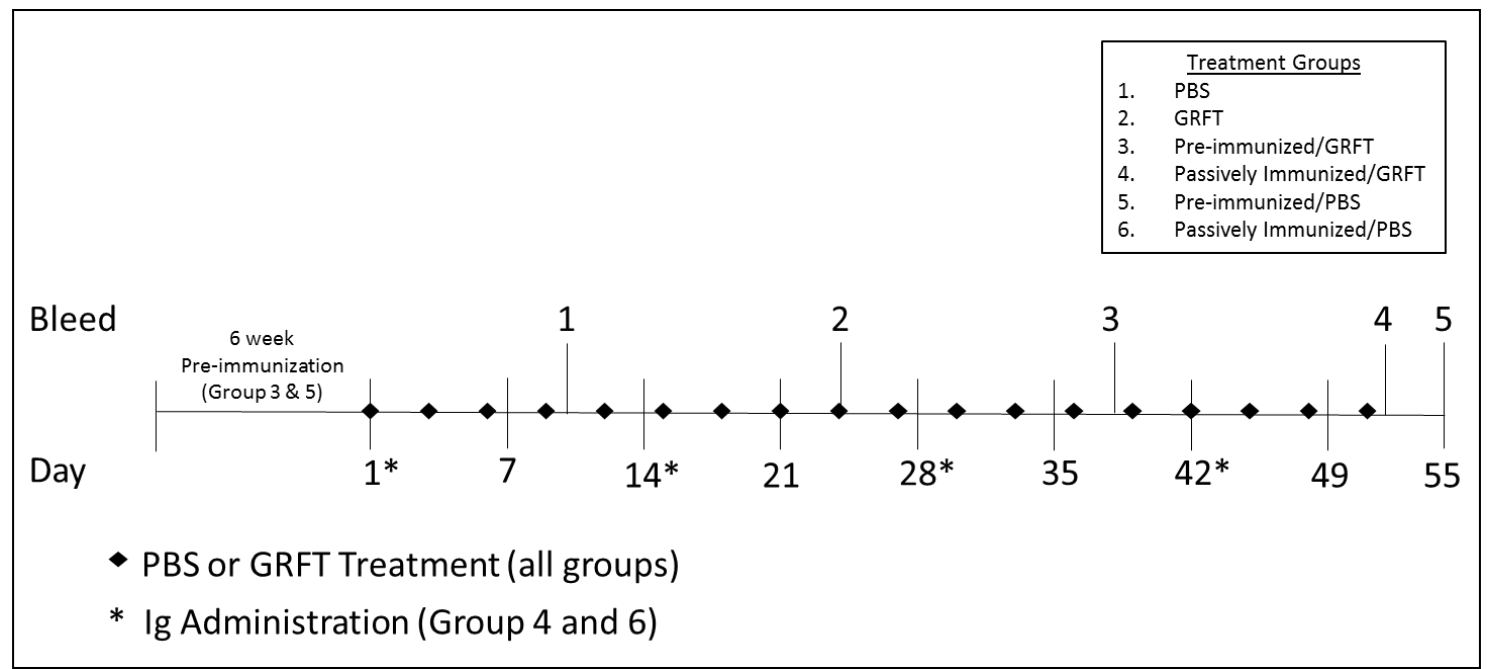

Figure 4.1. Treatment Regimen to evaluate the impact of an immune response upon GRFT's systemic tolerance and activity. Animals were separated into groups of 6-7 animals and dosed every 3 days with either PBS or GRFT during course of treatment. Select groups were pre-immunized prior to treatment commencement (3 and 5) or passively immunized with IgG during chronic treatment (4 and 6). 


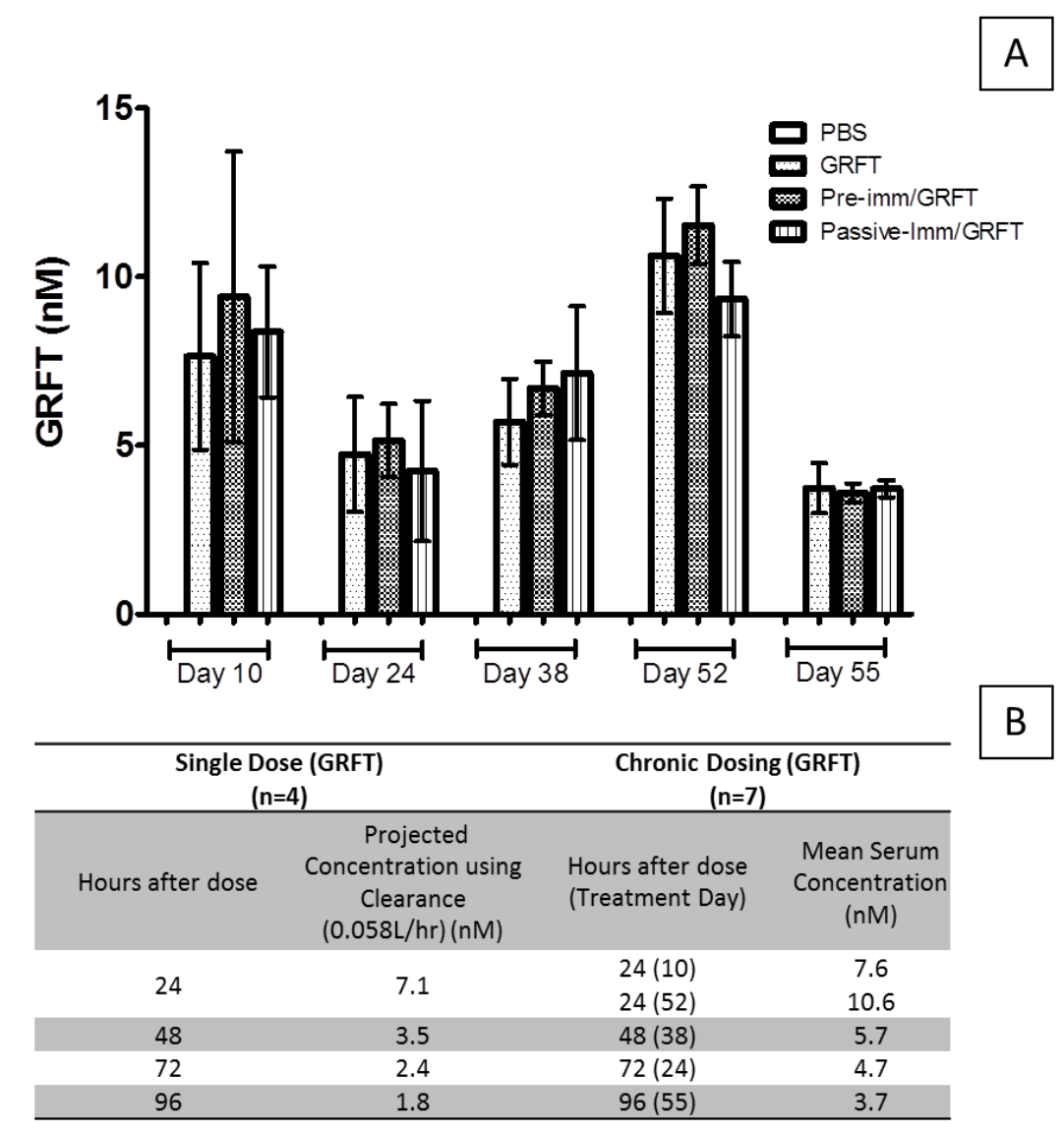

Figure 4.2. GRFT serum concentrations in rats during and after chronic GRFT treatment and comparisons with single dose pharmacokinetic values. (A) GRFT concentrations were determined (12.77 kDA) from rat sera of naïve, preimmunized (pre-imm), or passively immunized (passive-imm) animals following chronic PBS or 10mg/kg GRFT administration on day 10 (24 hrs after dosage), day 24 (72 hours after dosage), day 38 (48 hours after dosage), day 52 (24 hours after dosage), and day 55 (96 hours after dosage). PBS was administered to control animals Error bars indicate standard deviation of 7 biological replicates. (B) Mean GRFT concentrations of chronic dosing and a single subcutaneous dose (projected using Clearance value of $0.058 \mathrm{~L} / \mathrm{hr}$ ) reflect minor GRFT accumulation during treatment. 


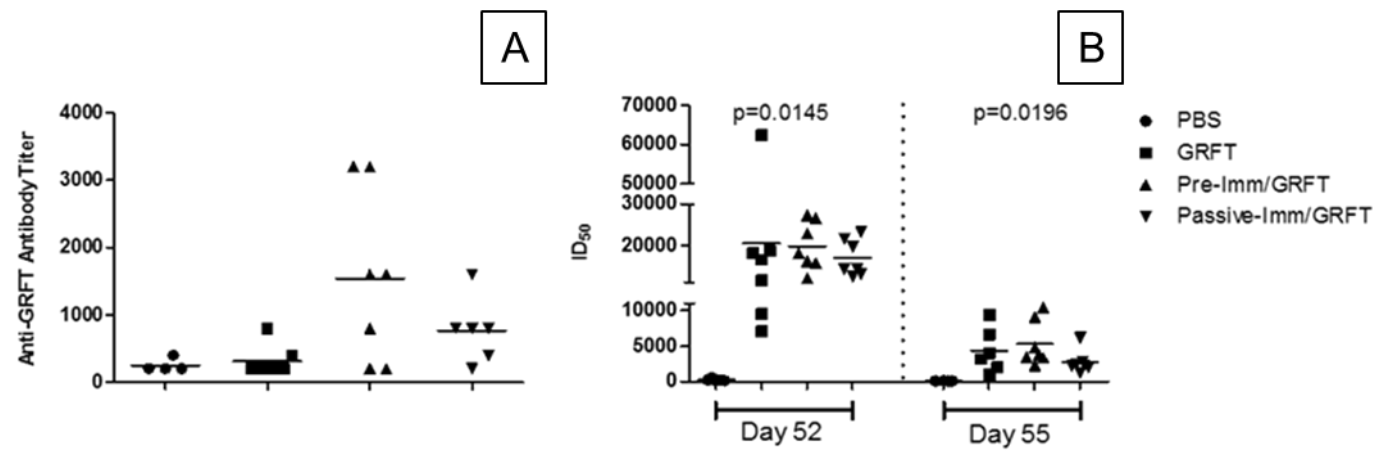

Figure 4.3. Titers of anti-GRFT IgG and antiviral activity of sera from rats preimmunized against GRFT, passively immunized with GRFT IgG, and controls. Anti-GRFT activity of Rat serum at Day 55 was evaluated via titration (A). HIV-1 env-pseudovirus neutralization activity was assessed for rat sera 24 hours after final administration of GRFT (Day 52) and 96 hours after final administration (Day 55) (B). Antiviral activity was expressed as $I D_{50}$. Control animals were treated with PBS. Anti-HIV-1 EC50 of GRFT $=\sim 40$ pM. Bars indicate mean group concentration. 

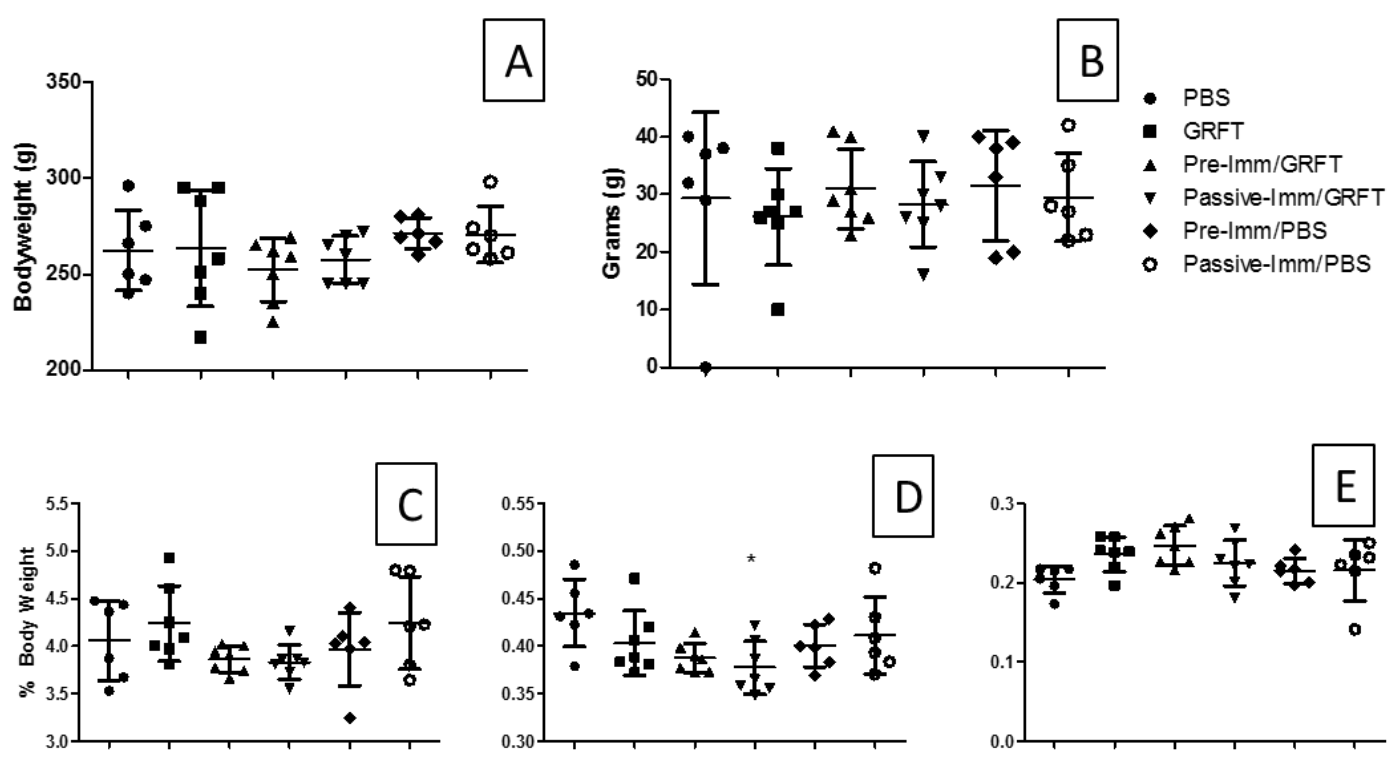

Figure 4.4. Starting weight, weight gain over treatment, and Organ percentages from rats preimmunized against GRFT, passively immunized with GRFT IgG, and controls. Animal bodyweights were measured on experimental day $1(A)$ and at termination. Weight gain over treatment was calculated for each animal (B). Liver (C), Kidney (D) and Spleen (E) weights were measured relative to body weight at sacrifice. Bars indicate group mean and standard deviation of 6 to 7 biological replicates. Significant $(p<0.05$, One way ANOVA with Bonferroni posttest) is indicated by $\left(^{*}\right)$. 


\begin{tabular}{c|ccccccc}
\multicolumn{1}{c}{} & \multicolumn{1}{c}{$\begin{array}{c}\text { PBS } \\
\text { Cell Type }\end{array}$} & Parameter & Units & $\begin{array}{c}\text { GRFT } \\
(n=6)\end{array}$ & $\begin{array}{c}\text { Pre-Imm/ } \\
\text { GRFT } \\
(n=7)\end{array}$ & $\begin{array}{c}\text { Pass-Imm/ } \\
\text { GRFT } \\
(n=6)\end{array}$ & $\begin{array}{c}P \text { - } \\
\text { value }\end{array}$ \\
\hline Leukocyte & $\mathrm{WBC}$ & $\mathrm{K} / \mu \mathrm{L}$ & $7.86 \pm 2.22$ & $8.98 \pm 2.80$ & $10.53 \pm 3.90$ & $8.75 \pm 4.82$ & 0.5978 \\
& $\mathrm{Ne}$ & $\mathrm{K} / \mu \mathrm{L}$ & $2.63 \pm 0.60$ & $3.05 \pm 1.16$ & $3.66 \pm 1.30$ & $2.77 \pm 1.50$ & 0.4281 \\
& $\mathrm{Ly}$ & $\mathrm{K} / \mu \mathrm{L}$ & $4.62 \pm 1.59$ & $5.35 \pm 1.60$ & $6.04 \pm 2.45$ & $5.37 \pm 3.02$ & 0.7317 \\
& $\mathrm{Mo}$ & $\mathrm{K} / \mu \mathrm{L}$ & $0.37 \pm 0.21$ & $0.41 \pm 0.24$ & $0.62 \pm 0.32$ & $0.38 \pm 0.33$ & 0.3267 \\
& $\mathrm{Eo}$ & $\mathrm{K} / \mu \mathrm{L}$ & $0.19 \pm 0.10$ & $0.14 \pm 0.13$ & $0.16 \pm 0.18$ & $0.16 \pm 0.10$ & 0.9540 \\
& $\mathrm{Ba}$ & $\mathrm{K} / \mu \mathrm{L}$ & $0.05 \pm 0.04$ & $0.03 \pm 0.05$ & $0.04 \pm 0.04$ & $0.05 \pm 0.02$ & 0.9269 \\
& $\mathrm{RBC}$ & $\mathrm{M} / \mu \mathrm{L}$ & $6.63 \pm 0.51$ & $6.33 \pm 0.79$ & $6.55 \pm 0.54$ & $6.07 \pm 1.39$ & 0.6663 \\
& $\mathrm{Hb}$ & $\mathrm{g} / \mathrm{dL}$ & $14.0 \pm 0.6$ & $13.7 \pm 0.7$ & $13.4 \pm 1.3$ & $12.5 \pm 2.3$ & 0.2428 \\
& $\mathrm{HCT}$ & $\%$ & $37.0 \pm 1.9$ & $34.2 \pm 4.8$ & $35.2 \pm 2.8$ & $32.7 \pm 8.0$ & 0.4891 \\
& $\mathrm{MCV}$ & $\mathrm{fL}$ & $55.9 \pm 2.8$ & $54.0 \pm 2.8$ & $53.7 \pm 1.1$ & $53.7 \pm 1.4$ & 0.2442 \\
& $\mathrm{MCH}$ & $\mathrm{pg}$ & $21.2 \pm 1.0$ & $21.9 \pm 3.4$ & $20.4 \pm 0.9$ & $20.8 \pm 1.6$ & 0.5537 \\
& $\mathrm{MCHC}$ & $\mathrm{g} / \mathrm{dL}$ & $37.9 \pm 1.4$ & $40.8 \pm 7.4$ & $38.0 \pm 1.6$ & $38.7 \pm 4.1$ & 0.6119 \\
& $\mathrm{RDW}$ & $\%$ & $14.0 \pm 0.5$ & $13.8 \pm 0.6$ & $14.4 \pm 0.4$ & $14.4 \pm 0.4$ & 0.0977 \\
& $\mathrm{Plt}$ & $\mathrm{K} / \mu \mathrm{L}$ & $859 \pm 314$ & $890 \pm 325$ & $655 \pm 185$ & $542 \pm 184$ & 0.0796 \\
& $\mathrm{MPV}$ & $\mathrm{fL}$ & $7.5 \pm 0.4$ & $7.6 \pm 0.3$ & $7.5 \pm 0.4$ & $7.7 \pm 0.4$ & 0.7263
\end{tabular}

Table 4.1. Hematological profile for rats after chronic treatment with GRFT.

Data represent the mean values +/- standard deviation for white blood cells (WBC), neutrophils (NE), lymphocytes (LY), monocytes (MO), eosinophils (EO), basophils (BA), red blood cells (RBC), hemoglobin $(\mathrm{Hb})$, hematocrit $(\mathrm{HCT})$, mean corpuscular volume (MCV), mean cell hemoglobin $(\mathrm{MCH})$, mean cell hemoglobin concentration (MCHC), red cell distribution width (RDW), platelets $(P L T)$, and mean platelet volume (MPV). Significance $(p<0.05, A N O V A)$ is indicated by $\left({ }^{*}\right)$. 


\begin{tabular}{|c|c|c|c|c|c|c|c|}
\hline & UNITS & $\begin{array}{l}\text { NORMAL } \\
\text { RANGE }\end{array}$ & $\begin{array}{c}\text { PBS } \\
(\mathrm{N}=6)\end{array}$ & $\begin{array}{l}\text { GRFT } \\
(\mathrm{N}=7)\end{array}$ & $\begin{array}{c}\text { PRE-IMM } \\
\text { /GRFT } \\
(\mathrm{N}=7) \\
\end{array}$ & $\begin{array}{c}\text { PASS-IMM / } \\
\text { GRFT } \\
(\mathrm{N}=7)\end{array}$ & $\begin{array}{c}\text { P- } \\
\text { VALUE }\end{array}$ \\
\hline ALB & $\mathrm{g} / \mathrm{dL}$ & $3.8-4.8$ & $5.1 \pm 0.9$ & $5.0 \pm 0.3$ & $4.2 \pm 0.3^{*}$ & $4.5 \pm 0.2$ & 0.0071 \\
\hline ALKP & $U / L$ & $16-302$ & $82 \pm 13$ & $62 \pm 22$ & $97 \pm 33$ & $94 \pm 30$ & 0.0767 \\
\hline ALT & $U / L$ & $20-61$ & $100 \pm 29$ & $74 \pm 19$ & $79 \pm 45$ & $72 \pm 22$ & 0.3717 \\
\hline AMYL & $U / L$ & $326-2246$ & $1336 \pm 155$ & $1577 \pm 236$ & $1554 \pm 180$ & $1681 \pm 190^{*}$ & 0.0468 \\
\hline CA & $\mathrm{mg} / \mathrm{dL}$ & 5.3-11.6 & $12.2 \pm 0.4$ & $12.3 \pm 0.7$ & $12.0 \pm 0.3$ & $12.1 \pm 0.5$ & 0.7027 \\
\hline CHOL & $\mathrm{mg} / \mathrm{dL}$ & 20-92 & $96 \pm 20$ & $116 \pm 15$ & $105 \pm 12$ & $110 \pm 12$ & 0.1292 \\
\hline CREA & $\mathrm{mg} / \mathrm{dL}$ & $0.1-0.6$ & $0.3 \pm 0.1$ & $0.4 \pm 0.1$ & $0.4 \pm 0.0$ & $0.3 \pm 0.1$ & 0.1103 \\
\hline GLU & $\mathrm{mg} / \mathrm{dL}$ & $50-135$ & $194 \pm 53$ & $153 \pm 30$ & $153 \pm 29$ & $151 \pm 44$ & 0.1898 \\
\hline PHOS & $\mathrm{mg} / \mathrm{dL}$ & $5.8-11.2$ & $10.4 \pm 1.8$ & $8.4 \pm 0.9^{*}$ & $8.4 \pm 0.9^{*}$ & $8.3 \pm 0.8^{*}$ & 0.0083 \\
\hline TBIL & $\mathrm{mg} / \mathrm{dL}$ & $0.1-0.7$ & $1.9 \pm 1.8$ & $1.0 \pm 0.6$ & $0.6 \pm 0.2$ & $0.9 \pm 0.8$ & 0.1498 \\
\hline TP & $\mathrm{g} / \mathrm{dL}$ & 5.3-6.9 & $8.7 \pm 1.6$ & $8.4 \pm 0.4$ & $8.0 \pm 0.3$ & $8.4 \pm 0.4$ & 0.4511 \\
\hline BUN & $\mathrm{mg} / \mathrm{dL}$ & $9-21$ & $21 \pm 4$ & $19 \pm 2$ & $23 \pm 2$ & $23 \pm 3$ & 0.0453 \\
\hline GLOB & $\mathrm{g} / \mathrm{dL}$ & $1.5-2.8$ & $3.4 \pm 0.3$ & $3.4 \pm 0.3$ & $3.8 \pm 0.2$ & $3.9 \pm 0.2^{*}$ & 0.002 \\
\hline
\end{tabular}

Table 4.2. Serum Chemistry panels of animals after chronic treatment with PBS or GRFT. Data represent mean values +/- standard deviation for albumin (alb), alkaline phosphatase (ALKP), Alanine Transferase (ALT), amylase (AMYL), calcium (Ca), cholesterol (Chol), Creatinine (Crea), glucose (Glu), phosphorus (Phos), total bilirubin (Tbil), total protein (TP), blood urea nitrogen (BUN), and globulin (Glob). One-way ANOVA with Bonferroni post-test significance versus PBS-treated controls $(p<0.05)$ is indicated by $\left(^{*}\right)$ 


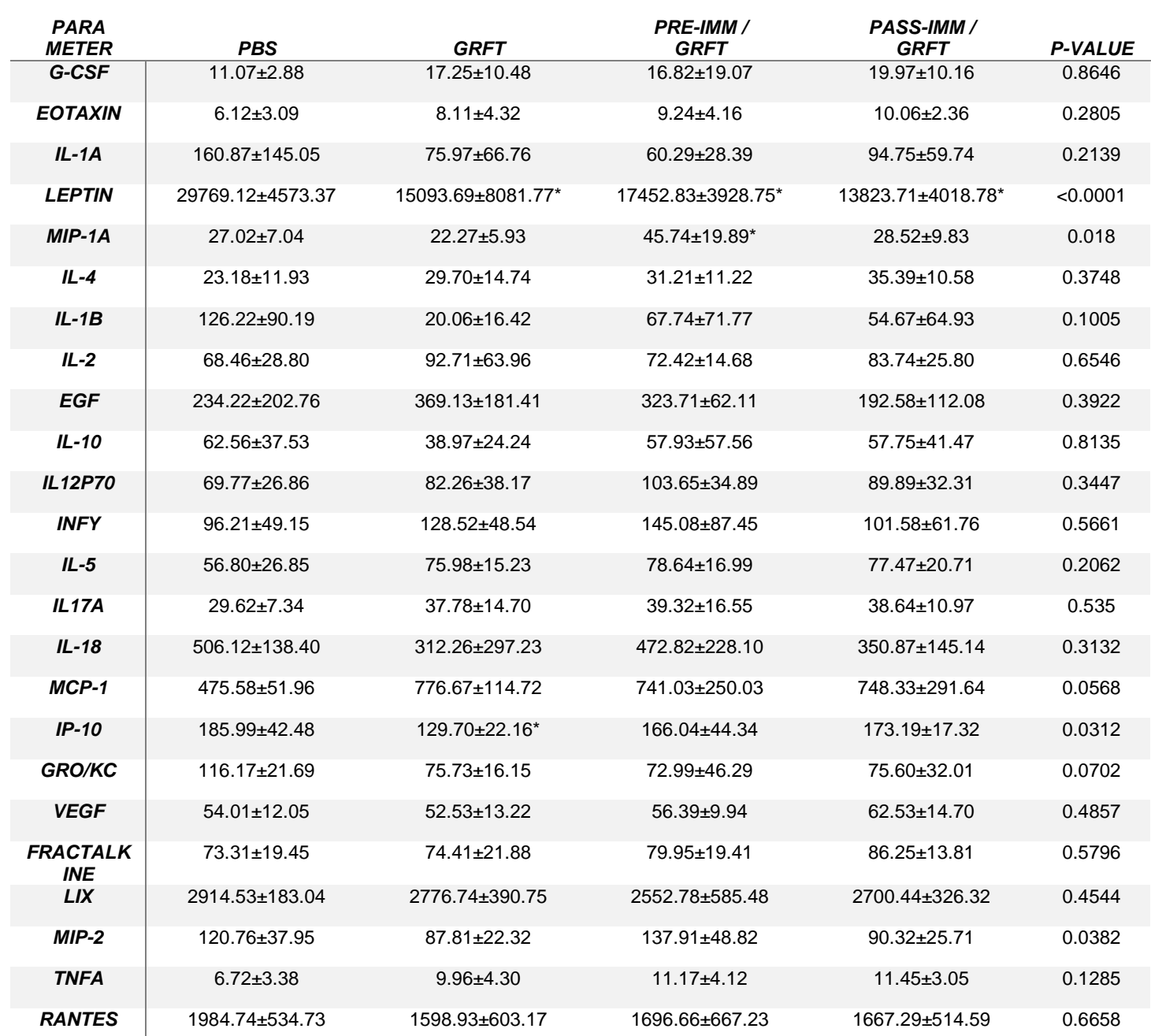

Table 4.3. Cytokine levels after chronic administration with GRFT. Cytokine levels in serum from rats 24 hours after treatment was quantitated. Analytes with three or more positive signals per group: granulocyte colony-stimulating factor (G-CSF), Eotaxin, Granulocyte macrophage colonystimulating factor (GM-SCF), Interleukin 1 alpha (IL-1a), Leptin, Macrophage Inflammatory Protein 1 alpha (MIP 1-a), Interleukin 4 (IL-4), Interleukin 1 beta (IL-1B), Interleukin two (IL-2), EGF, Interleukin 13 (IL-13), Interleukin 10 (IL-10), Interluekin 12 (IL-12p70), Interferon gamma (INFy), Interleukin 5 (IL-5), Interleukin 17 alpha (IL-17A), Interleukin 18 (IL-18), monocyte chemoattractant protein 1 (MCP-1), interferon gamma induced protein 10 (IP-10), growth-related oncogene $(\mathrm{GRO} / \mathrm{KC})$, vascular endothelial growth factor (VEGF), Fractalkine, lipopolysaccharide induced CXC chemokine (LIX), macrophage inflammatory protein 2 (MIP-2), tumor necrosis factor alpha (TNFa), and Rantes. One-way ANOVA with Bonferroni post-test analysis indicating significance vs PBS treated controls $(p<0.05)$ is indicated by $\left(^{*}\right)$. 


\section{CHAPTER 5}

\section{CHARACTERIZATION OF BIOCHEMICAL INTERACTIONS OF GRIFFITHSIN THROUGH DIFFERENTIAL SCANNING FLUORIMETRY, AFFINITY PURIFICATION, AND DIFFERENTIAL SCANNING CALORIMETRY}

\section{INTRODUCTION}

GRFT is a $12.7 \mathrm{kDa}$ carbohydrate binding protein derived from the red algae Griffithsia sp [32]. GRFT displays a strong affinity for N-linked oligomannose glycans $\left(\mathrm{Man}_{5-9} \mathrm{GlcNAc}_{2}\right)$ constituting glycan shields of multiple viruses, making it a strong candidate as an antiviral microbicide and/or therapeutic [163, 164]. Notable antiviral activity has been observed in the case of HIV-1, HCV, Herpes virus, SARS-CoV, and influenza [23, 30, 36, 171, 173]. Given this broad spectrum antiviral activity, research into GRFT's suitability as a systemic antiviral prophylactic is ongoing. Previous in vitro studies have demonstrated that GRFT possesses a favorable toxicity profile, suggesting its viability as a systemic therapeutic. For example, GRFT is neither mitogenic, strongly immunogenic, nor a strong inducer of inflammatory cytokines in human peripheral blood 
mononuclear cells $[35,169]$. Further, previous studies into the impact of in vivo administrations of GRFT have also been promising. GRFT shows in vivo activity against JEV, HCV and SARS-CoV in mouse models [23, 30, 31, 173]. GRFT was also tolerable in a 10-day acute subcutaneous administration study involving both mice and guinea pigs [34].

Recently, we confirmed that GRFT was tolerable over the course of a 55day chronic administration study in rats, with minimal systemic toxicity reported (manuscript in preparation). Interestingly, in prior studies active GRFT concentrations were observable in treated mice and guinea pigs for many days (up to 14 days in mice) after subcutaneous administration[34]. An additional study into GRFT's pharmacokinetics also displayed a rapid elimination or degradation of GRFT following subcutaneous or intravenous administration (manuscript in preparation). However, in that study, active concentrations of GRFT were still detectable at potentially therapeutic levels up to 96 hours following systemic administration. Given GRFTs observed serum persistence and its increasingly recognized potential as a systemic anti-viral therapeutic, further investigation into GRFT's potential binding activity, binding partners, and serum interactions are necessary.

A number of methods exist which can elucidate potential protein-ligand interactions, potential protein-protein interactions, and changes in serum protein interactions, thus allowing full characterization of the protein of interest. These include Differential Scanning Fluorometry, Affinity Purification, and Differential 
Scanning Calorimetry. We utilized these techniques to gain greater insight into GRFT's potential interaction with endogenous proteins and ligands.

Differential scanning fluorimetry (DSF) is useful to measure protein-ligand interactions. DSF has been used to identify potential ligand interactions, including polysaccharide, peptide, and nucleic acid interactions with proteins, in a high-throughput manner [212-217]. Given that ligand binding can increase protein stability, shifts in fluorescence of hydrophobic dyes can be used identify specific binding [214]. In the presence of a ligand, increases in melting temperature $\left(T_{m}\right)$ are observed which can yield data for identifying ligands which may bind to, and stabilize, the protein. Thus DSF can screen for ligand binding interactions which tend to stabilize a protein. Functionally, DSF measures the temperature at which a protein melts through the use of a fluorescent dye, such as SYPRO Orange, which possesses an affinity for hydrophobic regions of a protein [215]. This process, conducted with a PCR instrument in microplate format, provides for a high-throughput manner by which identification of specific protein-ligand interactions can be measured.

Affinity Purification (AP) can identify stable protein-protein interactions. One form, Tandem Affinity Purification has been used successfully to purify proteins for identification by Mass-Spectrometry [218-224]. In another type of AP, a recombinant "bait" protein is used to pull down proteins and protein complexes from biological samples, and these interacting proteins are identified 
by Mass Spectrometry $[225,226]$. While a number of tags have been identified for this process, FLAG tagging of a bait protein has been used in AP with success $[221,223]$.

Finally, differential scanning calorimetry (DSC) is a novel and effective method to explore endogenous protein interactions in serum or plasma. There are over 3000 different proteins in plasma or serum. However, the majority of the proteomic mass consists of 22 proteins, while all the others are in relatively very low abundance [227, 228]. DSC generates an excess specific heat capacity profile, represented as a thermogram, of the most abundant proteins present in plasma or serum[229]. These thermograms represent the weighted average of all the major serum proteins interactions [230]. Alterations in thermograms from a "normal" curve generated from healthy subjects represent changes in major serum protein interactions [231]. The interactome hypothesis holds that low molecular weight proteins or peptide binding to more abundant serum proteins can alter normal serum protein interactions and thermostability [232].

DSC has been used to identify significant differences between the healthy and disease thermograms in conditions such as rheumatoid arthritis, lupus erythematosis, and Lyme disease [229, 230, 233-235]. Furthermore, DSC has been proven to effectively identify changes in thermogram shapes indicative of degrees of disease state in cervical cancer [231]. Therefore, we hypothesized that DSC would be a useful additional tool in determining whether systemic GRFT administration alters endogenous serum protein interactions, which in turn could lead to identification of unseen toxicities associated with GRFT treatment. 
By employing DSF, AP and DSC to study GRFT's potential biochemical interactions, we have confirmed potential binding activity beyond oligomannose moieties, identified a number of putative serum binding partners in human serum, and demonstrated that GRFT spiked into rat serum in vitro does not appear to impact serum protein interactions. However, calorimetric analysis of serum from prior in vivo experiments involving chronic subcutaneous administration of GRFT in rats revealed changes in serum thermograms. These results suggest that protein and/or protein-glycan binding may be a mechanism by which GRFT persists in serum. Further, GRFT by itself does not appear to perturb serum protein interactions. However, it appears that GRFT administration in vivo may induce changes in serum protein binding interactions, though the mechanisms and impact of these interactions will require further study. 


\section{MATERIALS AND METHODS}

Lectin reagents

Recombinant GRFT or GRFT ${ }^{\text {lec- }}$ (a non-binding protein control in which lectin binding sites are eliminated) was produced in Nicotiana benthamiana as described previously, purified to $>99 \%$ purity, and formulated in phosphate buffered saline (PBS), pH 7.4 [169].

\section{Tandem Affinity Purification Lectin reagents}

Linear fusions of GRFT or GRFT ${ }^{\text {lec- }}$ (a non-binding protein control in which lectin binding sites are eliminated) homodimers with a C-terminal FLAG tag and $\mathrm{N}$-terminal $6 \mathrm{xHis}$ tag were cloned by a collaborator (manuscript in preparation) into Icon Genetics vector pICH11599. A three-component vector system was used for expression. Agrobacterium tumefaciens containing the gene of interest, integrase, and cytosolic were infiltrated into 28 d.p.s. (days post sowing) $N$. benthamiana plants. Five days after infiltration, treated $N$. benthamiana plants were harvested and ground in $20 \mathrm{mM}$ sodium phosphate, $500 \mathrm{mM} \mathrm{NaCl}, 20 \mathrm{mM}$ imidazole, ascorbic acid, sodium metabisulfite, $8 \mathrm{M}$ urea, $\mathrm{pH} 7.4$ at $0.5 \mathrm{~g}$ product per $1 \mathrm{ml}$ buffer. The extract was filtered over a 1 filter press and the subsequent cake washed with $25 \%$ volume of buffer. The extract was again filtered through a 0.5 filter press and washed with $25 \%$ volume of buffer. To prepare for chromatography, extracts were filtered through a 0.2 bottle top filter and purified over nickel sepharose GE with a one-step elution gradient to $20 \mathrm{mM}$ sodium phosphate, $500 \mathrm{mM} \mathrm{NaCl}, 250 \mathrm{mM}$ imidazole, $\mathrm{pH}$ 7.4. Elution fractions were 
pooled and diluted 10X with PBS to prepare for FLAG column (Sigma) purification.

\section{DSF reagents}

The lectin binding capacity of GRFT and GRFT ${ }^{\text {lec- }}$ was examined by using SYPRO Orange thermal shift assays. Briefly, SYPRO orange (Sigma) was diluted to $400 x$ concentration in PBS, pH 7.4. GRFT and GRFT ${ }^{\text {lec- }}$ were diluted to $125 \mathrm{uM}$ concentrations. Serial dilutions of the following potential ligands were formulated in PBS: mannose, maltose, glucose, N-acetylglucosamine heparin, mannan, and alpha 1,2 mannobiose.

Samples for protein melt analysis were formulated using $29.05 \mu \mathrm{L}$ of GRFT or GRFT ${ }^{\text {lec- }}, 8.75 \mu \mathrm{L}$ of diluted SYPRO orange, and $32.2 \mu \mathrm{L}$ of ligand dilutions to yield at total volume of $70 \mu \mathrm{L}$ sample. $20 \mu \mathrm{L}$ of each sample were loaded in triplicate into a 96-well PCR plate (NUNC).

Samples were analyzed by an Applied Biosystems StepOnePlus real-time PCR system. Temperature range studied was $20 \mathrm{C}$ to $95^{\circ} \mathrm{C}$, incrementally raised $0.2^{\circ} \mathrm{C} / 15$ seconds. The effect of saccharide binding on protein melting was determined by changes in fluorescent signal as the sample was heated to $95^{\circ} \mathrm{C}$. Controls consisting of highest saccharide concentrations containing no GRFT or $\mathrm{GRFT}^{\text {lec- }}$ were used to determine baseline fluorescence of the ligands, if any. 


\section{Affinity Purification - Human Serum}

Affinity Purification of GRFT-protein complexes was facilitated by use of GRFT or GRFT ${ }^{\text {lec- }}$ containing a His/FLAG tag as a "bait" protein. Briefly, $500 \mu \mathrm{L}$ of Anti-FLAG M2 resin (Sigma) was loaded into $2 \mathrm{ml}$ gravity flow columns (Fisher). Columns were equilibrated by running 5 column volumes of Tris Buffered Saline $(\mathrm{pH} 7.4)$ through the column resin. For bait containing columns, $300 \mu \mathrm{g}$ aliquots of FLAG-GRFT or FLAG-GRFT ${ }^{\text {lec- }}$ protein was then run through respective AntiFLAG gravity columns a total of four times to ensure all FLAG fusion proteins were bound to the resin. A resin-only control column was used to assess nonspecific resin binding.

Human serum (Innovo Research) was diluted five-fold in PBS and run through the column by gravity filtration. After serum was run through the columns, the column resins were washed with 20 column volumes $(40 \mathrm{ml})$ of TBS, $\mathrm{pH}$ 7.4. Isolated proteins were eluted by the addition of $8 \mathrm{~mL}$ of $0.1 \mathrm{M}$ glycine $\mathrm{HCL} \mathrm{pH} \mathrm{3.5,} \mathrm{collected} \mathrm{in} \mathrm{8,} 1 \mathrm{ml}$ fractions. Each $1 \mathrm{ml}$ fraction was collected in Eppendorf tubes containing $10 \mu \mathrm{L}$ of $1 \mathrm{M}$ Tris-HCL pH 9.0. Columns were re-equilibrated by running 10 column volumes of TBS pH 7.4 through the column. Elution fractions were analyzed for protein content by Nanodrop. The highest protein containing fractions were analyzed by Mass Spectrometry.

Proteins in selected elution fractions were identified by ElectroSpray ionization, liquid chromatography and tandem mass spectrometry (Mary Gawinowicz). Briefly, $100 \mu \mathrm{L}$ of sample was combined with $200 \mu \mathrm{L} 8 \mathrm{M}$ urea in $0.1 \mathrm{M}$ Tris- $\mathrm{HCl}, \mathrm{pH}$ 8.5. Diluted sample was then added to the filter unit of a 
Microcon YM-10 cartridge (Millipore) and centrifuged at 14,000 $\mathrm{xg}$ for 10 minutes. $250 \mu \mathrm{L}$ of $8 \mathrm{M}$ urea in $0.1 \mathrm{M}$ Tris $-\mathrm{HCl}, \mathrm{pH} 8.5$, was added to the filtration unit and centrifuged again. The final volume of the sample was $40 \mu \mathrm{L}$. The flow through was discarded. The concentrated sample in the filter unit was diluted with $60 \mu \mathrm{L} 8 \mathrm{M}$ urea in $0.1 \mathrm{M}$ Tris- $\mathrm{HCl}, \mathrm{pH} 8.5$ and $10 \mu \mathrm{L}$ of $0.1 \mathrm{M}$ DTT added. The solution was kept at $60^{\circ} \mathrm{C}$ for $1 \mathrm{~h}$ and then cooled to room temperature. The sample was alkylated by adding $10 \mu \mathrm{L}$ of $0.15 \mathrm{M}$ iodoacetamide. The reaction was allowed to proceed in the dark for 30 minutes. The sample was then diluted with $100 \mu \mathrm{L} 1 \mathrm{M}$ urea in $0.1 \mathrm{M}$ Tris- $\mathrm{HCl}, \mathrm{pH} 8.5$ and centrifuged at $14,000 \mathrm{~g}$ for 10 minutes, followed by another addition of the same buffer and centrifugation at $14,000 \mathrm{~g}$. The process was repeated once. The remaining sample solution in the filter unit was collected and diluted to $50 \mu \mathrm{L}$ with $1 \mathrm{M}$ urea in Tris- $\mathrm{HCl}, \mathrm{pH}$ 8.5. Trypsin (Roche Biochemicals, sequencing grade), $0.2 \mu \mathrm{g}$ in $20 \mu \mathrm{L} 0.1 \mathrm{M}$ Tris- $\mathrm{HCl}$, $\mathrm{pH} 8.5$, was added and the solution incubated at $32^{\circ} \mathrm{C}$ overnight. The digested solution was desalted with a C18 tip and dried in a Speed-Vac concentrator. The digest was redissolved in $20 \mu \mathrm{L} 0.2 \%$ formic acid and $5 \mu \mathrm{L}$ injected onto the LCMS.

LC-MS/MS was conducted on a Waters Q-Tof Ultima hybrid quadrupole/time-of-flight mass spectrometer with a nanoelectrospray source. Capillary voltage was set at $1.8 \mathrm{kV}$ and cone voltage $32 \mathrm{~V}$; collision energy was set according to mass and charge of the ion, from $14 \mathrm{eV}$ to $50 \mathrm{eV}$. Chromatography was performed on an LC Packings HPLC with a C18 PepMap column using a linear acetonitrile gradient with flow rate of $200 \mathrm{nl} / \mathrm{min}$. 
Raw data files were processed using the MassLynx ProteinLynx software and .pkl files were submitted for searching at www.matrixscience.com using the Mascot algorithm.

\section{DSC- Rat Serum Spiking}

For generation of rat serum thermogram profiles after spiking with PBS or GRFT, the protocol for sample preparation and dialysis described by Garbett et al. was used [231]. Briefly, a standard phosphate dialysis buffer (1.7 mM $\mathrm{KH}_{2} \mathrm{PO}_{4}, 8.3 \mathrm{mM} \mathrm{K}_{2} \mathrm{HPO}, 150 \mathrm{mM} \mathrm{NaCl}, 15 \mathrm{mM} \mathrm{Na}_{3} \mathrm{C}_{6} \mathrm{H}_{5} \mathrm{O}_{7}, \mathrm{pH}$ 7.5) was formulated and rat serum was dialyzed for a period of 24 hours at $4^{\circ} \mathrm{C}$ to normalize buffer conditions. Serum was thawed overnight at $4^{\circ} \mathrm{C}$ the night before dialysis. After thawing, $100 \mu \mathrm{L}$ aliquots of rat serum (Sigma) and an aliquot of $100 \mu \mathrm{L}$ of $10.8 \mathrm{mg} / \mathrm{ml}$ GRFT were loaded into Slide-A-Lyzer mini-dialysis units (MWCO 3500; Pierce, Rockford, IL) and dialyzed at $4^{\circ} \mathrm{C}$ against $1 \mathrm{~L}$ of buffer with changes occurring at 3 hours, 7 hours, 11 hours, and overnight. Following dialysis, serum was filtered through a Spin-X centrifuge 0.45 micron centrifuge tube filter and $99 \mu \mathrm{L}$ aliquots were made for spiking with GRFT or PBS. Final dialysis buffer, to serve as a reference and diluent for sample dilutions, was filtered using a $0.2 \mu \mathrm{m}$ rapid flow filter and stored at $4^{\circ} \mathrm{C}$.

Dialyzed GRFT dilutions in PBS were prepared ranging from $10,000 \mathrm{nM}$ to $50 \mathrm{nM}$. Dialyzed serum aliquots were spiked with GRFT or PBS by the addition of $2 \mu \mathrm{L}$ of GRFT dilution or PBS to $98 \mu \mathrm{L}$ of dialyzed serum. The resulting final concentrations ranged from $1 \mathrm{nM}$ to $1000 \mathrm{nM}$, to exceed the upper molarities of 
GRFT concentrations previously observed after subcutaneous dosing in prior studies [34]. Samples were allowed to react at $4^{\circ} \mathrm{C}$ for one hour. Spiked serum samples were diluted in dialysis buffer (1:25) and samples and reference diasylates were loaded into a 96-well plate and loaded into the instrument autosampler thermostatically set at $4^{\circ} \mathrm{C}$.

DSC data were collected with a NanoDSC (TA Instruments). Scans were recorded from $20^{\circ} \mathrm{C}$ to $100^{\circ} \mathrm{C}$ at a scan rate of $1 \mathrm{c} /$ per minute. Duplicate scans were obtained for all samples. Excess specific heat readings for samples and reference buffers were recorded. Baseline corrected scans were obtained by subtracting a suitably adjacent reference buffer-buffer scan from the raw scans. Scans were further normalized for total protein concentration. Total protein concentrations were determined using Pierce bicinchoninic acid protein assay key and modified microplate procedure. Scans were corrected for non-zero baselines using Origin 7 for linear baseline fitting. Interpolated, final thermograms were calculated and average scans calculated from the duplicates were plotted as excess specific heat capacity versus temperature.

\section{DSC - Chronically Dosed Rat Serum}

In a previous study (manuscript in preparation), age-matched Sprague Dawley rats were subcutaneously treated with either PBS or $10 \mathrm{mg} / \mathrm{kg}$ GRFT every three days for 51 days. A group of passively-immunized animals also received $1.4 \mathrm{mg}$ of anti-GRFT Ig formulated in $1 \mathrm{ml}$ of PBS every 14 days. Additionally, one group animals was pre-immunized for 6 weeks prior to the study 
with GRFT and Sigma adjuvant to prime an immune response to GRFT prior to treatment initiation. Blood was drawn via lateral tail vein on the following days:

Day $10,24,38$, and 52 , with sacrifice on day 55 via $\mathrm{CO}_{2}$ asphyxiation followed by cardiac puncture exsanguination. Serum was separated from blood samples following coagulation by centrifugation and stored at $-20^{\circ} \mathrm{C}$ until needed. Given the timing of blood draws in relation to last administration of GRFT or PBS, Bleed 1(day 10) and Bleed 4 (day 52), both 24 hours after administration, were selected for calorimetric analysis.

As described previously, samples were thawed at $4^{\circ} \mathrm{C}$ overnight before dialysis. Samples were dialyzed into standard phosphate buffer for 24 hours with four buffer changes. Following dialysis, serum samples were diluted in dialysis buffer (1:25) and excess specific heat readings at temperature ranges from $20^{\circ} \mathrm{C}$ to $100^{\circ} \mathrm{C}$ were determined. Duplicate runs were completed and average baseline corrected, protein normalized, interpolated values for each sample were generated.

\section{Data Analysis}

Preliminary examination of DSF curves revealed a dynamic range of 45$95^{\circ} \mathrm{C}$. Accordingly scans were truncated to this range for all analysis. Melting transition temperatures $\left(T_{m}\right)$ for each curve was determined using GraphPad 5.01 and Boltzmann Sigmoidal non-linear fit template available at ftp://ftp.sgc.ox.ac.uk/pub/biophysics/GraphPad_templates/. Mean $T_{m}$ values for 
GRFT and GRFT $^{\text {lec- }}$ were determined for pure protein and each ligand concentration.

Proteins recovered by AP were identified by Mascot algorithm. However, not all proteins identified were deemed a reliable "hit". Proteins identified were filtered by both Mascot score and number of "significant" ion matches to increase positive identification confidence. Given that a significant peptide ion match could appear in more than one protein, a minimum of 3 significant $(p<0.05)$ peptide ion matches for a particular protein identified and a minimum Mascot score of 90 were used as thresholds for filtering. Proteins identified were grouped by bait protein used (FLAG-GRFT, FLAG-GRFT ${ }^{\text {lec-}}$, or resin-only).

Preliminary examination of DSC thermograms revealed a dynamic temperature range of 45 to $90^{\circ} \mathrm{C}$ for the serum thermogram curves. Accordingly, scans were truncated to this range for all analysis. Quantitative comparisons of the thermograms were accomplished through calculation of shape and feature metrics as well as selection of points of interest within the dynamic range of the thermogram curve. Parameters considered included: total area under the curve (AUC), maximum curve height $\left(\mathrm{C}_{\mathrm{p}}{ }^{\mathrm{ex}}\right)$, temperature at max height $\left(\mathrm{T}_{\max }\right)$ Curve width at half height (width), maximum peak height at the $60-67 \mathrm{C}$ range $\left(\mathrm{C}_{\mathrm{p}}{ }^{\mathrm{ex}}\right)$, maximum peak height of the secondary peak at the $68-72 \mathrm{C}$ range $\left(\mathrm{C}_{\mathrm{p}}{ }^{\mathrm{ex2}}\right)$, and ratio of the first and second peak amplitudes, and first moment temperature $\left(T_{f m}\right)$. The first moment temperature $\left(T_{f m}\right)$, used to analyze geometric area distribution changes, was calculated as described by Garbett [231]. Additionally, excess specific heat capacities were analyzed for the following temperature points: 55 , 
$60,65,67,70,75$, and $80^{\circ} \mathrm{C}$. Finally, a composite 'similarity metric' developed by a collaborator (Dan Fish) was generated for each curve [234]. This metric is a combination of an average z-scores or p-values for all temperature points, assuming a normal distribution of the control curve, and a correlation measure, allowing a comparison of test curves versus an average control curve. Similarity scores for each curve were generated. Mean similarity scores were then analyzed by one-way ANOVA with Bonferroni posttest analysis to determine which groups were changed, if any.

Thermogram parameters were grouped by treatment group and differences in thermograms were assessed by ANOVA with post-test to identify changes in groups. A p-value of $<0.05$ was deemed significant. Statistical analysis was conducted using GraphPad 5.

For Day 52 thermogram parameters, correlations between certain curve parameters and serum data previously seen for Day 55 terminal bleeds (Barton) of the same animals were evaluated. Pearson Correlation Coefficients and $p$ values were generated using GraphPad 5. 


\section{RESULTS}

$D S F$

Ligand free, protein-only controls consisting of GRFT or GRFT ${ }^{\text {lec- }}$ displayed marked differences in melting profiles, with GRFT's $T_{m}$ at approximately $75.65^{\circ} \mathrm{C}$ and the $\mathrm{T}_{\mathrm{m}}$ for $\mathrm{GRFT}^{\text {lec- }}$ at approximately $80.68^{\circ} \mathrm{C}$ (Figure 5.1, Table 5.1).

Further differences in GRFT and GRFT ${ }^{\text {lec- }}$ fluorescence signals were observed in response to varying ligand concentrations (Figure 2). GRFT displayed substantial shifts in melting temperature in response to $460 \mathrm{mM}$ concentrations of glucose, maltose, mannose, and $\mathrm{N}$-acetyl glucosamine with shifts in $\mathrm{T}_{\mathrm{m}}$ from 9 to $11^{\circ} \mathrm{C}$ (Figure 5.2 and Table 5.1). Furthermore, GRFT displayed shifts of $>1^{\circ} \mathrm{C}$ in melting temperature at $46 \mathrm{mM}$ concentrations of those saccharides as well as $2.3 \mathrm{mg} / \mathrm{ml}$ and $230 \mu \mathrm{g} / \mathrm{ml}$ concentrations of mannan (Figure 5.2B and Table 5.1). In contrast, high concentrations of all ligands

shifted the melting temperatures for $\mathrm{GRFT}^{\text {lec- }}$ by less than $2^{\circ} \mathrm{C}$ (Table 5.1 ). Further, at lower concentrations of ligand, no positive shifts were observed in GRFT $^{\text {lec- }}$.

\section{Affinity Purification}

Purification of human serum proteins using FLAG-GRFT and FLAGGRFT $^{\text {lec- }}$ bait proteins was successful, though the majority of the proteins identified were Ig chains or albumin. MS/MS analysis of the elution fractions revealed a number of proteins were retrieved from human serum. After 
eliminating keratinocyte-related proteins and filtering by Mascot score and $>2$ significant peptide ion hits, a number of candidate binding partners for each purification condition were identified (Table 5.2). Designating and Eliminating proteins bound to resin as nonspecific background revealed proteins/protein complexes interacting with FLAG-GRFT, FLAG-GRFT ${ }^{\text {lec-}}$, as well as both FLAGGRFT and FLAG-GRFT'lec- (Table 5.3).

\section{DSC - GRFT and PBS spiked rat serum}

Rat sera spiked with varying concentrations of GRFT did not display a substantial shifts in thermograms versus PBS-spiked control serum. (Figure 5.3).

\section{DSC- Chronically Treated Rats}

Mean treatment group thermogram curves were generated for bleed 1 (day 10) and bleed 4 (day 52) (Figure 5.4A and B, respectively). Standard deviation of mean group thermograms from both time points were generated for visual inspection. (Figure 5.4C and D) Analysis of thermogram area (Figure 5.5A), max height (Figure 5.5B), $T_{\max }$ (Figure 5.5C), or peak ratios (Figure 5.6C) did not reveal any significant differences between groups on either day 10 or day 52. Further, peak heights were not significantly different among groups at day 10 (Figure 5.6A). However, differences were observed in Width, $C_{p}{ }^{e x 1}$, and $T_{f m}$ for day 52 curves. Day 52 widths were significantly different $(p=0.0266)$, with both pre-immunized and passively immunized animals displaying smaller widths in comparison to PBS-treated controls (Figure 5.5D). $C_{p}{ }^{\text {ex } 1}$ heights in the $60-68^{\circ} \mathrm{C}$ 
range were also significantly different for day 52 samples, with passively immunized animals displaying a significantly decreased $(p=0.0389)$ maximum peak height in that temperature range (Figure 5.6B). Finally, $\mathrm{T}_{\mathrm{fm}}$ were significantly reduced in GRFT treated groups vs. PBS treated controls (Figure $5.6 \mathrm{D}, \mathrm{p}=0.0179)$.

Analysis of group excess specific heat capacities at selected temperatures did not reveal any differences among the groups at day 10 (Figure 5.7A). However, day 52 groups were significantly different at both the $67^{\circ} \mathrm{C}(p=0.03)$ range as well as the $75^{\circ} \mathrm{C}$ temperature $(\mathrm{p}=0.004)$. (Figure 5.7B).

Overall curve similarity scores did not reveal significant differences among treatment groups on day 10, though results did approach significance (Figure 8). However, significant differences between groups were observed on day 52 $(p=0.0069)$. Which? Post-test analysis revealed significant differences in both GRFT and passively-immunized groups in relation to PBS-treated controls.

Pearson correlation analysis among day 52 curve parameters and day 55 serum characteristics revealed both significant positive and negative correlations (Table 5.4). Significant positive correlations were observed between the following parameters (correlation coefficient, p-value): Albumin and Total Protein $(0.85, p<0.0001)$, Area and Width $(0.44, \mathrm{p}=0.0321)$, Area and Max Peak(0.92, $\mathrm{p}<0.0001)$, Globulin and Total Protein $(0.49, \mathrm{p}=0.0189)$, Globulin and Anti-GRFT antibody titers $(0.45, p=0.0375)$, Globulin and $T_{f m}(0.50, p=0.0145)$, and $T_{\max }$ and $T_{f m}(0.52, p=0.0095)$. Significant negative correlations were observed between: 
Albumin and Anti-GRFT antibody titers $(-0.50, \mathrm{p}=0.0188)$, Width and $\mathrm{T}_{\mathrm{fm}}(-0.56$, $\mathrm{p}=0.0041)$, and Pseudovirus neutralization activity and $\mathrm{T}_{\mathrm{fm}}(-0.54, \mathrm{p}=0.0094)$. 


\section{DISCUSSION}

Potential protein and ligand interactions can be of physiological relevance for a new therapeutic. Accordingly, identification of interaction potential can be useful. DSF analysis of both GRFT and GRFT ${ }^{\text {lec- }}$ showed differences in melting points as well as differences in binding of various saccharides. Analysis of fluorescence curves showed that ligand-free GRFT displayed a consistent melting transition temperature at approximately 75 degrees, whereas GRFT ${ }^{\text {lec- }}$ displayed a melting temperature of approximately 80 degrees (Table 5.1). This difference in melting temperature given the modest changes to the protein would suggest that ablation of lectin binding sites result in increased protein stability, an effect also generally theorized to occur when protein binding sites are occupied by ligand $[212,214]$.

Positive shifts in melting temperature as a result of saccharide binding with GRFT and GRFT ${ }^{\text {lec- }}$ were also observed. Ligand binding to a protein's active site will normally increase stability, leading to increase the protein melting temperature. Accordingly it was expected that specific saccharide binding to GRFT's lectin binding sites would result in higher transition temperatures. This was confirmed by the marked increases in melting temperatures of GRFT upon addition of glucose, maltose, mannose, mannan, and $\mathrm{N}$-acetylglucosamine.

While of much lower affinity than oligomannose, interactions between GRFT and these carbohydrates have been observed in other studies [164, 166]. Although GRFT binds strongly to oligomannose glycans [163] via its jacalin-like carbohydrate binding sites, weaker or transient interactions with other 
endogenous saccharide moieties cannot be ruled out and may help to explain GRFT's long serum persistence observed in other studies [34]. Further, similar marked differences in melting temperature was not observed for $\mathrm{GRFT}^{\text {lec- }}$ for all saccharide concentrations. While $\mathrm{GRFT}^{\mathrm{lec}-}$ displayed a higher baseline melting temperature, it only displayed a modest $\left(<2^{\circ} \mathrm{C}\right)$ shifts in melting temperature at the highest saccharide concentrations. This is in stark contrast to the greater $\left(>9^{\circ} \mathrm{C}\right)$ shifts observed in GRFT at comparable concentrations. These data confirm that a range of weaker potential saccharide binding partners for GRFT exist and may be relevant to GRFT's serum stability. Interestingly, high heparin concentrations decreased GRFT's $T_{m}$, suggesting some level of destabilizing effect. However, how and why this destabilization occurs is currently unknown. In addition to interacting with saccharide moieties, GRFT's ability to effectively bind to human serum proteins was confirmed by its interaction in the FLAG-GRFT serum capture columns. While some off-target serum binding was observed in the resin-only serum capture, a number of unique proteins, including lipoprotein and complement proteins, were captured by FLAG-GRFT bait. Further, FLAG-GRFT ${ }^{\text {lec- }}$, also captured a number of proteins, including some that were unique to GRFT $^{\text {lec- }}$. FLAG-GRFT and FLAG-GRFT ${ }^{\text {lec- }}$ differ only in the lectin binding motifs, therefore capture of unique serum proteins by FLAG-GRFT indicates a binding mechanism which requires active lectin binding sites. We hypothesize that those proteins captured by both FLAG-GRFT and FLAGGRFT $^{\text {lec- }}$ may suggest alternative, non-lectin mediated binding mechanisms or binding of the serum proteins to structural motifs on the bait proteins. However, 
the specific nature of each protein's interaction will need further investigation. Additionally, a SwissProt search of post translation modifications typical to the recovered serum proteins also revealed a varying number of $\mathrm{N}$-linked glycans present on the proteins. However, the vast majority of glycans noted were of the $\mathrm{N}$-linked, complex variety. These data, together with saccharide binding data from DSF, indicates that GRFT binds an array of serum proteins, and is not limited to those possessing oligomannose glycans.

Interestingly, a larger percentage of proteins bound exclusively by GRFT were complement proteins and apolipoproteins, attractin, and vitronectin. The mechanism of these binding interactions will need to be confirmed by other binding methods to determine whether they are recovered as a part of a complex, however based upon their exclusive recovery by the FLAG-GRFT column, it appears that they will likely involve the lectin binding sites of GRFT. For instance, the presence of proteins possessing no N-linked glycans, as in the case of Apolipoprotein A-II is suggestive of entire protein complexes being precipitated from serum via this method. Interestingly, despite apparent binding activity with apolipoproteins, cholesterol changes in guinea pigs and rats have not been noted [34] (and manuscript in preparation). Further, given continued antiviral activity observed in serum following systemic administration[34] (and manuscript in preparation), it does not appear that this binding interaction substantially impairs GRFT's antiviral activity. However, complement interactions may be problematic. Complement proteins are important in the innate immune response to pathogens. Accordingly, a binding interaction with these proteins 
could have deleterious effects if it binds in a manner so as to inhibit pathogen recognition or cause functional deficiency of particular complement proteins [236240]. For instance, deficiencies in complement 2 and 4 have been associated with autoimmune diseases, particularly systemic lupus erythematosus [240-242]. Whether these binding interactions cause functional depletion or complement activation will require further study. However, it is entirely possible that this binding activity may be related to the potential immunosuppressive and antiinflammatory effects of GRFT observed in previous long term systemic studies of GRFT in rats.

GRFT's long serum persistence has been observed in our prior studies. It is believed that these protein-protein interactions may be crucial in maintaining a baseline serum concentration of GRFT. Further enhancing these interactions may be useful in exploring GRFT's potential as a long-term systemic preexposure prophylactic.

Calorimetric analysis of spiked rat serum and analysis of samples from a GRFT chronic dosing study in rats also yielded notable results. Spiking large concentrations of GRFT into rat serum did not appreciably change serum thermograms in comparison to the PBS spiked controls (Figure 5.3). This would seem to indicate that GRFT's presence by itself does not disturb major serum protein interactions. However, thermograms of sera from animals chronically treated differed depending on the duration of GRFT treatment (Figure 5.4A). Both visual inspection of mean thermogram curves and statistical analysis consistently showed no significant differences on day 10 other than first moment 
temperatures. Notably, Bonferroni post-test analysis failed to identify which groups were different. However, passively and pre-immunized animals displayed lower mean $\mathrm{T}_{\mathrm{fm}}$ than PBS treated controls. It is hypothesized that these first moment temperature decreases would indicate an increase in overall area at lower temperature ranges, possibly by a greater contribution to area of the mean curve by immunoglobulins (as elevated IgG levels was the aim of preimmunization, and the effect of passive immunization). Further study will be necessary to see if this redistribution of curve area was due to an increase in IgG, IgM, or some other immunoglobulin in those treatment groups.

In stark contrast with day 10 samples, significant changes were induced by day 52 . Visual examination of the mean group thermograms revealed notable differences and statistical analysis of day 52 curve parameters revealed multiple changes in treatment group thermograms vs. PBS-treated controls. Categories such as Width, $\mathrm{C}_{\mathrm{p}}{ }^{\mathrm{ex} 1}$, and $\mathrm{T}_{\mathrm{fm}}$ were significantly different among treatment groups, which could indicate stabilizing protein interactions with major serum proteins, shifting the area of the curve to higher temperatures while narrowing the width of the curve. Stabilization of serum proteins is thought to result in an increased shift of their melting to higher temperatures. A destabilization of endogenous serum protein interactions should result in shifting of thermograms to lower temperatures. Of the serum parameters examined for day 52 samples, GRFT-only treatment appeared to result in a downshift of $T_{\text {fm }}$ not observed in other groups, suggesting a destabilizing alteration of serum protein interactions. Further, the $\mathrm{T}_{\mathrm{fm}}$ did not display a similar shift in pre-immunized or passively 
immunized animals at that time point. Nonetheless, those groups did display changes in width, with passively immunized animals also displaying a decrease in $\mathrm{C}_{p}{ }^{\text {ex1 }}$. Analysis of excess specific heat capacities at specific temperatures revealed few differences, with the only changes seen between Passively Immunized/GRFT-treated animals vs PBS controls at $67^{\circ} \mathrm{C}$ and $75^{\circ} \mathrm{C}$, and a difference between GRFT-treated vs. PBS controls also observed at $75^{\circ} \mathrm{C}$.

The broadest measure of differences between groups appeared to be accomplished by the generation of similarity scores relative to a mean control curve. Day 10 curves differences approached significance, with GRFT-only and GRFT/passively immunized curves displaying the greatest degree of difference versus mean controls. However, by Day 52, the differences are much more substantial. Similarly to day 10, GRFT-only and GRFT/Passively immunized groups displayed very significant changes vs. PBS-treated controls. This difference is not observed in the group which was pre-immunized with GRFT and adjuvant prior to initiation of treatment. Further, in both cases, similarity scores for pre-immunized animals revealed greater similarity to controls than either GRFT or GRFT/passively immunized groups. As such, it would appear that preimmunization or priming of an immune response may blunt some of the alterations in serum protein interactions. Prior studies have suggested that deimmunization of GRFT may be necessary to eliminate some of the changes induced by systemic administration of GRFT [34, 161, 162]. However, these data suggest that an immune response to GRFT may be protective of the changes induced by systemic administration of the protein. 
Finally correlation analysis between selected day 52 curve parameters and serum parameters from the same animals bled at day 55 revealed a number of significant positive and negative correlations. These correlations assumed non-rapid alteration of serum protein interactions over the intervening 3 day period, and were necessary given exhaustion of day 52 and day 55 serum for analysis. In many instances, correlations between total globulins, albumin, and total protein were expected. Further, these correlations revealed increases in globulin positively correlated with increased in $\mathrm{T}_{\mathrm{fm}}$. However, no curve parameter significantly correlated with anti-GRFT activity determined by antibody titer in a previous study.

In conclusion, DSF and AP confirms that GRFT is capable of binding saccharide moieties and proteins beyond those displaying high-mannose (oligomannose) glycans [164, 166]. Further, in some instances, it appears that GRFT may use lectin-binding sites to bind to endogenous serum proteins, potentially through binding to other types of N-linked glycans. However, it is also wholly possible that in some instances, GRFT is the "prey", given the number of proteins captured by both GRFT and GRFT ${ }^{\text {lec- }}$. These interactions may prove to be the foundation of GRFT's long serum persistence and further study may yield insights into how GRFT's serum persistence could be extended without the sacrifice of anti-viral activity. Additionally, DSC results have shown that while chronic GRFT administration has been observed as tolerable in other studies, alterations to endogenous serum protein interactions may result as a consequence of longer-term chronic treatment. However, these alterations do 
not appear to be due to the simple addition of GRFT into the system. These results would indicate that GRFT administration results in an induction/suppression of other proteins, alterations in the binding of lower molecular weight proteins to the more abundant proteins, or alteration of higher abundance protein proportions, to which DSC is extremely sensitive. Of note, while still altered to a degree, animals pre-immunized with GRFT and adjuvant consistently displayed less thermogram variation in contrast to GRFT-only or GRFT/passively immunized treatment groups. This would seem to suggest a protective immunological response, beyond simple production of immunoglobulins. Further, based on prior findings, that response does not appear to compromise antiviral activity. However, further study into exactly how these changes are induced, as well as which proteins interactions are altered, will need further study. 


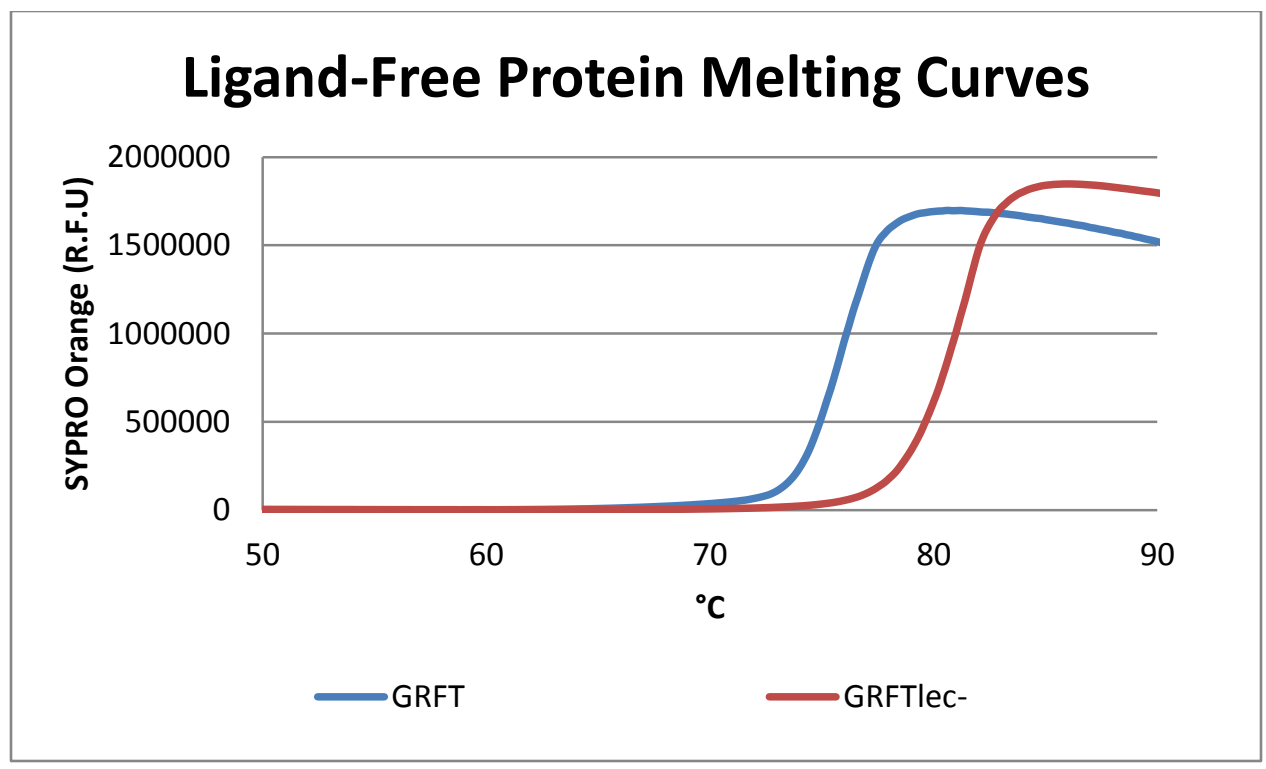

Figure 5.1. GRFT and GRFT ${ }^{\text {lec- }}$ SYPRO orange denaturation curves in the absence of saccharide ligand. Ligand-free fluorescence curves generated by thermal denaturation of GRFT or $\mathrm{GRFT}^{\mathrm{lec}-}$ in the presence of PBS and SYPRO Orange. 


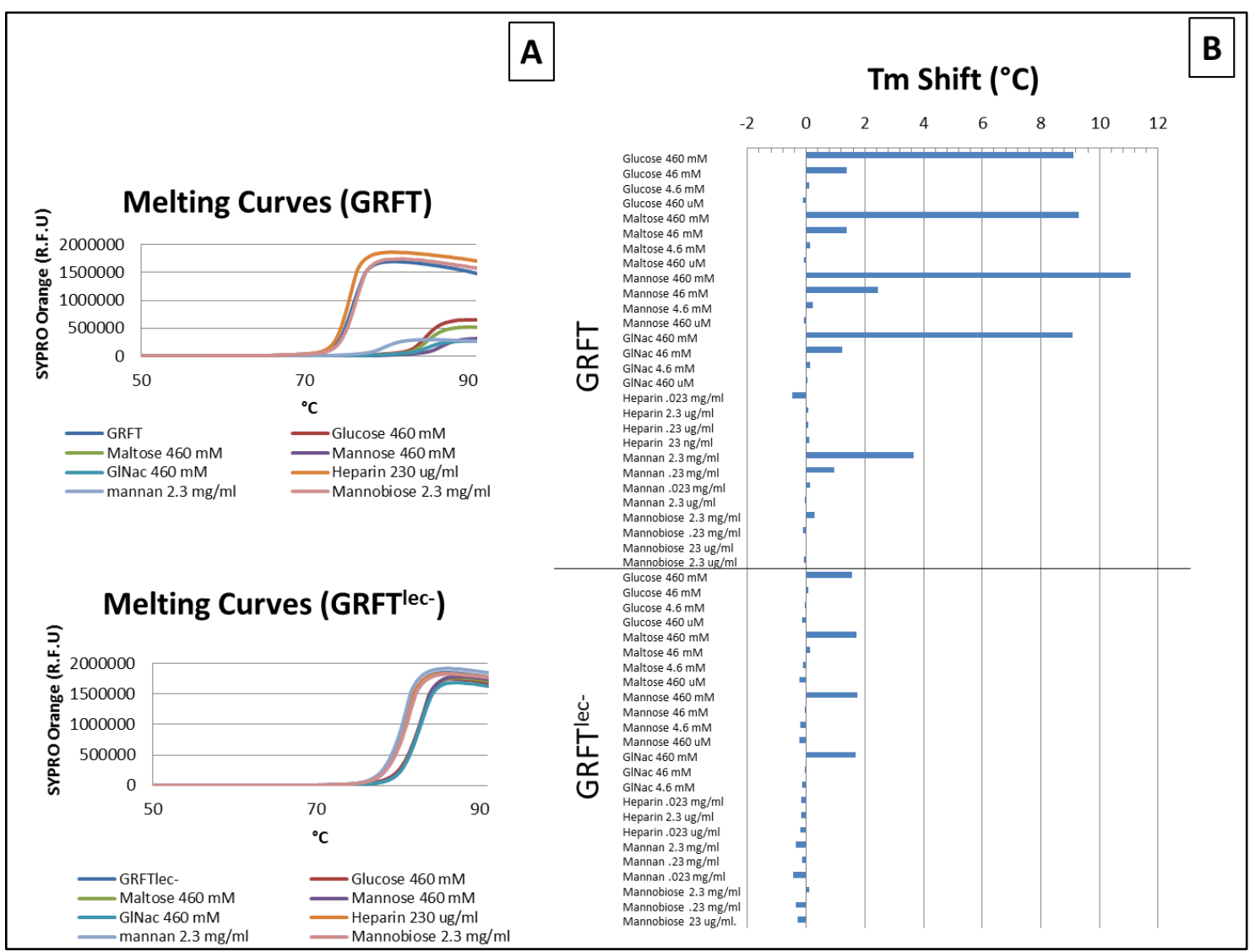

Figure 5.2. Protein stabilization effects of ligand binding on GRFT and GRFT ${ }^{\text {lec- }}$. Fluorescence curves of GRFT and GRFT ${ }^{\text {lec- }}$ in the presence of potential ligands (A) and shifts in melting temperatures $\left(T_{m}\right)(B)$. 


\begin{tabular}{|c|c|c|c|c|}
\hline Protein/Ligand & $\begin{array}{c}\text { Ligand } \\
\text { Concentration } \\
\end{array}$ & $\begin{array}{c}\text { Mean } \\
\text { Tm }\end{array}$ & $\frac{\text { Std. }}{\underline{\text { Dev. }}}$ & $\stackrel{\text { Tm }}{\text { Change }}$ \\
\hline GRFT & - & 75.65 & 0.04 & - \\
\hline \multirow{4}{*}{ Glucose } & $460 \mathrm{mM}$ & 84.74 & 0.02 & 9.09 \\
\hline & $46 \mathrm{mM}$ & 77.02 & 0.01 & 1.37 \\
\hline & $4.6 \mathrm{mM}$ & 75.76 & 0.04 & 0.11 \\
\hline & $460 \mu \mathrm{M}$ & 75.55 & 0.05 & -0.10 \\
\hline \multirow{4}{*}{ Maltose } & $460 \mathrm{mM}$ & 84.93 & 0.01 & 9.28 \\
\hline & $46 \mathrm{mM}$ & 77.02 & 0.01 & 1.37 \\
\hline & $4.6 \mathrm{mM}$ & 75.77 & 0.04 & 0.12 \\
\hline & $460 \mu \mathrm{M}$ & 75.56 & 0.05 & -0.09 \\
\hline \multirow{4}{*}{ Mannose } & $460 \mathrm{mM}$ & 86.70 & 0.06 & 11.05 \\
\hline & $46 \mathrm{mM}$ & 78.08 & 0.01 & 2.43 \\
\hline & $4.6 \mathrm{mM}$ & 75.87 & 0.08 & 0.22 \\
\hline & $460 \mu \mathrm{M}$ & 75.56 & 0.05 & -0.09 \\
\hline \multirow{4}{*}{ GINac } & $460 \mathrm{mM}$ & 84.74 & 0.01 & 9.09 \\
\hline & $46 \mathrm{mM}$ & 76.88 & 0.02 & 1.23 \\
\hline & $4.6 \mathrm{mM}$ & 75.77 & 0.05 & 0.12 \\
\hline & $460 \mu \mathrm{M}$ & 75.67 & 0.03 & 0.02 \\
\hline \multirow{4}{*}{ Heparin } & $23 \mu \mathrm{g} / \mathrm{ml}$ & 75.18 & 0.04 & -0.47 \\
\hline & $2.3 \mu \mathrm{g} / \mathrm{ml}$ & 75.71 & 0.01 & 0.06 \\
\hline & $0.23 \mu \mathrm{g} / \mathrm{ml}$ & 75.71 & 0.04 & 0.06 \\
\hline & $23 \mathrm{ng} / \mathrm{ml}$ & 75.75 & 0.04 & 0.10 \\
\hline \multirow{4}{*}{ Mannan } & $2.3 \mathrm{mg} / \mathrm{ml}$ & 79.38 & 0.06 & 3.73 \\
\hline & $0.23 \mathrm{mg} / \mathrm{ml}$ & 76.86 & 0.59 & 1.21 \\
\hline & $23 \mu \mathrm{g} / \mathrm{ml}$ & 75.79 & 0.04 & 0.14 \\
\hline & $2.3 \mu \mathrm{g} / \mathrm{ml}$ & 75.61 & 0.04 & -0.04 \\
\hline \multirow{4}{*}{ Mannobiose } & $2.3 \mathrm{mg} / \mathrm{ml}$ & 75.92 & 0.02 & 0.27 \\
\hline & $0.23 \mathrm{mg} / \mathrm{ml}$ & 75.53 & 0.07 & -0.12 \\
\hline & $23 \mu \mathrm{g} / \mathrm{ml}$ & 75.63 & 0.05 & -0.02 \\
\hline & $2.3 \mu \mathrm{g} / \mathrm{ml}$ & 75.57 & 0.05 & -0.08 \\
\hline
\end{tabular}

\begin{tabular}{|c|c|c|c|c|}
\hline Protein/Ligand & $\begin{array}{c}\text { Ligand } \\
\text { Concentration } \\
\end{array}$ & $\begin{array}{c}\text { Mean } \\
\text { Im }\end{array}$ & $\begin{array}{l}\text { Std. } \\
\text { Dev. }\end{array}$ & $\underset{\text { Change }}{\stackrel{\text { Tm }}{\text { Chand }}}$ \\
\hline GRFTlec- & - & 80.68 & 0.04 & - \\
\hline \multirow{4}{*}{ Glucose } & $460 \mathrm{mM}$ & 82.26 & 0.03 & 1.58 \\
\hline & $46 \mathrm{mM}$ & 80.75 & 0.02 & 0.07 \\
\hline & $4.6 \mathrm{mM}$ & 80.63 & 0.12 & -0.05 \\
\hline & $460 \mu \mathrm{M}$ & 80.53 & 0.09 & -0.15 \\
\hline \multirow{4}{*}{ Maltose } & $460 \mathrm{mM}$ & 82.40 & 0.01 & 1.72 \\
\hline & $46 \mathrm{mM}$ & 80.81 & 0.08 & 0.13 \\
\hline & $4.6 \mathrm{mM}$ & 80.57 & 0.07 & -0.11 \\
\hline & $460 \mu \mathrm{M}$ & 80.45 & 0.08 & -0.23 \\
\hline \multirow{4}{*}{ Mannose } & $460 \mathrm{mM}$ & 82.43 & 0.05 & 1.75 \\
\hline & $46 \mathrm{mM}$ & 80.63 & 0.06 & -0.05 \\
\hline & $4.6 \mathrm{mM}$ & 80.48 & 0.08 & -0.20 \\
\hline & $460 \mu \mathrm{M}$ & 80.46 & 0.06 & -0.22 \\
\hline \multirow{3}{*}{ GINac } & $460 \mathrm{mM}$ & 82.36 & 0.04 & 1.68 \\
\hline & $46 \mathrm{mM}$ & 80.64 & 0.01 & -0.04 \\
\hline & $4.6 \mathrm{mM}$ & 80.54 & 0.08 & -0.14 \\
\hline \multirow{3}{*}{ Heparin } & $23 \mu \mathrm{g} / \mathrm{ml}$ & 80.53 & 0.09 & -0.15 \\
\hline & $2.3 \mu \mathrm{g} / \mathrm{ml}$ & 80.53 & 0.05 & -0.15 \\
\hline & $0.23 \mu \mathrm{g} / \mathrm{ml}$ & 80.47 & 0.05 & -0.21 \\
\hline \multirow{3}{*}{ Mannan } & $2.3 \mathrm{mg} / \mathrm{ml}$ & 80.34 & 0.06 & -0.34 \\
\hline & $0.23 \mathrm{mg} / \mathrm{ml}$ & 80.55 & 0.05 & -0.13 \\
\hline & $23 \mu \mathrm{g} / \mathrm{ml}$ & 80.25 & 0.02 & -0.43 \\
\hline \multirow{3}{*}{ Mannobiose } & $2.3 \mathrm{mg} / \mathrm{ml}$ & 80.77 & 0.03 & 0.09 \\
\hline & $0.23 \mathrm{mg} / \mathrm{ml}$ & 80.33 & 0.06 & -0.35 \\
\hline & $23 \mu \mathrm{g} / \mathrm{ml}$ & 80.41 & 0.02 & -0.27 \\
\hline
\end{tabular}

Table 5.1. Concentration dependent shifts in melting temperatures of GRFT and GRFT $^{\text {lec- }}$ determined by SYPRO orange fluorescence shifts. $T_{m}$ values, representing melting temperatures for GRFT and $\mathrm{GRFT}^{\text {lec- }}$ were determined in the absence and presence of ligands. $T_{m}$ change values were calculated for all ligand concentrations by subtracting the Tm for GRFT or $\mathrm{GRFT}^{\mathrm{lec}}$ - in the absence of ligand. 


\begin{tabular}{|c|c|c|}
\hline \multicolumn{3}{|c|}{ Proteins Recovered (>2Significant Ions Identified) } \\
\hline$\underline{\text { Resin-Only }}$ & FLAG-GRFT & $\underline{\text { FLAG-GRFTlec- }}$ \\
\hline CD5 antigen-like & Alpha-1-antitrypsin & Alpha-1-antitrypsin \\
\hline Histidine-rich glycoprotein & Apolipoprotein A-I & Alpha-2-macroglobulin \\
\hline Ig alpha- 1 chain $C$ region & Apolipoprotein A-II & Apolipoprotein B-100 \\
\hline Ig alpha- 2 chain $C$ region & Apolipoprotein B-100 & C4b-binding protein alpha chain \\
\hline Ig gamma- 1 chain $\mathrm{C}$ region & Apolipoprotein D & CD5 antigen-like \\
\hline Ig gamma- 2 chain $C$ region & Attractin & Complement $\mathrm{C} 3$ \\
\hline Ig gamma- 3 chain $\mathrm{C}$ region & C4b-binding protein alpha chain & Complement factor $\mathrm{H}$ \\
\hline Ig heavy chain V-III region WEA & CD5 antigen-like & Haptoglobin \\
\hline Ig kappa chain C region & Complement $\mathrm{C} 2$ & Haptoglobin-related protein \\
\hline Ig lambda- 1 chain $C$ regions & Complement C3 & Ig alpha- 1 chain $C$ region \\
\hline Ig lambda-2 chain C regions & Complement C4-A & Ig alpha- 2 chain $C$ region \\
\hline Ig mu chain $\mathrm{C}$ region & Complement factor $\mathrm{H}$ & Ig gamma- 1 chain $C$ region \\
\hline Immunoglobulin J chain & Haptoglobin & Ig gamma- 2 chain $\mathrm{C}$ region \\
\hline Keratin, type I cytoskeletal 10 & Haptoglobin-related protein & Ig gamma-3 chain $\mathrm{C}$ region \\
\hline Keratin, type I cytoskeletal 9 & Histidine-rich glycoprotein & Ig gamma- 4 chain $\mathrm{C}$ region \\
\hline \multirow[t]{13}{*}{ Serum albumin } & Ig alpha- 1 chain $C$ region & Ig kappa chain C region \\
\hline & Ig alpha- 2 chain $\mathrm{C}$ region & Ig lambda-2 chain C regions \\
\hline & Ig gamma- 1 chain $\mathrm{C}$ region & Ig mu chain $\mathrm{C}$ region \\
\hline & Ig gamma- 2 chain $\mathrm{C}$ region & Ig mu heavy chain disease protein \\
\hline & Ig gamma- 3 chain $\mathrm{C}$ region & Immunoglobulin J chain \\
\hline & Ig kappa chain C region & $\begin{array}{l}\text { Immunoglobulin lambda-like } \\
\text { polypeptide }\end{array}$ \\
\hline & Ig lambda-2 chain C regions & Keratin, type I cytoskeletal \\
\hline & Ig mu chain $\mathrm{C}$ region & Serotransferrin \\
\hline & Ig mu heavy chain disease protein & Serum albumin \\
\hline & Immunoglobulin J chain & \\
\hline & $\begin{array}{l}\text { Immunoglobulin lambda-like } \\
\text { polypeptide5 }\end{array}$ & \\
\hline & Serum albumin & \\
\hline & Vitronectin & \\
\hline
\end{tabular}

Table 5.2. Proteins recovered from Human Serum by Affinity Purification with

FLAG-fusion protein. Proteins recovered by affinity purification with Anti-Flag resin, FLAG-GRFT, or FLAG-GRFT ${ }^{\text {lec- }}$ were identified by tandem mass spectrometry and filtered by MASCOT score and $>2$ significant peptide ion hits. 


\begin{tabular}{|c|c|c|c|c|c|}
\hline FLAG-GRFT & $\begin{array}{l}\text { Gly. } \\
\text { Sites }\end{array}$ & FLAG-GRFT' & $\frac{\text { Gly. }}{\text { Sites }}$ & $\begin{array}{l}\text { FLAG-GRFT and } \\
\text { FLAG-GRFTlec- }\end{array}$ & $\begin{array}{l}\text { Gly. } \\
\text { Sites }\end{array}$ \\
\hline $\begin{array}{c}\text { Apolipoprotein } \\
\text { A-I }\end{array}$ & $\begin{array}{c}\overline{1 \mathrm{~N}} \\
\text { (glycation) }\end{array}$ & $\begin{array}{c}\text { Alpha-2- } \\
\text { macroglobulin }\end{array}$ & $8 \mathrm{~N}$ & Alpha-1-antitrypsin & $\overline{3 N}$ \\
\hline $\begin{array}{c}\text { Apolipoprotein } \\
\text { A-II }\end{array}$ & 0 & $\begin{array}{c}\text { Ig gamma-4 chain } \mathrm{C} \\
\text { region }\end{array}$ & $1 \mathrm{~N}$ & $\begin{array}{c}\text { Apolipoprotein B- } \\
100\end{array}$ & $19 N$ \\
\hline Apolipoprotein D & $2 \mathrm{~N}$ & Serotransferrin & $3 \mathrm{~N}, 1 \mathrm{O}$ & $\begin{array}{l}\text { C4b-binding protein } \\
\text { alpha chain }\end{array}$ & $3 N$ \\
\hline Attractin & $26 \mathrm{~N}$ & & & Complement C3 & $3 N$ \\
\hline Complement C2 & $8 \mathrm{~N}$ & & & $\begin{array}{c}\text { Complement factor } \\
\mathrm{H}\end{array}$ & $9 \mathrm{~N}$ \\
\hline $\begin{array}{l}\text { Complement } \\
\text { C4-A }\end{array}$ & $4 \mathrm{~N}, 1 \mathrm{O}$ & & & Haptoglobin & $4 \mathrm{~N}$ \\
\hline \multirow[t]{3}{*}{ Vitronectin } & $3 N$ & & & $\begin{array}{l}\text { Haptoglobin-related } \\
\text { protein }\end{array}$ & 0 \\
\hline & & & & $\begin{array}{l}\text { Ig mu heavy chain } \\
\text { disease protein }\end{array}$ & $1 \mathrm{~N}$ \\
\hline & & & & $\begin{array}{l}\text { Immunoglobulin } \\
\text { lambda-like } \\
\text { polypeptide } 5\end{array}$ & 0 \\
\hline
\end{tabular}

Table 5.3. Unique proteins identified by affinity purification. Proteins were recovered by affinity purification using Anti-FLAG resin, FLAG-GRFT, and FLAGGRFT. After filtering by Mascot score and $>2$ significant peptide ion hits, background binding was determined by proteins binding to Anti-FLAG resin-only controls. After elimination of proteins non-specifically binding to Anti-FLAG resin, a number of proteins unique to FLAG-GRFT or FLAG-GRFT ${ }^{\text {lec- }}$ were identified. Glycosylation state was determined by SwissProt search. 


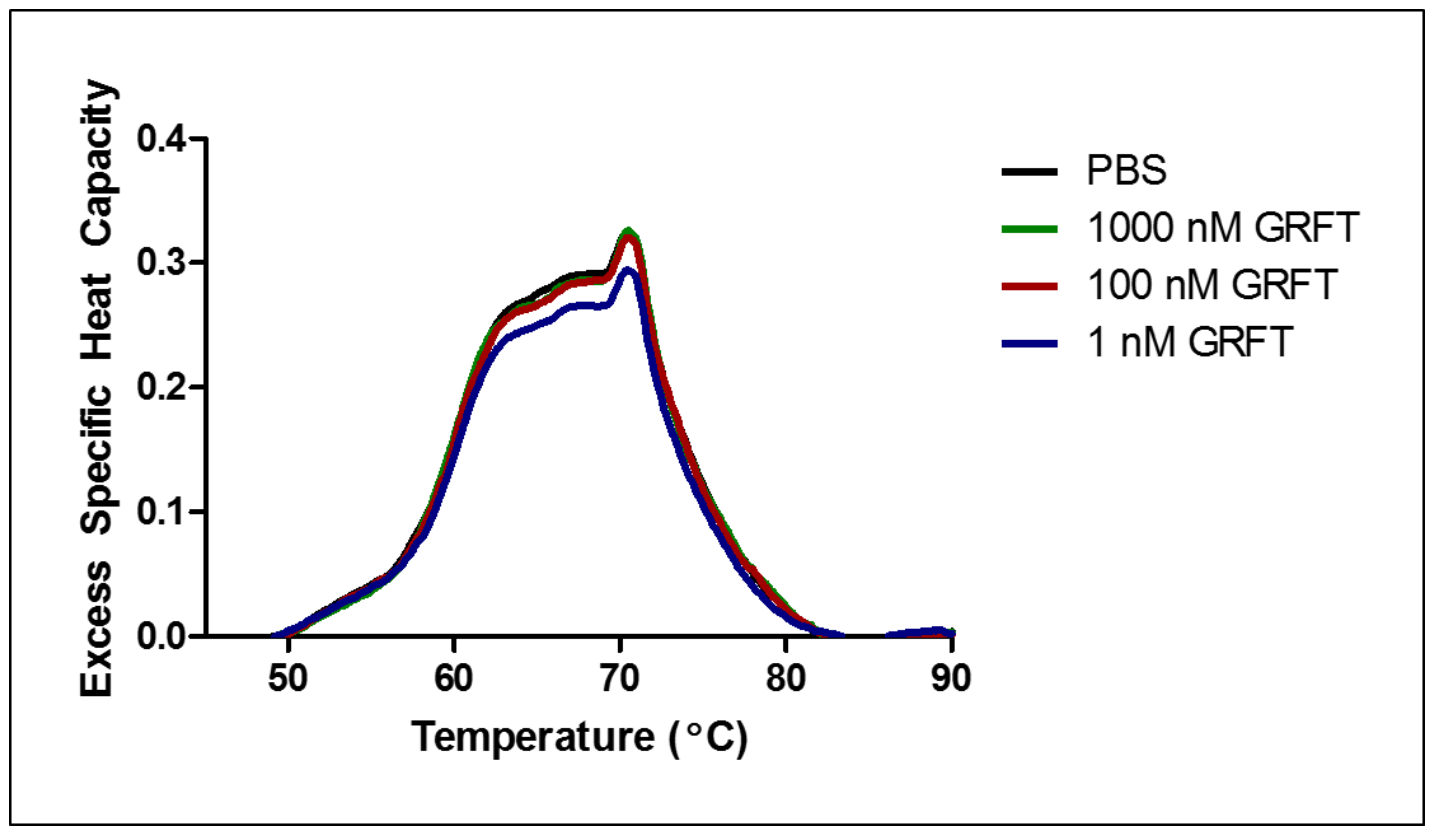

Figure 5.3. Thermogram profiles of rat serum spiked with varying concentrations of GRFT. Thermogram profiles of excess specific heat capacity versus temperature for rat serum spiked with a range of concentrations of GRFT. Concentrations were final molarity concentrations of GRFT spiked into rat serum before $25 x$ dilution of samples for calorimetric analysis. 


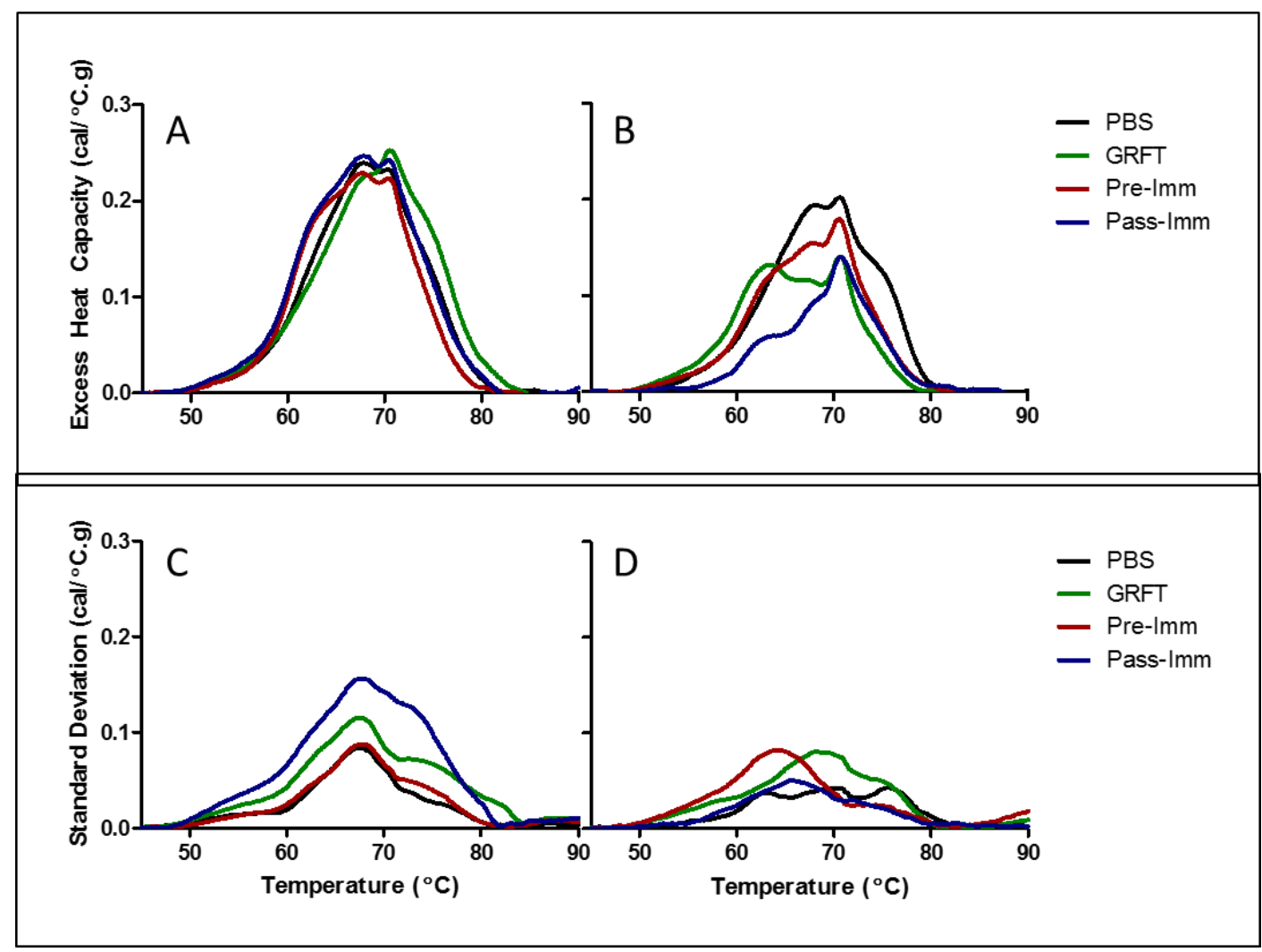

Figure 5.4. Mean thermograms generated from sera from rats chronically treated with Griffithsin or PBS. Mean thermogram profiles of excess specific heat capacity (cal/ $\left.{ }^{\circ} \mathrm{C} . \mathrm{g}\right)$ versus temperature for Day $10(\mathrm{~A})$ and Day $52(\mathrm{~B})$ serum samples from animals chronically treated with PBS, GRFT, PreImmunized/GRFT-treated [Pre-imm], or Passively Immunized/GRFT-treated [Pass-Imm]. Standard Deviation of excess specific heat capacity of each group for day $10(C)$ and day 52 (D) was generated for comparison. 


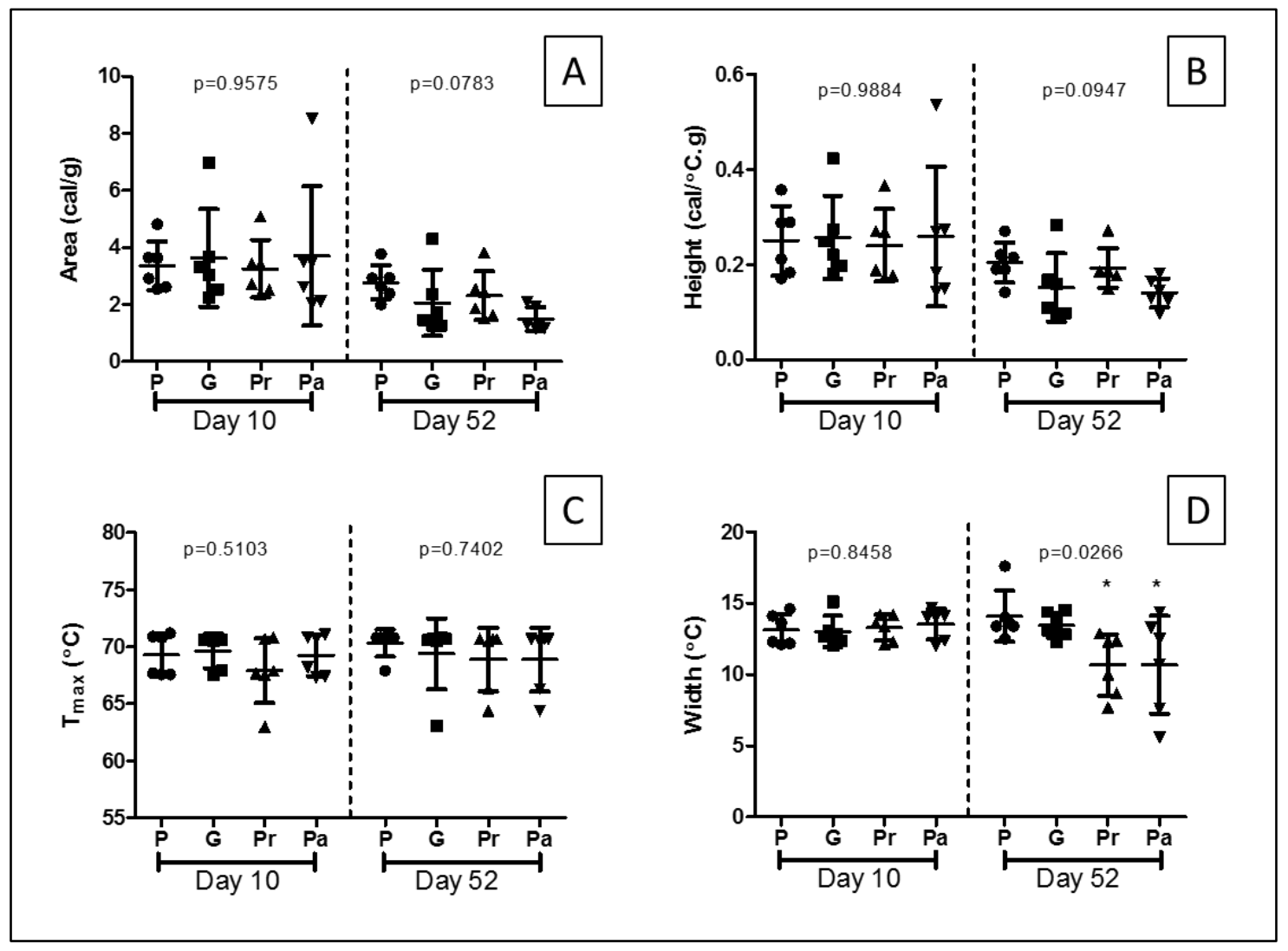

Figure 5.5. Thermogram shape and feature parameters for rats chronically treated with GRFT or PBS. Values for Area (A), Height (B), Temperature at max height (C), and curve width (D) were calculated for each thermogram generated from serum from PBS [P], GRFT [G], Pre-immunized/GRFT-treated [Pr] and Passively-immunized/GRFT-treated $[\mathrm{Pa}]$ animals. Mean values for treatment groups determined for treatment day 10 and 52 were analyzed by one-way ANOVA with Bonferroni posttest. Bars represent mean and standard deviation of six biological replicates. A p-value of $<0.05$ was deemed significant $\left({ }^{*}\right)$. 

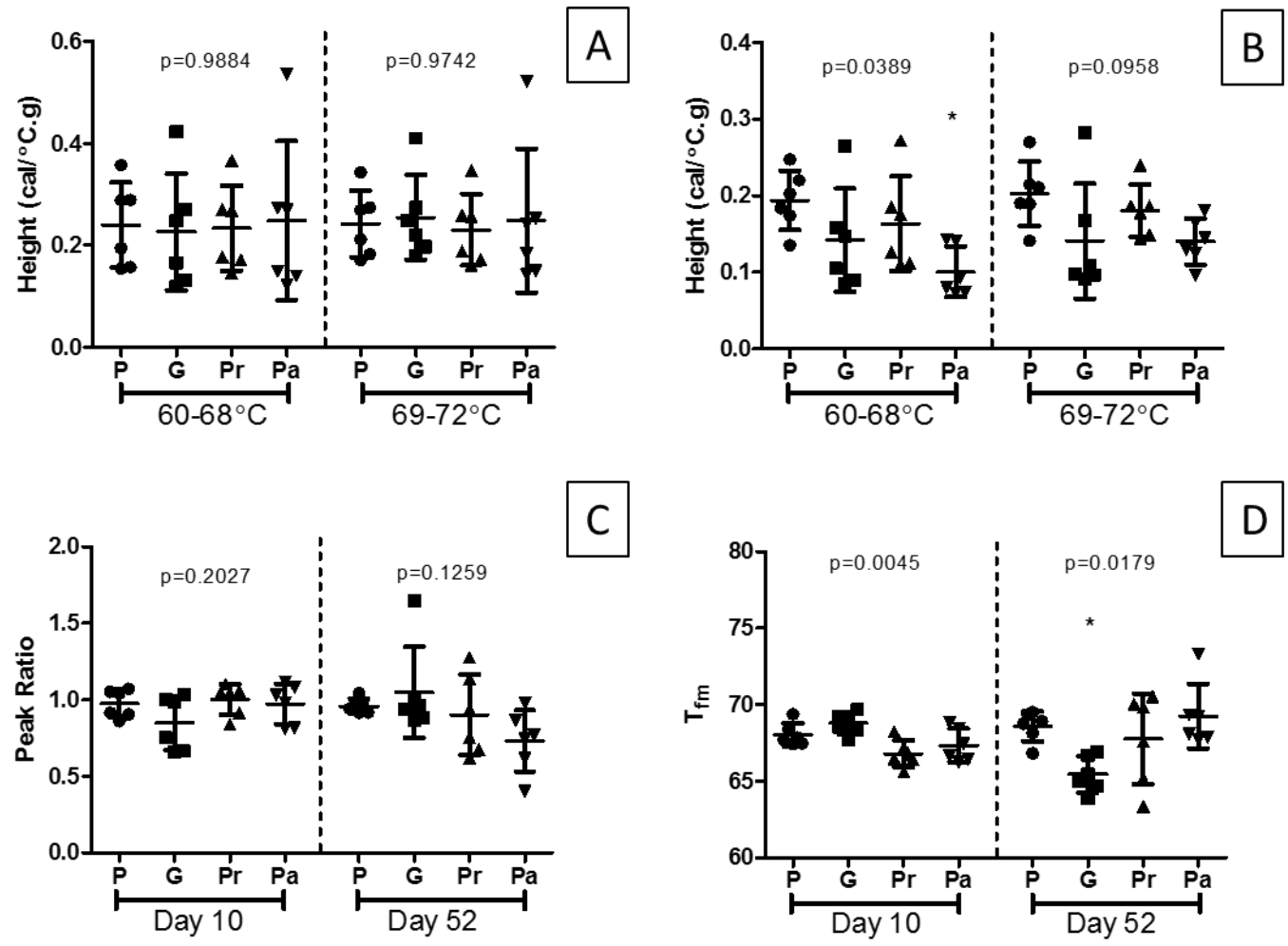

C

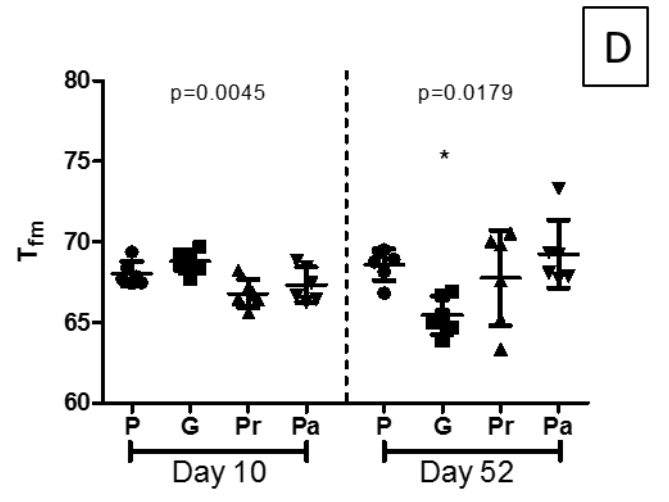

Figure 5.6. Peak and First-moment $\left(T_{f m}\right)$ thermogram parameters for rats chronically treated with GRFT or PBS. Values for Peak Heights on day 10 (A), Peak heights on day 52 (B), Peak Ratios (C), and first moment temperatures (D) were calculated for each thermogram generated from serum from PBS [P], GRFT [G], Pre-immunized/GRFT-treated [Pr] and Passively-immunized/GRFT-treated $[\mathrm{Pa}]$ animals. Mean values for treatment groups determined for treatment day 10 and 52 were analyzed by one-way ANOVA with Bonferroni posttest. Bars represent mean plus standard deviation of six biological replicates. A p-value of $<0.05$ was deemed significant $\left(^{*}\right)$. 


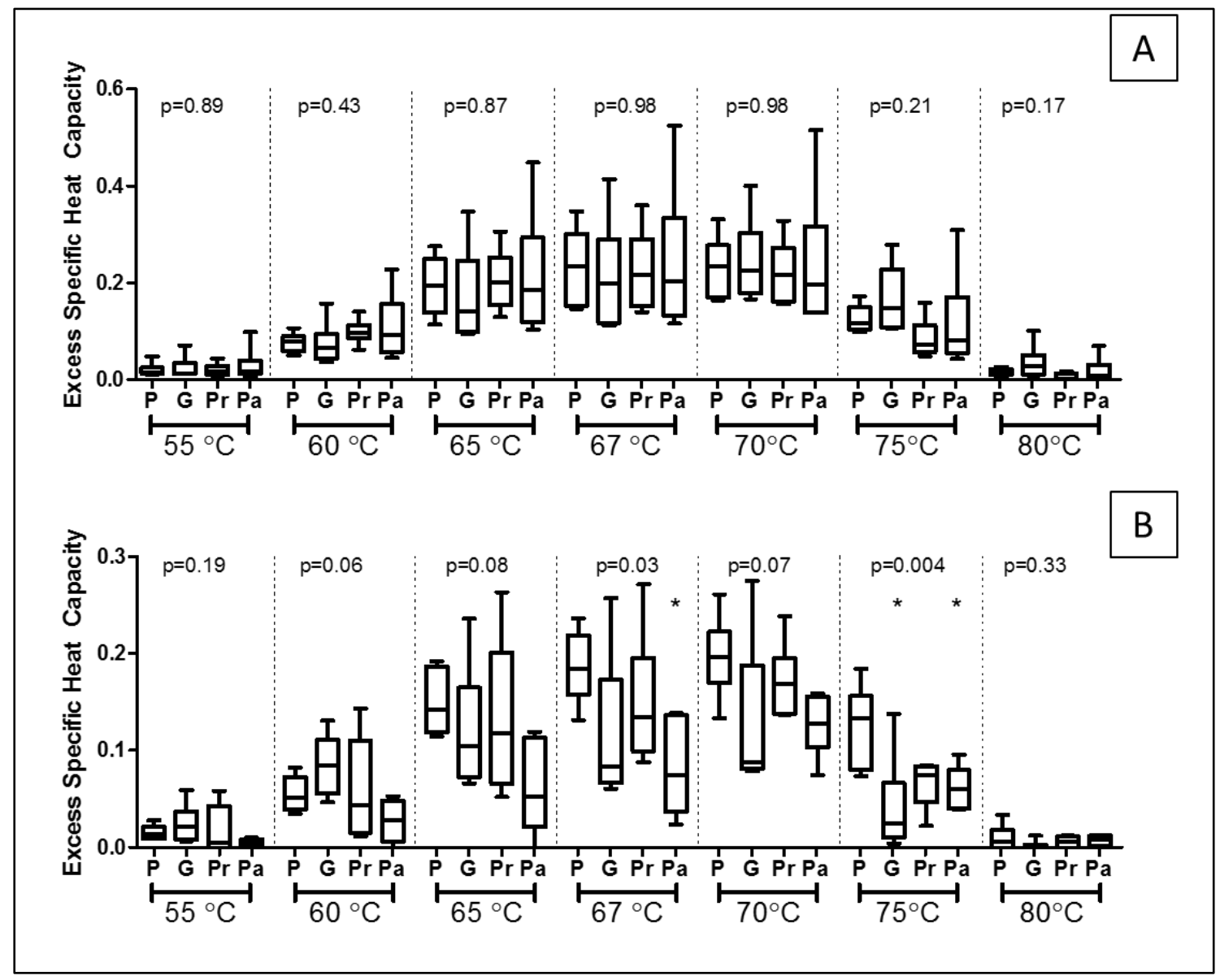

Figure 5.7. Distribution of Excess specific heat capacity for rats chronically treated with GRFT or PBS at select temperature points. Excess specific heat capacities on day $10(\mathrm{~A})$ and day $52(\mathrm{~B})$ for animals treated with PBS [P], GRFT [G], Pre-immunized/GRFT-treated [Pr] and Passively-immunized/GRFT-treated [Pa] at $55,60,65,67,70,75$, and $80^{\circ} \mathrm{C}$ were determined. Whiskers indicate Maximum and minimum values. Mean values were analyzed by one-way ANOVA with Bonferroni posttest. A $p$-value of $p<0.05$ was considered significant $\left({ }^{*}\right)$. 


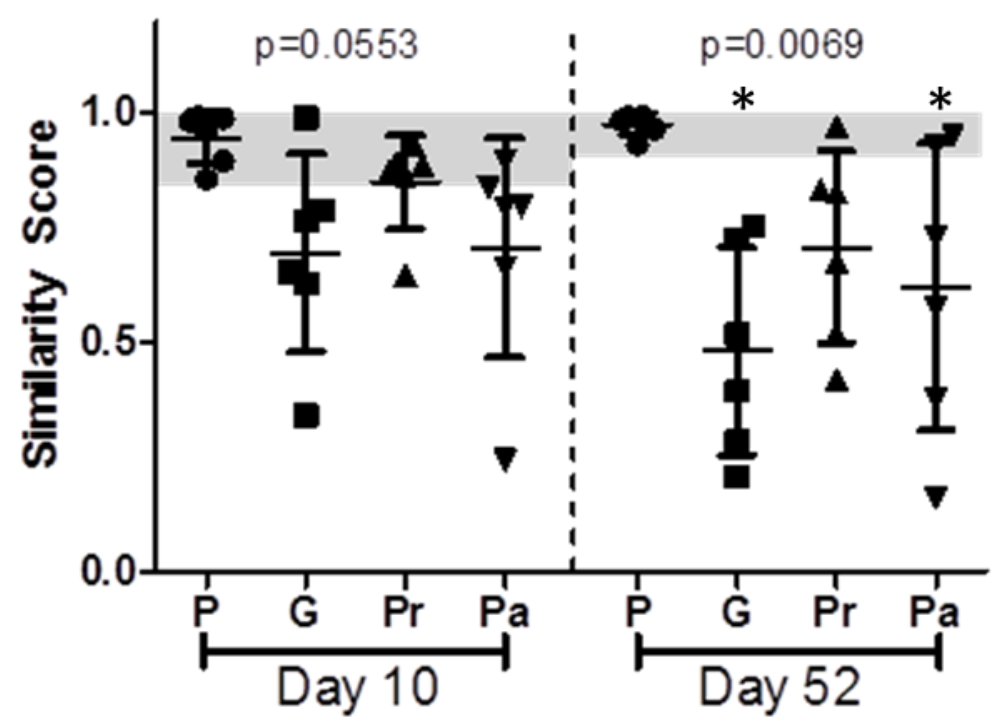

Figure 5.8. Thermogram similarity scores for sera from rats chronically treated with PBS or GRFT. Similarity scores were calculated for each thermogram generated from serum from PBS [P], GRFT [G], Pre-immunized/GRFT-treated [Pr] and Passively-immunized/GRFT-treated [Pa] animals by statistical comparison of curves versus a time-matched, mean PBS-treated control curve. Mean similarity values for day 10 and 52 similarity scores were analyzed by oneway ANOVA with Bonferroni post-test. Bars represent mean plus standard deviation of six biological replicates. A p-value of $<0.05$ was deemed significant $\left({ }^{*}\right)$. 


\begin{tabular}{|c|c|c|c|c|c|c|c|c|c|}
\hline & $\mathrm{T}_{\mathrm{FM}}$ & $\begin{array}{l}\text { PSV. } \\
\text { NEUT. }\end{array}$ & $\begin{array}{l}\text { ANTI- } \\
\text { GRFT } \\
\text { TITER }\end{array}$ & WIDTH & $\mathrm{T}_{\text {MAX }}$ & GLOB & T.P & $\begin{array}{c}\text { MAX } \\
\text { PEAK }\end{array}$ & AREA \\
\hline ALB. & 0.02 & -0.06 & -0.50 & 0.13 & 0.34 & -0.07 & 0.85 & -0.27 & -0.13 \\
\hline AREA & -0.19 & -0.02 & 0.21 & 0.44 & 0.04 & -0.21 & -0.14 & 0.92 & \\
\hline $\begin{array}{l}\text { MAX } \\
\text { PEAK }\end{array}$ & 0.07 & -0.14 & 0.23 & 0.12 & 0.03 & -0.08 & -0.19 & & \\
\hline $\begin{array}{l}\text { TOTAL } \\
\text { PROT }\end{array}$ & 0.28 & 0.04 & -0.19 & -0.08 & 0.35 & 0.49 & & & \\
\hline GLOB. & 0.50 & 0.12 & 0.45 & -0.46 & 0.20 & & & & \\
\hline$T_{\text {MAX }}$ & 0.52 & -0.32 & -0.05 & -0.10 & & & & & \\
\hline WIDTH & -0.56 & -0.02 & -0.05 & & & & & & \\
\hline $\begin{array}{l}\text { ANTI- } \\
\text { GRFT } \\
\text { TITER }\end{array}$ & -0.01 & 0.41 & & & & & & & \\
\hline $\begin{array}{l}\text { PSV. } \\
\text { NEUT. }\end{array}$ & -0.54 & & & & & & & & \\
\hline
\end{tabular}

Table 5.4. Pearson Correlations of rat serum thermogram parameters and rat serum characteristics. Day 52 thermogram parameters of Area, Max Peak, $T_{\max }$, $\mathrm{T}_{\mathrm{fm}}$, and Width were compared with day 55 serum characteristics of Albumin, Globulin, Total Protein (T.P), Anti-GRFT antibody titers, and pseudovirus neutralization (Psv. Neut.) from the same animal. Pearson Correlation and pvalues were generated. A correlation having a $p$-value of $p<0.05$ was considered significant. 


\section{CHAPTER 6}

\section{SUMMARY OF GRFT RESEARCH AND IMPLICATIONS FOR FUTURE RESEARCH}

Throughout this work, we have analyzed GRFT's biochemical interactions and physiological profile in furtherance of our overarching hypothesis that GRFT is a good candidate for antiviral prophylactic and therapeutics. A number of hypotheses were explored. First, we hypothesized that GRFT would be orally bioavailable after oral dosing. To test this hypothesis, we performed pharmacokinetic testing as well as chronic oral dosing of GRFT. Next, we hypothesized that chronic treatment of GRFT would be systemically tolerable. To test this hypothesis, we conducted a 51 day treatment regimen with GRFT in the presence and absence of a pre-existing immune response. Finally, given data from prior experiments, we hypothesized that biochemical interactions of GRFT may include specific binding interactions of importance. Further we hypothesized that while GRFT treatment did not cause significant toxicities in prior studies, perturbations in endogenous serum binding proteins may occur. To 
test these hypotheses, we conducted DSF, affinity purification of serum proteins, as well as DSC analysis of serum from chronically treated rats. From the data presented in this work, a number of conclusions can be drawn, which taken as a whole, support the further study of GRFT as a systemic antiviral therapeutic.

GRFT persisted in the circulation similarly upon dosed subcutaneously and intravenously.

Pharmacokinetic studies of GRFT's elimination profile revealed a number of insights into GRFT's serum parameters. First, GRFT does not appear to be extensively distributed in body water following intravenous administration. Second, GRFT displays a multi-phasic half-life, with half-life increasing after a very rapid elimination phase. GRFT concentrations 8 hours after either subcutaneous or intravenous administration were comparable, suggesting that in non-emergency settings, subcutaneous dosing may be a viable selfadministration method. Regardless, physiologically relevant concentrations GRFT persisted for up to 96 hours in serum whether dosed intravenously or subcutaneously.

Active GRFT is rapidly excreted by the kidneys, though proteolytic degradation may be occurring.

Studies into the method of elimination displayed a strong excretion of active GRFT concentrations immediately following intravenous dosing. However, over a 24 hour period, this fraction of GRFT excreted only accounted for approximately $1-2 \%$ of all GRFT dosed into the system. Based upon studies of 
others detailing GRFT's generally strong proteolytic resistance, yet susceptibility to specific proteases, it is hypothesized that rapid protein degradation of free GRFT may be occurring.

GRFT is not orally bioavailable at a detectable extent.

Both single oral dosing and 10-day chronic oral dosing revealed GRFT is not orally bioavailable to a detectable level. A single oral dose of GRFT, tracked at 8 time points after oral administration failed to yield detectable concentrations of GRFT in either serum or urine. Further, a 10-day chronic oral dosing study was conducted, to account for the possibility of an accumulation effect. All animals in that study, in which animals were orally administered by gavage with GRFT at two concentrations, failed to display detectable GRFT concentrations in serum at either day 5 or 3 hours after dosing on day 10 . These results would confirm that orally dosed GRFT is incapable of achieving potentially therapeutic concentrations in serum.

GRFT is a potential orally dosed rectal microbicide.

Despite studies showing GRFT is not orally bioavailable in rats, active GRFT concentrations were found in fecal material of rats orally dosed with GRFT. These concentrations were dependent upon amount of GRFT orally dosed. These GRFT concentrations, however, were only a very small fraction of GRFT dosed. Nonetheless, fecal extracts prepared from fecal pellets displayed strong neutralization activity in HIV-env pseudovirus neutralization assays. Accordingly, it appears that a fraction of active GRFT can pass through the 
digestive system intact. Given the site of many HIV infections are in the rectal mucosa, oral dosing of GRFT may be a method of delivering active, potentially protective concentrations of the protein to the rectal mucosa.

GRFT is tolerable in sub chronic dosing.

Subcutaneous dosing of GRFT every 3 days was tolerable over a 51 day period. No changes were observed in CBC values, body weights, or weight gain. Further, pathologist review of organ slides did not reveal any treatment related pathologies. There were alterations in kidney weights with pre-immunized animals. Further, alterations in some serum chemistries and cytokine levels were observed. However, varying form of immune response (pre or passive immunization) did not affect GRFT concentrations in serum or HIV-env pseudovirus neutralization activity. Accordingly, GRFT would appear to be systemically tolerable for intermediate term usage as an antiviral prophylactic or therapeutic.

GRFT treatment can induce changes in serum proteome with chronic dosing.

Calorimetric analysis of serum protein interactions displayed changes over time associated with GRFT treatment. At day 10, visual inspection of serum thermograms revealed fairly analogous mean thermograms for all treatment groups. Furthermore, no changes were observed in any curve parameter other than First moment temperature $\left(\mathrm{T}_{\mathrm{fm}}\right)$. In that group, pre-and passively immunized animals displayed a mean $\mathrm{T}_{\mathrm{fm}}$ lower than PBS or GRFT treated groups. However, by day 52, there were significant alterations in treatment group 
thermograms in comparison to PBS-treated controls. Many parameters analyzed revealed differences among groups, including passively-immunized and GRFT treated groups. However, it appeared that pre-immunization was able to blunt alterations in parameters. Pre-immunized groups were not generally statistically different in their curve parameters versus PBS-treated controls. This would seem to indicate a protective effect of an immune response associated with GRFT treatment.

GRFT binding, in and of itself, does not shift serum thermograms.

While significant shifts in rat serum thermogram parameters were observed following chronic treatment with GRFT, those shifts do not appear to be attributed to the direct binding activity of GRFT. Serum spiked with varying concentrations of GRFT and allowed to react for approximately 1 hour did not display alterations in serum thermograms. With spiking concentrations greatly exceeding those concentrations observed in serum following system administration, it was believed that any characteristic binding changes would be observed by saturation with GRFT. Given the lack of thermogram alteration in spiked samples, it would appear that alterations in serum thermograms following chronic GRFT treatment can be attributed to an immune response or alteration of concentrations of low molecular weight proteins which bind to the more abundant serum proteins. 
GRFT is capable of specific binding to proteins which do not possess oligomannose glycans

Given GRFT's long serum persistence observed following single and chronic dosing, DSC and AP studies were conducted to detail the potential interaction of GRFT with a number of ligands. Fluorometry results confirmed specific binding activity of GRFT to a number of saccharide ligands including glucose, mannose, maltose, mannan, and $\mathrm{N}$-acetylglucosamine. These interactions have been observed in other previous studies. Further, affinity purification of human serum proteins with both FLAG-GRFT and FLAG-GRFT'lecfusion proteins revealed a number of potentially relevant biological interactions. Among these, interactions with complement and apoplipoproteins were the most numerous in FLAG-GRFT purified groups. These data would confirm specific binding interactions beyond $\mathrm{N}$-linked oligomannose binding activity. However, the effects of these interactions will require additional study. 


\section{Summary}

In summary, GRFT has displayed persistent anti-viral activity in serum, even in the presence of an immune response, following systemic administration. Further, physiologically relevant concentrations can remain in serum days following subcutaneous administration. While long-term dosing may induce biochemical changes and may have an immunosuppressive effect that will require additional study, it would appear that subchronic dosing of GRFT is tolerable. Additionally, while lacking oral bioavailability in rats, oral administrations of GRFT may have utility. A small fraction of orally dosed GRFT can pass into the rectal mucosa via fecal material. This transit of active GRFT may eventually allow for the usage of GRFT as an orally dosed rectal microbicide. 


\section{RECOMMENDATIONS FOR FURTHER STUDY}

While GRFT is still a strong candidate for anti-viral prophylaxis and therapy, a number of questions have been raised by these studies. It is recommended that further research be conducted to address these questions.

What is the primary mode of GRFT degradation?

The studies in this work have consistently shown that GRFT can persist in serum for a long period of time. However, the vast majority of GRFT dosed disappears within 24 hours of dosing. Mass Balance experiments revealed only a small fraction of GRFT was excreted through the kidneys after an initial burst of excretion. Thus, it would appear that GRFT is degraded in some other manner. Therefore, radiolabeling experiments may be necessary to follow GRFT's journey through systemic circulation. The radiolabeling could also be useful in tracking the extent of GRFT's distribution as well as persistence in specific organ groups.

\section{What impact does GRFT have upon the kidneys?}

Sub-chronic dosing of GRFT did display changes in kidney size when an immune response was induced. However, at the day 55 of the study, pathological differences were not observable between kidneys. A long term administration of GRFT in the presence of an immune response may be required in order to clearly delineate alterations in kidney structure and function. It is also recommended that blood nitrogen urea, creatinine clearance, and total glomerular filtration rate be tracked over the course of treatment. These may provide valuable insights into GRFT's effect on the kidneys. 
Does GRFT cause immunosuppression or immunotoxicity?

Sub-chronic dosing of GRFT in rats was observed to lower some inflammatory cytokine levels in comparison to adjuvant pre-immunized animals and PBS-treated controls. Further, affinity purification of human serum revealed complement factors were recovered by FLAG-GRFT and FLAG-GRFT ${ }^{\text {lec- }}$. This suggests that GRFT administration may have some immunosuppressive or immunotoxic effect that could impair an immune response to invading pathogens. Accordingly, it is suggested that GRFT complement binding be confirmed by binding ELISA as well as the nature of GRFT's mode of action to rule out a functional depletion of complement. Therefore, additional in vivo and in vitro tests will need to be carried to out determine whether GRFT impairs humoral response, cellular responses of T-cells, natural killer cells, macrophages, or granulocytes. Further, testing of host resistance to infection (bacterial, viral, and tumor) will be necessary, with a concomitant observation for illness.

Can concentrations of active GRFT found in fecal matter be increased?

Oral dosing studies discovered that active GRFT is excreted in fecal matter. Given that HIV infection often entails exposure of fluids to rectal mucosa, GRFT's presence in fecal material passing through the rectum may enable its usage as a rectal microbicide. It is possible that GRFT presence in fecal material will be able to seed rectal mucosa with potentially protective concentrations of GRFT. However, mass balance studies detailed only limited passage of active GRFT. Accordingly studies into methods of increasing active concentrations of 
GRFT may further highlight GRFT's potential as an orally dosed rectal microbicide. These could include dosage of GRFT concurrent with antacids, protease inhibitors, or in nanoparticle suspension or controlled release encapsulation. Further, experiments with radiolabeled GRFT may be useful to determine whether degraded GRFT is systemically absorbed.

Does GRFT treatment affect the gut microbiome?

While GRFT may be a promising orally dosed rectal microbicide, its impact on the gut microbiome will need to be assessed. The gut microbiome can be crucial in health, as well as immune system function and regulation [Citations needed]. Agents which alter the endogenous balance can result in loss of beneficial bacteria or a blooming of harmful bacteria. Given that some bacteria, such as E. coli use mannose for adhesion [243, 244], it is possible that GRFT presence could compete with endogenous flora for mannose or other adhesion molecules. Accordingly, a tracking of the speed and nature of gut microbiome changes over long term oral administration of GRFT will be necessary to eliminate the potential that oral GRFT treatment could result in negative health consequences.

Determine the nature of GRFT's interaction with apolipoproteins and complement

GRFT's binding to human serum proteins has been documented in this study. Particularly relevant were binding interactions with complement and apolipoproteins. In particular, the binding with complement could have physiological relevance. Complement deficiencies have been associated with 
autoimmune disease. Thus, a functional depletion of complement through binding interactions with GRFT in a chronic administration model could potentially exacerbate those disease associations. Accordingly, determining the nature of those interactions will be necessary. Experiments entailing surface plasmon resonance assays, isothermal titration calorimetry, and biotin tag affinity switching using a photosensitive cross-linker may yield important information regarding specific binding interactions with GRFT.

How does chronic administration of GRFT affect serum thermograms?

Spiking of rat serum with GRFT did not alter thermogram parameters. However, chronic administration of GRFT resulted in significant changes in comparison to PBS treated controls. However, the exact causative nature of these changes are unknown. DSC can yield information as to characteristic changes that occur, but is limited to describe the exact nature of the protein interactions causing the change. Accordingly, quantitative studies will be necessary into the abundance of lower molecular weight proteins present in altered samples. It is recommended that these chronic dosing studies be repeated solely for the purpose of comparing the alterations in lower molecular weight proteins. Cytokine panels may yield rough concentration changes in GRFT treated groups. Further, MS analysis may be useful in identifying changes in serum components - however, Ig and Serum Albumin depletion may be required before sample processing to remove signal masking by the more abundant proteins. 
Whether GRFT can be utilized in long term therapy (>6 month)

Studies to date have yielded positive results in examining GRFT's potential as a systemic therapeutic for viruses possess $\mathrm{N}$-linked, oligomannose glycans. However, given the changes observed in a 55-day treatment regimen, longer-term studies will be necessary to determine the true extent of changes induced. Parameters such as kidney weight, blood urea nitrogen, spleen weight, and anti-GRFT antibody activity should be monitored to determine whether GRFT will be suitable for long-term usage. 


\section{REFERENCES}

1. www.UNAIDS.org, retrieved 5/1/2014.

2. Guha, D. and V. Ayyavoo, Innate Immune Evasion Strategies by Human Immunodeficiency Virus Type 1. ISRN AIDS, 2013. 2013: p. 954806.

3. Jin, R., et al., Japanese encephalitis virus activates autophagy as a viral immune evasion strategy. PLoS One, 2013. 8(1): p. e52909.

4. Basler, C.F., A novel mechanism of immune evasion mediated by Ebola virus soluble glycoprotein. Expert Rev Anti Infect Ther, 2013. 11(5): p. 475-8.

5. Mohan, G.S., et al., Antigenic subversion: a novel mechanism of host immune evasion by Ebola virus. PLoS Pathog, 2012. 8(12): p. e1003065.

6. Draenert, R., J. Frater, and J.G. Prado, Virus immune evasion: new mechanism and implications in disease outcome. Adv Virol, 2012. 2012: p. 490549.

7. Lewis, M.J., et al., Immune selection in vitro reveals human immunodeficiency virus type 1 Nef sequence motifs important for its immune evasion function in vivo. J Virol, 2012. 86(13): p. 7126-35.

8. Price, D.A., et al., The influence of antigenic variation on cytotoxic $T$ lymphocyte responses in HIV-1 infection. J Mol Med (Berl), 1998. 76(10): p. 699-708.

9. Wolfs, T.F., et al., Naturally occurring mutations within HIV-1 V3 genomic $R N A$ lead to antigenic variation dependent on a single amino acid substitution. Virology, 1991. 185(1): p. 195-205.

10. Martinelli, E., et al., HIV-1 gp120 inhibits TLR9-mediated activation and IFN-\{alpha\} secretion in plasmacytoid dendritic cells. Proc Natl Acad Sci U S A, 2007. 104(9): p. 3396-401.

11. Bennasser, Y., et al., RNA interference and HIV-1: hits and misses. Curr Opin HIV AIDS, 2006. 1(3): p. 208-11.

12. Bennasser, Y., et al., Evidence that HIV-1 encodes an siRNA and a suppressor of RNA silencing. Immunity, 2005. 22(5): p. 607-19.

13. Bonomelli, C., et al., The glycan shield of HIV is predominantly oligomannose independently of production system or viral clade. PLoS One, 2011. 6(8): p. e23521.

14. Balzarini, J., Targeting the glycans of glycoproteins: a novel paradigm for antiviral therapy. Nat Rev Microbiol, 2007. 5(8): p. 583-97. 
15. Bertaux, C., et al., Entry of hepatitis $C$ virus and human immunodeficiency virus is selectively inhibited by carbohydrate-binding agents but not by polyanions. Virology, 2007. 366(1): p. 40-50.

16. Alexandre, K.B., et al., Mannose-rich glycosylation patterns on HIV-1 subtype $C$ gp120 and sensitivity to the lectins, Griffithsin, Cyanovirin-N and Scytovirin. Virology, 2010. 402(1): p. 187-96.

17. Helle, F., et al., Role of $N$-linked glycans in the functions of hepatitis $C$ virus envelope proteins incorporated into infectious virions. J Virol, 2010. 84(22): p. 11905-15.

18. Mascola, J.R. and D.C. Montefiori, HIV-1: nature's master of disguise. Nat Med, 2003. 9(4): p. 393-4.

19. Shan, M., et al., HIV-1 gp120 mannoses induce immunosuppressive responses from dendritic cells. PLoS Pathog, 2007. 3(11): p. e169.

20. Banerjee, K., et al., Enzymatic removal of mannose moieties can increase the immune response to HIV-1 gp120 in vivo. Virology, 2009. 389(1-2): p. 108-21.

21. Balzarini, J., Targeting the glycans of gp120: a novel approach aimed at the Achilles heel of HIV. Lancet Infect Dis, 2005. 5(11): p. 726-31.

22. Balzarini, J., Inhibition of HIV entry by carbohydrate-binding proteins. Antiviral Res, 2006. 71(2-3): p. 237-47.

23. O'Keefe, B.R., et al., Broad-spectrum in vitro activity and in vivo efficacy of the antiviral protein griffithsin against emerging viruses of the family Coronaviridae. J Virol, 2010. 84(5): p. 2511-21.

24. Francois, K.O. and J. Balzarini, Potential of carbohydrate-binding agents as therapeutics against enveloped viruses. Med Res Rev, 2010.

25. Witvrouw, M., et al., Resistance of human immunodeficiency virus type 1 to the high-mannose binding agents cyanovirin $N$ and concanavalin $A$. J Virol, 2005. 79(12): p. 7777-84.

26. Zappe, H., M.E. Snell, and M.J. Bossard, PEGylation of cyanovirin-N, an entry inhibitor of HIV. Adv Drug Deliv Rev, 2008. 60(1): p. 79-87.

27. Tanaka, H., et al., Mechanism by which the lectin actinohivin blocks HIV infection of target cells. Proc Natl Acad Sci U S A, 2009. 106(37): p. 15633-8.

28. Smee, D.F., et al., Treatment of influenza $A$ (H1N1) virus infections in mice and ferrets with cyanovirin-N. Antiviral Res, 2008. 80(3): p. 266-71.

29. Michelow, I.C., et al., High-dose mannose-binding lectin therapy for Ebola virus infection. J Infect Dis, 2011. 203(2): p. 175-9.

30. Takebe, Y., et al., Antiviral Lectins from Red and Blue-Green Algae Show Potent In Vitro and In Vivo Activity against Hepatitis C Virus. PLoS One, 2013. 8(5): p. e64449.

31. Meuleman, P., et al., Griffithsin has antiviral activity against hepatitis $C$ virus. Antimicrob Agents Chemother, 2011. 55(11): p. 5159-67.

32. Mori, T., et al., Isolation and characterization of griffithsin, a novel HIVinactivating protein, from the red alga Griffithsia sp. J Biol Chem, 2005. 280(10): p. 9345-53. 
33. Emau, P., et al., Griffithsin, a potent HIV entry inhibitor, is an excellent candidate for anti-HIV microbicide. J Med Primatol, 2007. 36(4-5): p. 24453.

34. Barton, C., et al., Activity of and effect of subcutaneous treatment with the broad-spectrum antiviral lectin griffithsin in two laboratory rodent models. Antimicrob Agents Chemother, 2014. 58(1): p. 120-7.

35. Kouokam, J.C., et al., Investigation of griffithsin's interactions with human cells confirms its outstanding safety and efficacy profile as a microbicide candidate. PLoS One, 2011. 6(8): p. e22635.

36. Nixon, B., et al., Griffithsin protects mice from genital herpes by preventing cell-to-cell spread. J Virol, 2013. 87(11): p. 6257-69.

37. Huskens, D. and D. Schols, Algal lectins as potential HIV microbicide candidates. Mar Drugs, 2012. 10(7): p. 1476-97.

38. Essentials of Glycobiology, 2nd Edition

39. Bosques, C.J., et al., Effects of glycosylation on peptide conformation: a synergistic experimental and computational study. J Am Chem Soc, 2004. 126(27): p. 8421-5.

40. Vigerust, D.J. and V.L. Shepherd, Virus glycosylation: role in virulence and immune interactions. Trends Microbiol, 2007. 15(5): p. 211-8.

41. Fundamentals of Biochemistry, Third Edition, Voet \& Pratt

42. Molecular Biology of the Cell, Fifth Edition, Alberts \& Walter

43. Green, R.S., et al., Mammalian N-glycan branching protects against innate immune self-recognition and inflammation in autoimmune disease pathogenesis. Immunity, 2007. 27(2): p. 308-20.

44. Wormald, M.R. and R.A. Dwek, Glycoproteins: glycan presentation and protein-fold stability. Structure, 1999. 7(7): p. R155-60.

45. Sperandio, M., C.A. Gleissner, and K. Ley, Glycosylation in immune cell trafficking. Immunol Rev, 2009. 230(1): p. 97-113.

46. Anthony, R.M., et al., Recapitulation of IVIG anti-inflammatory activity with a recombinant lgG Fc. Science, 2008. 320(5874): p. 373-6.

47. Nimmerjahn, F. and J.V. Ravetch, Anti-inflammatory actions of intravenous immunoglobulin. Annu Rev Immunol, 2008. 26: p. 513-33.

48. Su, D., H. Zhao, and H. Xia, Glycosylation-modified erythropoietin with improved half-life and biological activity. Int J Hematol, 2010. 91(2): p. 238-44.

49. Kanda, Y., et al., Comparison of biological activity among nonfucosylated therapeutic IgG1 antibodies with three different N-linked FC oligosaccharides: the high-mannose, hybrid, and complex types. Glycobiology, 2007. 17(1): p. 104-18.

50. Goffard, A. and J. Dubuisson, Glycosylation of hepatitis $C$ virus envelope proteins. Biochimie, 2003. 85(3-4): p. 295-301.

51. Helle, F., G. Duverlie, and J. Dubuisson, The hepatitis $C$ virus glycan shield and evasion of the humoral immune response. Viruses, 2011. 3(10): p. 1909-32. 
52. Liu, M., et al., Deletion of $\mathrm{N}$-glycosylation sites of hepatitis $C$ virus envelope protein $E 1$ enhances specific cellular and humoral immune responses. Vaccine, 2007. 25(36): p. 6572-80.

53. Ward, A.C., Virulence of influenza A virus for mouse lung. Virus Genes, 1997. 14(3): p. 187-94.

54. Ritchie, G., et al., Identification of N-linked carbohydrates from severe acute respiratory syndrome (SARS) spike glycoprotein. Virology, 2010. 399(2): p. 257-69.

55. Han, D.P., M. Lohani, and M.W. Cho, Specific asparagine-linked glycosylation sites are critical for DC-SIGN- and L-SIGN-mediated severe acute respiratory syndrome coronavirus entry. J Virol, 2007. 81(21): p. 12029-39.

56. Feldmann, $\mathrm{H}$., et al., Characterization of filoviruses based on differences in structure and antigenicity of the virion glycoprotein. Virology, 1994. 199(2): p. 469-73.

57. Dowling, W., et al., Influences of glycosylation on antigenicity, immunogenicity, and protective efficacy of ebola virus GP DNA vaccines. J Virol, 2007. 81(4): p. 1821-37.

58. Douek, D.C., M. Roederer, and R.A. Koup, Emerging concepts in the immunopathogenesis of AIDS. Annu Rev Med, 2009. 60: p. 471-84.

59. Swanson, C.M. and M.H. Malim, SnapShot: HIV-1 proteins. Cell, 2008. 133(4): p. 742, 742 e1.

60. Doores, K.J., et al., Envelope glycans of immunodeficiency virions are almost entirely oligomannose antigens. Proc Natl Acad Sci U S A, 2010. 107(31): p. 13800-5.

61. Li, Q., et al., Peak SIV replication in resting memory CD4+ T cells depletes gut lamina propria CD4+ T cells. Nature, 2005. 434(7037): p. 1148-52.

62. Zhang, Z.Q., et al., Roles of substrate availability and infection of resting and activated $C D 4+T$ cells in transmission and acute simian immunodeficiency virus infection. Proc Natl Acad Sci U S A, 2004. 101(15): p. 5640-5.

63. Zhang, Z., et al., Sexual transmission and propagation of SIV and HIV in resting and activated CD4+ T cells. Science, 1999. 286(5443): p. 1353-7.

64. Nawaz, F., et al., The genotype of early-transmitting HIV gp120s promotes alphabeta-reactivity, revealing alphabetaCD4+ $T$ cells as key targets in mucosal transmission. PLoS Pathog, 2011. 7(2): p. e1001301.

65. Haase, A.T., Perils at mucosal front lines for HIV and SIV and their hosts. Nat Rev Immunol, 2005. 5(10): p. 783-92.

66. Shacklett, B.L., et al., Mucosal T-cell responses to HIV: responding at the front lines. J Intern Med, 2009. 265(1): p. 58-66.

67. www.cdc.gov/hiv/statistics; retrieved $5 / 1 / 14$

68. O'Connor, D.M., A tissue basis for colposcopic findings. Obstet Gynecol Clin North Am, 2008. 35(4): p. 565-82; viii.

69. Pudney, J., A.J. Quayle, and D.J. Anderson, Immunological microenvironments in the human vagina and cervix: mediators of cellular 
immunity are concentrated in the cervical transformation zone. Biol Reprod, 2005. 73(6): p. 1253-63.

70. Miller, C.J. and R.J. Shattock, Target cells in vaginal HIV transmission. Microbes Infect, 2003. 5(1): p. 59-67.

71. Grivel, J.C., R.J. Shattock, and L.B. Margolis, Selective transmission of R5 HIV-1 variants: where is the gatekeeper? J Transl Med, 2011. 9 Suppl 1: p. S6.

72. Chenine, A.L., et al., Relative transmissibility of an $R 5$ clade $C$ simianhuman immunodeficiency virus across different mucosae in macaques parallels the relative risks of sexual HIV-1 transmission in humans via different routes. J Infect Dis, 2010. 201(8): p. 1155-63.

73. Olsson, J., et al., Human immunodeficiency virus type 1 infection is associated with significant mucosal inflammation characterized by increased expression of CCR5, CXCR4, and beta-chemokines. J Infect Dis, 2000. 182(6): p. 1625-35.

74. Nazli, A., et al., Exposure to HIV-1 directly impairs mucosal epithelial barrier integrity allowing microbial translocation. PLoS Pathog, 2010. 6(4): p. e1000852.

75. Poles, M.A., et al., A preponderance of CCR5(+) CXCR4(+) mononuclear cells enhances gastrointestinal mucosal susceptibility to human immunodeficiency virus type 1 infection. J Virol, 2001. 75(18): p. 8390-9.

76. Bomsel, M., Transcytosis of infectious human immunodeficiency virus across a tight human epithelial cell line barrier. Nat Med, 1997. 3(1): p. 427.

77. Shen, R., et al., Dendritic cells transmit HIV-1 through human small intestinal mucosa. J Leukoc Biol, 2010. 87(4): p. 663-70.

78. McDougal, J.S., D.R. Klatzmann, and P.J. Maddon, CD4-gp120 interactions. Curr Opin Immunol, 1991. 3(4): p. 552-8.

79. Dalgleish, A.G., et al., The CD4 (T4) antigen is an essential component of the receptor for the AIDS retrovirus. Nature, 1984. 312(5996): p. 763-7.

80. Sattentau, Q.J. and R.A. Weiss, The CD4 antigen: physiological ligand and HIV receptor. Cell, 1988. 52(5): p. 631-3.

81. Myszka, D.G., et al., Energetics of the HIV gp120-CD4 binding reaction. Proc Natl Acad Sci U S A, 2000. 97(16): p. 9026-31.

82. Cormier, E.G. and T. Dragic, The crown and stem of the V3 loop play distinct roles in human immunodeficiency virus type 1 envelope glycoprotein interactions with the CCR5 coreceptor. J Virol, 2002. 76(17): p. 8953-7.

83. Suphaphiphat, P., M. Essex, and T.H. Lee, Mutations in the V3 stem versus the V 3 crown and $C 4$ region have different effects on the binding and fusion steps of human immunodeficiency virus type $1 \mathrm{gp} 120$ interaction with the CCR5 coreceptor. Virology, 2007. 360(1): p. 182-90.

84. Briz, V., E. Poveda, and V. Soriano, HIV entry inhibitors: mechanisms of action and resistance pathways. J Antimicrob Chemother, 2006. 57(4): p. 619-27. 
85. Weiss, C.D., HIV-1 gp41: mediator of fusion and target for inhibition. AIDS Rev, 2003. 5(4): p. 214-21.

86. Poveda, E., V. Briz, and V. Soriano, Enfuvirtide, the first fusion inhibitor to treat HIV infection. AIDS Rev, 2005. 7(3): p. 139-47.

87. Life expectancy of individuals on combination antiretroviral therapy in high-income countries: a collaborative analysis of 14 cohort studies. Lancet, 2008. 372(9635): p. 293-9.

88. Li, T.S., et al., Long-lasting recovery in CD4 T-cell function and viral-load reduction after highly active antiretroviral therapy in advanced HIV-1 disease. Lancet, 1998. 351(9117): p. 1682-6.

89. Wilson, E.M. and I. Sereti, Immune restoration after antiretroviral therapy: the pitfalls of hasty or incomplete repairs. Immunol Rev, 2013. 254(1): p. 343-54.

90. Corbeau, P. and J. Reynes, Immune reconstitution under antiretroviral therapy: the new challenge in HIV-1 infection. Blood, 2011. 117(21): $\mathrm{p}$. 5582-90.

91. Pitrak, D.L., et al., Discordant CD4 T lymphocyte responses to antiretroviral therapy for HIV infection are associated with ex-vivo rates of apoptosis. AIDS, 2001. 15(10): p. 1317-9.

92. Gallant, J.E., S.H. Mehta, and J. Sugarman, Universal antiretroviral therapy for HIV infection: should US treatment guidelines be applied to resource-limited settings? Clin Infect Dis, 2013. 57(6): p. 884-7.

93. Richardson, E.T., P.M. Grant, and A.R. Zolopa, Evolution of HIV treatment guidelines in high- and low-income countries: converging recommendations. Antiviral Res, 2014. 103: p. 88-93.

94. Rong, L. and A.S. Perelson, Asymmetric division of activated latently infected cells may explain the decay kinetics of the HIV-1 latent reservoir and intermittent viral blips. Math Biosci, 2009. 217(1): p. 77-87.

95. Kaufmann, G.R., et al., CD4 T-lymphocyte recovery in individuals with advanced HIV-1 infection receiving potent antiretroviral therapy for 4 years: the Swiss HIV Cohort Study. Arch Intern Med, 2003. 163(18): p. 2187-95.

96. Geng, E.H. and S.G. Deeks, CD4+ T cell recovery with antiretroviral therapy: more than the sum of the parts. Clin Infect Dis, 2009. 48(3): $p$. 362-4.

97. Le, T., et al., Enhanced CD4+ T-cell recovery with earlier HIV-1 antiretroviral therapy. N Engl J Med, 2013. 368(3): p. 218-30.

98. Alexaki, A., Y. Liu, and B. Wigdahl, Cellular reservoirs of HIV-1 and their role in viral persistence. Curr HIV Res, 2008. 6(5): p. 388-400.

99. Belmonte, L., et al., Reservoirs of HIV replication after successful combined antiretroviral treatment. Curr Med Chem, 2003. 10(4): p. 30312.

100. McNamara, L.A. and K.L. Collins, Hematopoietic stem/precursor cells as HIV reservoirs. Curr Opin HIV AIDS, 2011. 6(1): p. 43-8. 
101. Shen, L. and R.F. Siliciano, Viral reservoirs, residual viremia, and the potential of highly active antiretroviral therapy to eradicate HIV infection. $J$ Allergy Clin Immunol, 2008. 122(1): p. 22-8.

102. Mavigner, M., et al., HIV-1 residual viremia correlates with persistent T-cell activation in poor immunological responders to combination antiretroviral therapy. PLoS One, 2009. 4(10): p. e7658.

103. van Sighem, A., et al., Immunologic, virologic, and clinical consequences of episodes of transient viremia during suppressive combination antiretroviral therapy. J Acquir Immune Defic Syndr, 2008. 48(1): p. 104-8.

104. Girard, P.M., et al., Can we stop CD4+ testing in patients with HIV-1 RNA suppression on antiretroviral treatment? AIDS, 2013. 27(17): p. 2759-63.

105. Baeten, J.M., et al., Antiretroviral prophylaxis for HIV prevention in heterosexual men and women. N Engl J Med, 2012. 367(5): p. 399-410.

106. Centers for Disease, $C$. and Prevention, Interim guidance for clinicians considering the use of preexposure prophylaxis for the prevention of HIV infection in heterosexually active adults. MMWR Morb Mortal Wkly Rep, 2012. 61(31): p. 586-9.

107. www.cdc.gov/hiv/pdf/PrEPguidelines2014.pdf; retrieved 5/1/14

108. Celum, C.L., HIV preexposure prophylaxis: new data and potential use. Top Antivir Med, 2011. 19(5): p. 181-5.

109. Coutinho, B. and R. Prasad, Emtricitabine/tenofovir (Truvada) for HIV prophylaxis. Am Fam Physician, 2013. 88(8): p. 535-40.

110. Kidney damage reported in some tenofovir users. TreatmentUpdate, 2003. 15(2): p. 6-7.

111. Perazella, M.A., Tenofovir-induced kidney disease: an acquired renal tubular mitochondriopathy. Kidney Int, 2010. 78(11): p. 1060-3.

112. Rodriguez-Novoa, S., et al., Impairment in kidney tubular function in patients receiving tenofovir is associated with higher tenofovir plasma concentrations. AIDS, 2010. 24(7): p. 1064-6.

113. www.who.int/hiv/pub/mtct/programmatic update tasp/en/; retrieved $5 / 1 / 14$.

114. Rodger A et. al. HIV Transmission Risk through Condomless Sex if HIV+ Partner on Suppressive ART. PARTNER study. 21st Conference on Retroviruses and Opportunistic Infections, Boston, Abstract 153LB, 2014.

115. Cohen, M.S., M. McCauley, and T.R. Gamble, HIV treatment as prevention and HPTN 052. Curr Opin HIV AIDS, 2012. 7(2): p. 99-105.

116. Cohen, M.S., et al., Antiretroviral treatment of HIV-1 prevents transmission of HIV-1: where do we go from here? Lancet, 2013. 382(9903): p. 151524.

117. Post-exposure prophylaxis for HIV. Drug Ther Bull, 2011. 49(3): p. 30-3; quiz i.

118. Flexner, C., Post-exposure prophylaxis revisited: new CDC guidelines. Centers for Disease Control and Prevention. Hopkins HIV Rep, 1998. 10(1): p. 2-3.

119. Hardy, E.J., Testing and treatment after non-occupational exposures to STDs and HIV. Med Health R I, 2012. 95(8): p. 258-61. 
120. Young, T.N., et al., Antiretroviral post-exposure prophylaxis (PEP) for occupational HIV exposure. Cochrane Database Syst Rev, 2007(1): p. CD002835.

121. Watson, C., et al., The CCR5 receptor-based mechanism of action of 873140 , a potent allosteric noncompetitive HIV entry inhibitor. Mol Pharmacol, 2005. 67(4): p. 1268-82.

122. Burton, D.R., et al., A large array of human monoclonal antibodies to type 1 human immunodeficiency virus from combinatorial libraries of asymptomatic seropositive individuals. Proc Natl Acad Sci U S A, 1991. 88(22): p. 10134-7.

123. Trkola, A., et al., Human monoclonal antibody 2G12 defines a distinctive neutralization epitope on the gp120 glycoprotein of human immunodeficiency virus type 1. J Virol, 1996. 70(2): p. 1100-8.

124. Shingai, M., et al., Antibody-mediated immunotherapy of macaques chronically infected with SHIV suppresses viraemia. Nature, 2013. 503(7475): p. 277-80.

125. Barouch, D.H., et al., Therapeutic efficacy of potent neutralizing HIV-1specific monoclonal antibodies in SHIV-infected rhesus monkeys. Nature, 2013. 503(7475): p. 224-8.

126. Kuritzkes, D.R., HIV-1 entry inhibitors: an overview. Curr Opin HIV AIDS, 2009. 4(2): p. 82-7.

127. Dimitrov, A., Ibalizumab, a CD4-specific $m A b$ to inhibit HIV-1 infection. Curr Opin Investig Drugs, 2007. 8(8): p. 653-61.

128. Bruno, C.J. and J.M. Jacobson, Ibalizumab: an anti-CD4 monoclonal antibody for the treatment of HIV-1 infection. J Antimicrob Chemother, 2010. 65(9): p. 1839-41.

129. Song, R., et al., Epitope mapping of ibalizumab, a humanized anti-CD4 monoclonal antibody with anti-HIV-1 activity in infected patients. J Virol, 2010. 84(14): p. 6935-42.

130. Jacobson, J.M., et al., Safety, pharmacokinetics, and antiretroviral activity of multiple doses of ibalizumab (formerly TNX-355), an anti-CD4 monoclonal antibody, in human immunodeficiency virus type 1-infected adults. Antimicrob Agents Chemother, 2009. 53(2): p. 450-7.

131. Kuritzkes, D.R., et al., Antiretroviral activity of the anti-CD4 monoclonal antibody TNX-355 in patients infected with HIV type 1. J Infect Dis, 2004. 189(2): p. 286-91.

132. Fletcher, C.V., Enfuvirtide, a new drug for HIV infection. Lancet, 2003. 361(9369): p. 1577-8.

133. Jamjian, M.C. and I.R. McNicholl, Enfuvirtide: first fusion inhibitor for treatment of HIV infection. Am J Health Syst Pharm, 2004. 61(12): p. $1242-7$.

134. Kitchen, C.M., et al., Enfuvirtide antiretroviral therapy in HIV-1 infection. Ther Clin Risk Manag, 2008. 4(2): p. 433-9.

135. Robertson, D., US FDA approves new class of HIV therapeutics. Nat Biotechnol, 2003. 21(5): p. 470-1. 
136. Dorr, P., et al., Maraviroc (UK-427,857), a potent, orally bioavailable, and selective small-molecule inhibitor of chemokine receptor CCR5 with broad-spectrum anti-human immunodeficiency virus type 1 activity. Antimicrob Agents Chemother, 2005. 49(11): p. 4721-32.

137. Symons, J., et al., Maraviroc is able to inhibit dual-R5 viruses in a dual/mixed HIV-1-infected patient. J Antimicrob Chemother, 2011. 66(4): p. 890-5.

138. Soriano, V., et al., Optimal use of maraviroc in clinical practice. AIDS, 2008. 22(17): p. 2231-40.

139. Castonguay, L.A., et al., Binding of 2-aryl-4-(piperidin-1-yl)butanamines and 1,3,4-trisubstituted pyrrolidines to human CCR5: a molecular modeling-guided mutagenesis study of the binding pocket. Biochemistry, 2003. 42(6): p. 1544-50.

140. Chiba, $\mathrm{H}$., et al., Actinohivin, a novel anti-human immunodeficiency virus protein from an actinomycete, inhibits viral entry to cells by binding highmannose type sugar chains of gp120. Biochem Biophys Res Commun, 2004. 316(1): p. 203-10.

141. Bewley, C.A. and S. Otero-Quintero, The potent anti-HIV protein cyanovirin- $N$ contains two novel carbohydrate binding sites that selectively bind to Man(8) D1D3 and Man(9) with nanomolar affinity: implications for binding to the HIV envelope protein gp120. J Am Chem Soc, 2001. 123(17): p. 3892-902.

142. Swanson, M.D., et al., A lectin isolated from bananas is a potent inhibitor of HIV replication. J Biol Chem, 2010. 285(12): p. 8646-55.

143. Balzarini, J., et al., Profile of resistance of human immunodeficiency virus to mannose-specific plant lectins. J Virol, 2004. 78(19): p. 10617-27.

144. Kaczera, Z. and D. Stankiewicz, Influence of some phytohaemagglutinins (PHA) on the red blood cells of laboratory animals. Folia Biol (Krakow), 1969. 17(4): p. 321-31.

145. Larsson, E.L. and A. Coutinho, The role of mitogenic lectins in T-cell triggering. Nature, 1979. 280(5719): p. 239-41.

146. Vasconcelos, I.M. and J.T. Oliveira, Antinutritional properties of plant lectins. Toxicon, 2004. 44(4): p. 385-403.

147. Miyake, K., T. Tanaka, and P.L. McNeil, Lectin-based food poisoning: a new mechanism of protein toxicity. PLoS One, 2007. 2(8): p. e687.

148. Jonsson, T., et al., Agrarian diet and diseases of affluence--do evolutionary novel dietary lectins cause leptin resistance? BMC Endocr Disord, 2005. 5: p. 10.

149. Huskens, D., et al., Safety concerns for the potential use of cyanovirin-N as a microbicidal anti-HIV agent. Int J Biochem Cell Biol, 2008. 40(12): p. 2802-14.

150. Peumans, W.J., et al., Fruit-specific lectins from banana and plantain. Planta, 2000. 211(4): p. 546-54.

151. Koshte, V.L., et al., Isolation and characterization of BanLec-I, a mannoside-binding lectin from Musa paradisiac (banana). Biochem J, 1990. 272(3): p. 721-6. 
152. Essentials of Glycobiology, 2nd edition. Chapter 45. Varki, et. al. Cold Spring Harbor (NY): Cold Spring Harbor Laboratory Press; 2009.

153. Nishida, A., T. Kobayashi, and F. Ariyuki, Developmental toxicity of concanavalin $A$ in rats: association with restricted migration of neural crest cells. Food Chem Toxicol, 1996. 34(8): p. 701-8.

154. Nopanitaya, W., J. Hanker, and M. Tyan, Concanavalin A toxicity: histological studies. Proc Soc Exp Biol Med, 1976. 153(2): p. 213-9.

155. Tyan, M.L., In vivo toxicity of concanavalin A. Proc Soc Exp Biol Med, 1974. 146(4): p. 1163-5.

156. Cellular and Molecular immunology

157. Abud-Mendoza, C. and R. Gonzalez-Amaro, Rituximab in systemic lupus erythematosus. Lupus, 2010. 19(5): p. 658.

158. Kopp, M.V., Omalizumab: Anti-lgE Therapy in Allergy. Curr Allergy Asthma Rep, 2011. 11(2): p. 101-6.

159. Cox, L., et al., American Academy of Allergy, Asthma \& Immunology/American College of Allergy, Asthma and Immunology Joint Task Force Report on omalizumab-associated anaphylaxis. J Allergy Clin Immunol, 2007. 120(6): p. 1373-7.

160. Rituximab (Rituxan) for rheumatoid arthritis. Med Lett Drugs Ther, 2006. 48(1233): p. 34-5.

161. Parker, A.S., et al., Structure-guided deimmunization of therapeutic proteins. J Comput Biol, 2013. 20(2): p. 152-65.

162. Choi, Y., K.E. Griswold, and C. Bailey-Kellogg, Structure-based redesign of proteins for minimal T-cell epitope content. J Comput Chem, 2013. 34(10): p. 879-91.

163. Ziolkowska, N.E., et al., Domain-swapped structure of the potent antiviral protein griffithsin and its mode of carbohydrate binding. Structure, 2006. 14(7): p. 1127-35.

164. Ziolkowska, N.E., et al., Crystallographic, thermodynamic, and molecular modeling studies of the mode of binding of oligosaccharides to the potent antiviral protein griffithsin. Proteins, 2007. 67(3): p. 661-70.

165. Moulaei, T., et al., Monomerization of viral entry inhibitor griffithsin elucidates the relationship between multivalent binding to carbohydrates and anti-HIV activity. Structure, 2010. 18(9): p. 1104-15.

166. Ziolkowska, N.E., et al., Crystallographic studies of the complexes of antiviral protein griffithsin with glucose and $\mathrm{N}$-acetylglucosamine. Protein Sci, 2007. 16(7): p. 1485-9.

167. Xue, J., et al., The griffithsin dimer is required for high-potency inhibition of HIV-1: evidence for manipulation of the structure of gp120 as part of the griffithsin dimer mechanism. Antimicrob Agents Chemother, 2013. 57(8): p. 3976-89.

168. Giomarelli, B., et al., Recombinant production of anti-HIV protein, griffithsin, by auto-induction in a fermentor culture. Protein Expr Purif, 2006. 47(1): p. 194-202. 
169. O'Keefe, B.R., et al., Scaleable manufacture of HIV-1 entry inhibitor griffithsin and validation of its safety and efficacy as a topical microbicide component. Proc Natl Acad Sci U S A, 2009. 106(15): p. 6099-104.

170. Meuleman, P., et al., Griffithsin has antiviral activity against hepatitis $C$ virus. Antimicrobial agents and chemotherapy, 2011. 55(11): p. 5159-67.

171. Ferir, G., et al., Combinations of griffithsin with other carbohydrate-binding agents demonstrate superior activity against HIV Type 1, HIV Type 2, and selected carbohydrate-binding agent-resistant HIV Type 1 strains. AIDS Res Hum Retroviruses, 2012. 28(11): p. 1513-23.

172. O'Keefe, B.R., et al., Broad-spectrum in vitro activity and in vivo efficacy of the antiviral protein griffithsin against emerging viruses of the family Coronaviridae. Journal of virology, 2010. 84(5): p. 2511-21.

173. Ishag, H.Z., et al., Griffithsin inhibits Japanese encephalitis virus infection in vitro and in vivo. Arch Virol, 2013. 158(2): p. 349-58.

174. Montefiori, D.C., Evaluating neutralizing antibodies against HIV, SIV, and SHIV in luciferase reporter gene assays. Curr Protoc Immunol, 2005. Chapter 12: p. Unit 1211.

175. Box, G.E.P., J.S. Hunter, and W.G. Hunter, Statistics for Experimenters, ed. Wiley. 1978.

176. Student, The Probable Error of a Mean. Biometrika, 1908. 6(1): p. 25.

177. Wilcoxon, F., Individual Comparisons by Ranking Methods. Biometrics Bulletin, 1945. 1(6): p. 80-83.

178. Kagiampakis, I., et al., Potent strategy to inhibit HIV-1 by binding both gp120 and gp41. Antimicrob Agents Chemother, 2011. 55(1): p. 264-75.

179. Painter, R.G. and A. White, Effect of concanavalin A on expression of cell surface sialyltransferase activity of mouse thymocytes. Proc Natl Acad Sci U S A, 1976. 73(3): p. 837-41.

180. Smith, C.M., et al., Novel immunogenic peptides elicit systemic anaphylaxis in mice: implications for peptide vaccines. J Immunol, 2011. 187(3): p. 1201-6.

181. Gavrovic-Jankulovic, M., et al., A novel recombinantly produced banana lectin isoform is a valuable tool for glycoproteomics and a potent modulator of the proliferation response in $C D 3_{+}, C D 4_{+}$, and $C D 8_{+}$ populations of human PBMCs. Int J Biochem Cell Biol, 2008. 40(5): p. 929-41.

182. Ooi, L.S., S.S. Sun, and V.E. Ooi, Purification and characterization of a new antiviral protein from the leaves of Pandanus amaryllifolius (Pandanaceae). Int J Biochem Cell Biol, 2004. 36(8): p. 1440-6.

183. Liu, B., et al., Apoptosis-inducing effect and structural basis of Polygonatum cyrtonema lectin and chemical modification properties on its mannose-binding sites. BMB Rep, 2008. 41(5): p. 369-75.

184. Prettyman, J., Subcutaneous or intramuscular? Confronting a parenteral administration dilemma. Medsurg Nurs, 2005. 14(2): p. 93-8; quiz 99.

185. Hoorelbeke, B., et al., Actinohivin, a broadly neutralizing prokaryotic lectin, inhibits HIV-1 infection by specifically targeting high-mannose-type 
glycans on the gp120 envelope. Antimicrobial agents and chemotherapy, 2010. 54(8): p. 3287-301.

186. Balzarini, J., et al., Mutational pathways, resistance profile, and side effects of cyanovirin relative to human immunodeficiency virus type 1 strains with N-glycan deletions in their gp120 envelopes. J Virol, 2006. 80(17): p. 8411-21.

187. Smee, D.F., et al., Influenza $A$ (H1N1) virus resistance to cyanovirin-N arises naturally during adaptation to mice and by passage in cell culture in the presence of the inhibitor. Antivir Chem Chemother, 2007. 18(6): $p$. 317-27.

188. Huang, X., et al., Removal of two high-mannose N-linked glycans on gp120 renders human immunodeficiency virus 1 largely resistant to the carbohydrate-binding agent griffithsin. J Gen Virol, 2011. 92(Pt 10): p. 2367-73.

189. Michelow, I.C., et al., A novel L-ficolin/mannose-binding lectin chimeric molecule with enhanced activity against Ebola virus. J Biol Chem, 2010. 285(32): p. 24729-39.

190. Alexandre, K.B., et al., The lectins griffithsin, cyanovirin-N and scytovirin inhibit HIV-1 binding to the DC-SIGN receptor and transfer to CD4(+) cells. Virology, 2012. 423(2): p. 175-86.

191. Ferir, G., K.E. Palmer, and D. Schols, Synergistic activity profile of griffithsin in combination with tenofovir, maraviroc and enfuvirtide against HIV-1 clade C. Virology, 2011. 417(2): p. 253-8.

192. Moncla, B.J., et al., Degradation of naturally occurring and engineered antimicrobial peptides by proteases. Adv Biosci Biotechnol, 2011. 2(6): p. 404-408.

193. Macdougall, I.C., et al., Clinical pharmacokinetics of epoetin (recombinant human erythropoietin). Clin Pharmacokinet, 1991. 20(2): p. 99-113.

194. Azanza, J.R., B. Sadaba, and A. Gomez-Guiu, Monoclonal antibodies: Pharmacokinetics as a basis for new dosage regimens? J Oncol Pharm Pract, 2014.

195. Dostalek, M., et al., Pharmacokinetics, pharmacodynamics and physiologically-based pharmacokinetic modelling of monoclonal antibodies. Clin Pharmacokinet, 2013. 52(2): p. 83-124.

196. Streeck, $\mathrm{H}$., et al., Immunological and virological impact of highly active antiretroviral therapy initiated during acute HIV-1 infection. J Infect Dis, 2006. 194(6): p. 734-9.

197. Kassutto, S., et al., Longitudinal analysis of clinical markers following antiretroviral therapy initiated during acute or early HIV type 1 infection. Clin Infect Dis, 2006. 42(7): p. 1024-31.

198. Evering, T.H., et al., Absence of HIV-1 evolution in the gut-associated lymphoid tissue from patients on combination antiviral therapy initiated during primary infection. PLoS Pathog, 2012. 8(2): p. e1002506.

199. Lampton, L.M., Functional HIV cure achieved in Mississippi. J Miss State Med Assoc, 2013. 54(4): p. 94. 
200. Researchers describe first 'functional HIV cure' in an infant. J Miss State Med Assoc, 2013. 54(4): p. 111-2.

201. Yeboah, K.G., et al., In vitro and ex vivo characterization of lectin-labeled Mycobacterium tuberculosis antigen-containing microspheres for enhanced oral delivery. J Drug Target, 2014. 22(1): p. 34-47.

202. Zhang, N., et al., Lectin-modified solid lipid nanoparticles as carriers for oral administration of insulin. Int J Pharm, 2006. 327(1-2): p. 153-9.

203. Interim guidance for PrEP in MSM. CDC: Do not expand to other risk groups yet. AIDS Alert, 2011. 26(4): p. 44-5.

204. Nikolopoulos, G., et al., Antiretrovirals for HIV Exposure Prophylaxis. Curr Med Chem, 2012. 19(35): p. 5924-39.

205. van der Meer, F.J., et al., The carbohydrate-binding plant lectins and the non-peptidic antibiotic pradimicin A target the glycans of the coronavirus envelope glycoproteins. J Antimicrob Chemother, 2007. 60(4): p. 741-9.

206. Taha, H., et al., Parenteral Patent Drug S/GSK1265744 has the Potential to be an Effective Agent in Pre-Exposure Prophylaxis Against HIV Infection. Recent Pat Antiinfect Drug Discov, 2014.

207. Ford, S.L., et al., Lack of pharmacokinetic interaction between rilpivirine and integrase inhibitors dolutegravir and GSK1265744. Antimicrob Agents Chemother, 2013. 57(11): p. 5472-7.

208. Baert, L., et al., Development of a long-acting injectable formulation with nanoparticles of rilpivirine (TMC278) for HIV treatment. Eur J Pharm Biopharm, 2009. 72(3): p. 502-8.

209. Rouse, R.L., et al., Kidney injury biomarkers in hypertensive, diabetic, and nephropathy rat models treated with contrast media. Toxicol Pathol, 2013. 41(4): p. 662-80.

210. Harpur, E., et al., Biological qualification of biomarkers of chemicalinduced renal toxicity in two strains of male rat. Toxicol Sci, 2011. 122(2): p. 235-52.

211. Fantuzzi, G. and R. Faggioni, Leptin in the regulation of immunity, inflammation, and hematopoiesis. J Leukoc Biol, 2000. 68(4): p. 437-46.

212. Niesen, F.H., H. Berglund, and M. Vedadi, The use of differential scanning fluorimetry to detect ligand interactions that promote protein stability. Nat Protoc, 2007. 2(9): p. 2212-21.

213. DeSantis, K., et al., Use of differential scanning fluorimetry as a highthroughput assay to identify nuclear receptor ligands. Nucl Recept Signal, 2012. 10: p. e002.

214. Malik, K., et al., Differential scanning fluorimetry: rapid screening of formulations that promote the stability of reference preparations. J Pharm Biomed Anal, 2013. 77: p. 163-6.

215. Uniewicz, K.A., et al., Following protein-glycosaminoglycan polysaccharide interactions with differential scanning fluorimetry. Methods Mol Biol, 2012. 836: p. 171-82.

216. Layton, C.J. and H.W. Hellinga, Thermodynamic analysis of ligandinduced changes in protein thermal unfolding applied to high-throughput 
determination of ligand affinities with extrinsic fluorescent dyes.

Biochemistry, 2010. 49(51): p. 10831-41.

217. Uniewicz, K.A., et al., Differential scanning fluorimetry measurement of protein stability changes upon binding to glycosaminoglycans: a screening test for binding specificity. Anal Chem, 2010. 82(9): p. 3796-802.

218. Tian, X., et al., Identifying protein-protein interaction in Drosophila adult heads by Tandem Affinity Purification (TAP). J Vis Exp, 2013(82): p. 50968.

219. Ma, H., et al., A highly efficient multifunctional tandem affinity purification approach applicable to diverse organisms. Mol Cell Proteomics, 2012. 11(8): p. 501-11.

220. Bailey, D., et al., Identification of protein interacting partners using tandem affinity purification. J Vis Exp, 2012(60).

221. Babu, M., et al., Identification of protein complexes in Escherichia coli using sequential peptide affinity purification in combination with tandem mass spectrometry. J Vis Exp, 2012(69).

222. Shafey, D., et al., Identification of novel interacting protein partners of SMN using tandem affinity purification. J Proteome Res, 2010. 9(4): p. 1659-69.

223. Gloeckner, C.J., et al., Tandem affinity purification of protein complexes from mammalian cells by the Strep/FLAG (SF)-TAP tag. Methods Mol Biol, 2009. 564: p. 359-72.

224. Gloeckner, C.J., et al., A novel tandem affinity purification strategy for the efficient isolation and characterisation of native protein complexes. Proteomics, 2007. 7(23): p. 4228-34.

225. Ong, S.E., Unbiased identification of protein-bait interactions using biochemical enrichment and quantitative proteomics. Cold Spring Harb Protoc, 2010. 2010(3): p. pdb prot5400.

226. Kumar, A. and M. Snyder, Protein complexes take the bait. Nature, 2002. 415(6868): p. 123-4.

227. Anderson, N.L., et al., The human plasma proteome: a nonredundant list developed by combination of four separate sources. Mol Cell Proteomics, 2004. 3(4): p. 311-26.

228. Anderson, N.L. and N.G. Anderson, The human plasma proteome: history, character, and diagnostic prospects. Mol Cell Proteomics, 2002. 1(11): $\mathrm{p}$. 845-67.

229. Garbett, N.C., et al., Differential scanning calorimetry of blood plasma for clinical diagnosis and monitoring. Exp Mol Pathol, 2009. 86(3): p. 186-91.

230. Garbett, N.C., et al., Calorimetry outside the box: a new window into the plasma proteome. Biophys J, 2008. 94(4): p. 1377-83.

231. Garbett, N.C., et al., Detection of cervical cancer biomarker patterns in blood plasma and urine by differential scanning calorimetry and mass spectrometry. PLoS One, 2014. 9(1): p. e84710.

232. Zhou, M., et al., An investigation into the human serum "interactome". Electrophoresis, 2004. 25(9): p. 1289-98. 
233. Garbett, N.C., et al., Interrogation of the plasma proteome with differential scanning calorimetry. Clin Chem, 2007. 53(11): p. 2012-4.

234. Fish, D.J., et al., Statistical analysis of plasma thermograms measured by differential scanning calorimetry. Biophys Chem, 2010. 152(1-3): p. 18490.

235. Chaires, J.B., Calorimetry and thermodynamics in drug design. Annu Rev Biophys, 2008. 37: p. 135-51.

236. Mayilyan, K.R., Complement genetics, deficiencies, and disease associations. Protein Cell, 2012. 3(7): p. 487-96.

237. Skattum, L., et al., Complement deficiency states and associated infections. Mol Immunol, 2011. 48(14): p. 1643-55.

238. Botto, M., et al., Complement in human diseases: Lessons from complement deficiencies. Mol Immunol, 2009. 46(14): p. 2774-83.

239. Tolnay, M. and G.C. Tsokos, Complement receptor 2 in the regulation of the immune response. Clin Immunol Immunopathol, 1998. 88(2): p. 12332.

240. Morgan, B.P. and M.J. Walport, Complement deficiency and disease. Immunol Today, 1991. 12(9): p. 301-6.

241. Hudson-Peacock, M.J., et al., Systemic lupus erythematosus complicating complement type 2 deficiency: successful treatment with fresh frozen plasma. Br J Dermatol, 1997. 136(3): p. 388-92.

242. Venneker, G.T., et al., Incomplete functional deficiencies of the fourth (C4) and second (C2) components of complement in a patient with linear frontoparietal scleroderma and his family. Deficiencies determined by a gene not linked to human leukocyte antigen system. Exp Clin Immunogenet, 1996. 13(2): p. 104-11.

243. Eisenstein, B.I., I. Ofek, and E.H. Beachey, Interference with the mannose binding and epithelial cell adherence of Escherichia coli by sublethal concentrations of streptomycin. J Clin Invest, 1979. 63(6): p. 1219-28.

244. Ofek, I. and E.H. Beachey, Mannose binding and epithelial cell adherence of Escherichia coli. Infect Immun, 1978. 22(1): p. 247-54. 


\section{APPENDIX}

List of Abbreviations

ADA

ALB

ALKP

ALT

Amyl

ANOVA

AP

Asn

AUC

$\mathrm{Ba}$

BanLec

BCA

BGG

BSA

BUN
Anti-Drug Antibodies

Albumin

Alkaline Phosphatase

Alanine Aminotransferase

Amylase

Analysis of Variance

Affinity Purification

Asparagine

Area Under the Curve

Basophils

Banana Lectin

Bovine Gamma Globulin

Bicinchoninic Acid

Bovine Serum Albumin

Blood Urea Nitrogen 


\begin{tabular}{|c|c|}
\hline $\mathrm{Ca}$ & Calcium \\
\hline CBA & Carbohydrate Binding Agent \\
\hline $\mathrm{CBC}$ & Complete Blood Count \\
\hline CCR5 & Chemokine Receptor type 5 \\
\hline CD4 & Cluster of differentiation 4 \\
\hline Chol & Cholesterol \\
\hline Cmax & Maximum Concentration \\
\hline CMV & Cytomegalovirus \\
\hline ConA & Concanavalin A \\
\hline $\mathrm{C}_{\mathrm{p}}^{\mathrm{ex}}$ & Maximum Curve Height \\
\hline $\mathrm{C}_{\mathrm{p}}^{\mathrm{ex} 1}$ & Maximum Peak - Range 1 \\
\hline $\mathrm{C}_{\mathrm{p}}^{\mathrm{ex2}}$ & Maximum Peak - Range 2 \\
\hline Crea & Creatinine \\
\hline CV-N & Cyanovirin- $\mathrm{N}$ \\
\hline CXCR4 & Chemokine Receptor type 4 \\
\hline DC & Dendritic cell \\
\hline DC-SIGN & Dendritic Cell Specific ICAM-3 Grabbing Non-integrin \\
\hline DNA & Deoxyribonucleic acid \\
\hline DSC & Differential Scanning Calorimetry \\
\hline DSF & Differential Scanning Fluorimetry \\
\hline EDTA & Ethylenediaminetetracetic Acid \\
\hline ELISA & Enzyme Linked Immunosorbent Assay \\
\hline ENV & Envelope \\
\hline
\end{tabular}




\begin{tabular}{|c|c|}
\hline Eo & Eosinophils \\
\hline ER & Endoplasmic Reticulum \\
\hline G-CSF & Granulocyte Colony Stimulating Factor \\
\hline Glob & Globulin \\
\hline GM-SCF & Granulocyte Macrophage Colony Stimulating Factor \\
\hline gp120 & Glycoprotein 120 \\
\hline gp41 & Glycoprotein 41 \\
\hline GRFT & Griffithsin \\
\hline $\mathrm{GRO} / \mathrm{KC}$ & Growth Related Oncogene Keratinocyte Cytokine \\
\hline $\mathrm{HA}$ & Hemagglutinin \\
\hline HAART & Highly Active Anti-Retroviral Treatment \\
\hline $\mathrm{HCL}$ & Hydrochloride \\
\hline HCT & Hematocrit \\
\hline $\mathrm{HCV}$ & Hepatitis C Virus \\
\hline HGB & Hemoglobin \\
\hline HIV & Human Immunodeficiency Virus \\
\hline HPLC & High Performance Liquid Chromatography \\
\hline HSV & Herpes Simplex Virus \\
\hline $\mathrm{ID}_{50}$ & Inhibitory Dilution \\
\hline IFN & Interfereon \\
\hline $\lg$ & Immunoglobulin \\
\hline $\lg G$ & Immunoglobulin G \\
\hline IL & Interleukin \\
\hline
\end{tabular}




\begin{tabular}{|c|c|}
\hline IP & Interfereon Inducible Protein \\
\hline $\mathrm{IP}-10$ & Interfereon Inducible Protein 10 \\
\hline JEV & Japanese Encephalitis Virus \\
\hline $\mathrm{kDa}$ & Kilodaltons \\
\hline KSHV & Karposi's Sarcoma Associated herpesvirus \\
\hline Ly & Lymphocytes \\
\hline MBL & Mannose Binding Lectin \\
\hline $\mathrm{MCHC}$ & Mean Corpuscular Hematocrit Concentration \\
\hline MCP & Monocyte Chemotactic protein \\
\hline MCV & Mean Corpuscular Volume \\
\hline MIP & Macrophage Inflammatory Protein \\
\hline Mo & Monocytes \\
\hline MPV & Mean Platelet Volume \\
\hline MSM & Men who have sex with men \\
\hline PBMC & Peripheral Blood Mononuclear cell \\
\hline PBS & Phosphate Buffered Saline \\
\hline PBS-T & Phosphate Buffered Saline - Tween 20 \\
\hline PEP & Post Exposure Prophylaxis \\
\hline $\mathrm{PHA}$ & Phytohemagluttinin \\
\hline Phos & Phosphorus \\
\hline PLT & Platelet \\
\hline PrEP & Pre-Exposure Prophylaxis \\
\hline RBC & Red Blood Cells \\
\hline
\end{tabular}




$\begin{array}{ll}\text { RDW } & \text { Red Blood Cell Width } \\ \text { RNA } & \text { Ribonucleic acid } \\ \text { SARS-Cov } & \text { Sudden Acute Respiratory Syndome CoronaVirus } \\ \text { Ser } & \text { Serine } \\ \text { SVN } & \text { Scytovirin } \\ \text { TasP } & \text { Treatment as Prevention } \\ \text { Tbil } & \text { Total Bilirubin } \\ \text { TBS } & \text { Tris Buffered Saline } \\ T_{\text {fm }} & \text { First Moment Temperature } \\ \text { Thr } & \text { Threonine } \\ T_{\text {max }} & \text { Maximum Temperature } \\ \text { TMB } & \text { 3,3'5,5'-tetramethylbenzidine } \\ \text { TMV } & \text { Tobacco Mosaic Virus } \\ \text { TP } & \text { Total Protein } \\ \text { Vd } & \text { Volume of Distribution } \\ \text { VEGF } & \text { Vascular Endothelial Growth Factor } \\ \text { WBC } & \text { White Blood Cells }\end{array}$


CURRICULUM VITA

\section{BIOGRAPHICAL}

$\begin{array}{lll}\text { Name: } & \text { Christopher Lynn Barton } & \text { Birth Date: } \\ \text { Home Address: } & 206 \text { Oxford Place } & \text { Birth Place: Elgin, IL } \\ & \text { Louisville, KY } 40202 & \end{array}$

Home Phone: $\quad$ 502-418-2016

Business Address: Dept. of Pharmacology Citizenship: USA

E-Mail: Clbart01@louisville.edu

Business Phone: $\quad$ 502-852-2303

\section{EDUCATION AND TRAINING}

\section{UNDERGRADUATE}

Dates attended

Major Subject

1994-1998

Biology

\section{GRADUATE}

Dates attended

Major Subject

2009-2011
Name and location

of institution

University of Louisville

Louisville, KY
Degree received and date

Bachelor of Arts May 1998
Name and location of institution University of Louisville
Degree received and date

Master of Science 


\begin{tabular}{cll}
$\begin{array}{c}\text { Pharmacology \& } \\
\text { Toxicology }\end{array}$ & Louisville, KY & August 2011 \\
$\begin{array}{c}\text { 1999-2002 } \\
\text { Law }\end{array}$ & $\begin{array}{l}\text { University of Louisville } \\
\text { Louisville, KY }\end{array}$ & $\begin{array}{l}\text { Juris Doctor } \\
\text { May 2002 }\end{array}$ \\
$\begin{array}{c}\text { 2011-Present } \\
\text { Pharmacology \& } \\
\text { Toxicology }\end{array}$ & $\begin{array}{l}\text { University of Louisville } \\
\text { Louisville, KY }\end{array}$ & $\begin{array}{l}\text { Doctorate } \\
\text { August 2011 }\end{array}$ \\
\hline
\end{tabular}

PROFESSIONAL EXPERIENCE

\begin{tabular}{|c|c|c|}
\hline Years & Name and Location & Title \\
\hline 2013-present & $\begin{array}{l}\text { University of Louisville } \\
\text { Louisville, KY }\end{array}$ & $\begin{array}{l}\text { Technology } \\
\text { Transfer Manager }\end{array}$ \\
\hline 2008-2009 & $\begin{array}{l}\text { Barton Law Office, PLLC } \\
\text { Louisville, KY }\end{array}$ & Attorney \\
\hline $2005-2008$ & $\begin{array}{l}\text { Kentucky Office of Insurance } \\
\text { Frankfort, KY }\end{array}$ & $\begin{array}{l}\text { Attorney/Division } \\
\text { Director }\end{array}$ \\
\hline 2004-2005 & $\begin{array}{l}\text { Barton Law Office, PLLC } \\
\text { Louisville, KY }\end{array}$ & Attorney \\
\hline 2003-2004 & $\begin{array}{l}\text { CNAC } \\
\text { Louisville, KY }\end{array}$ & In-House Counsel \\
\hline
\end{tabular}

MEMBERSHIPS in PROFESSIONAL and SCIENTIFIC SOCIETIES

Organization

Year

Association of University Technology Managers 2013-present

Kentucky Bar Association 2002-present

United States Patent and Trademark Office 2002-present 